\title{
Hot Nuclear Matter
}

\author{
Scott Chapman \\ Ph.D. Thesis \\ PHYSICS DEPARTMENT \\ University of California \\ and \\ NUCLEAR SCIENCE DIVISION ${ }^{\dagger}$ \\ Lawrence Berkeley Laboratory \\ University of California \\ Berkeley, CA 94720
}

November, $199^{\circ} \mathrm{C}$ 


\section{Contents}

$\begin{array}{ll}\text { Introduction } & 1\end{array}$

1 Nuclear Stopping Power 6

1.1 Nuclear Transparency in $15 \mathrm{AGeV} \mathrm{Si}+\mathrm{Au}$ Reactions? . . . . . . . . ?

1.2 Nuclear Stopping Power at $15 \mathrm{GeV} /$ nucleon . . . . . . . . . . 16

1.2 .1 Introduction . . . . . . . . . . . . . 16

1.2.2 The Hadronic Fireball . . . . . . . . . . . . 17

1.2.3 The Firestreak and String Models . . . . . . . . . . . 23

1.2.4 Model Independent Fits . . . . . . . . . . . 26

1.2 .5 Hybrid Models ................... 36

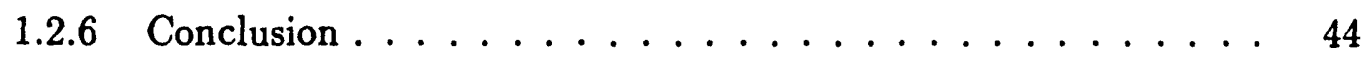

1.2 .7 Appendix ....................... 45

1.3 Talk from Quark Matter ' $91 \ldots \ldots$. . . . . . . . . . 47

1.3.1 Introduction ............................ 47

1.3.2 Comparison of Models to the Data . . . . . . . . . . 48

1.3.3 Model Independent Fits ................ 51

1.3.4 Conclusion ......................... 54

1.4 Recent Developments . . . . . . . . . . . . . . 55

2 Effective Action for SU(N) at Finite Temperature 62

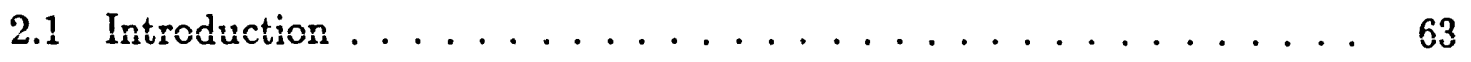

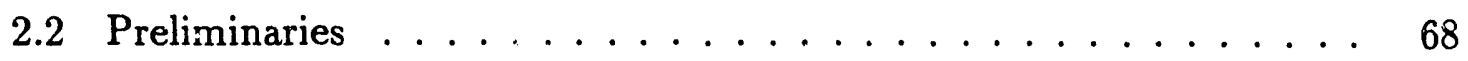


$2.2 .1 \quad$ Zero Modes . . . . . . . . . . . . . . . . . . . . . 69

2.3 Covariant Derivative Expansion . . . . . . . . . . . . 72

2.3.1 Ghosts . . . . . . . . . . . . . . . . . . . . . 74

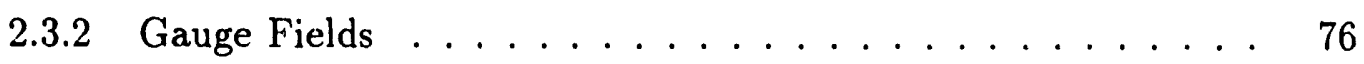

2.3.3 Renormalization. . . . . . . . . . . . . . . . . . 77

2.3.4 Constant Background $A_{0}$ Field $\ldots \ldots \ldots \ldots \ldots$

2.4 Application to Dyons . . . . . . . . . . . . . . . . . . 80

2.5 Improved Expansion $\ldots \ldots \ldots \ldots \ldots \ldots \ldots$

2.6 More Dyons . . . . . . . . . . . . . . . . . . 88

2.7 Monopoles . . . . . . . . . . . . . . . . . . . . . 90

2.7 .1 Negative Modes . . . . . . . . . . . . . . . . . 92

2.7.2 Generalization to SU(3) with fermions . . . . . . . . 95

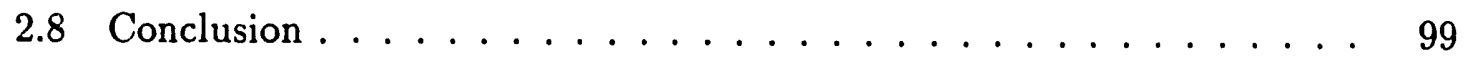

A Integrals, Sums and Functionals . . . . . . . . . . . . . 100

B Effective Potentials for $T \neq 0 \mathrm{SU}(\mathrm{N}) \ldots \ldots \ldots \ldots \ldots$

C Allowed Gauge Transformations . . . . . . . . . . . . . . . . . 104

$\begin{array}{ll}\text { Bibliography } & 106\end{array}$ 


\section{List of Figures}

A Phase Diagram for QCD $\ldots \ldots \ldots \ldots \ldots \ldots$

1.1.1 Fireball, Firestreak and Lund Compared to Central Si+Au Data . 8

1.1.2 Model Independent Fits to Central Si+Au Data . . . . . . . . . 11

1.1.3 Kaon Data . . . . . . . . . . . . . . . . . . . . . . 12

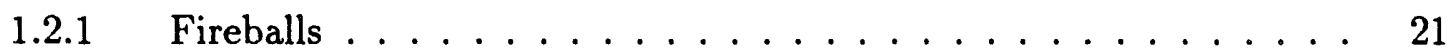

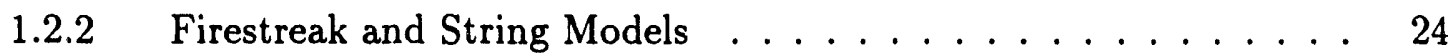

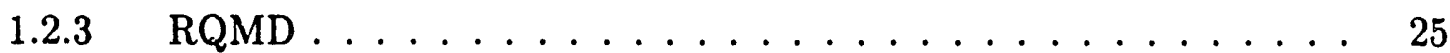

1.2.4 Fits to E802 Spectrometer Protons . . . . . . . . . . . 27

1.2.5 Fits to E802 Spectrometer Pions . . . . . . . . . . . . . 28

1.2.6 Fits to E802 Spectrometer Kaons and $P_{\perp} \ldots \ldots \ldots \ldots \ldots$

1.2.7 Pseudorapidity Data of Charged Particles . . . . . . . . . 32

1.2.8 E814 Leading Neutrons . . . . . . . . . . . . . . . . . . 33

1.2.9 Predictions for the Particle Calorimeter . . . . . . . . . . . . . 34

1.2.10 Phase Space Restrictions of One Stopping Scenario . . . . . . . 37

1.2.11 Double Firestreaks . . . . . . . . . . . . . . . . . . . . 39

1.2.12 Multicomponent Model Fits . . . . . . . . . . . . . . 40

1.2.13 Multicomponent Model Fits for Kaons . . . . . . . . . . . . 41

1.2.14 Predictions for Central $\mathrm{Au}+\mathrm{Au} \ldots \ldots \ldots \ldots$

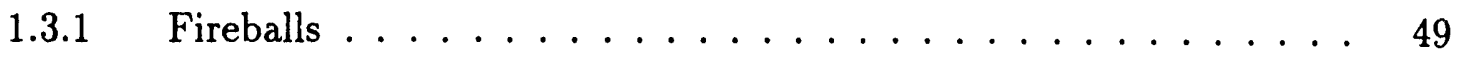

1.3.2 Firestreak and String Models . . . . . . . . . . . . . . 50

1.3.3 Momentum Distribution of Model Independent Fits . . . . . . . 52 
1.3.4 Pseudorapidity Data of Charged Particles . . . . . . . . 53

$1.4 .1 \quad P_{\perp}$ Fits vs. $M_{\perp}$ Fits $\ldots \ldots \ldots \ldots \ldots \ldots \ldots$

$1.4 .2 \quad$ New vs. Old Data for $\mathrm{Si}+\mathrm{Au} \ldots \ldots \ldots \ldots \ldots$

1.4.3 Comparison of Predictions to Preliminary Au+Au Data . . . . . 59

2.3.1 SU(2) Effective Potential . . . . . . . . . . . . . 79

$2.7 .1 \mathrm{SU}(3)$ Effective Potential . . . . . . . . . . . . . 96

2.7.2 Contour Plot of Pure Gauge SU(3) $\ldots \ldots \ldots \ldots \ldots$

2.7.3 Contour Plot of $\mathrm{SU}(3)$ with 2 Fermions $\ldots \ldots \ldots \ldots$ 


\section{Acknowledgements}

I am grateful to Matt Bloomer, Shoji Nagamiya, Flemming Videbaek, Sam Lindenbaum, Johanna Stachel, Jimmy Dee and Chuck Parsons for extensive discussions regarding the AGS data used in chapter one. Chapter two was originally inspired by the enlightening ideas and marathon seminars of Janos Polonyi and Michael Oleszczuk. I would also like to thank my campus advisor Mahiko Suzuki as well as Korkut Bardacki and Mary K. Gaillard for valuable discussions about effective actions, magnetic monopoles and dynamical stability. I greatly appreciate both Stanley Mandelstam and Joseph Cerny for taking their time to wade through this thesis. In addition, I am grateful for the interest, suggestions and moral support of the Nuclear Theory group at LBL. Finally, I would like to thank my advisor Miklos Gyulassy, whose ardent desire to find and understand truth has continually challenged and guided me in my research.

This work was supported by the Director, Office of Energy Research, Division of Nuclear Physics of the Office of High Energy and Nuclear Physics of the U.S. Department of Energy under Contract No. DE-AC03-76SF00098. 


\section{Introduction}

It is now generally believed that quarks (and antiquarks) are the fundamental building blocks of all nuclear matter. Unlike electrons and other fundamental particles, however, quarks have never been detected in isolation. Only composite particles called hadrons, which are bound state combinations of quarks and antiquarks, have been observed experimentally. The reason for this apparent confinement of quarks into hadrons is a mystery which must ultimately be solved by any theory purporting to describe the strong nuclear force. Currently, the best candidate for this theory, quantum chromodynamics (QCD), attributes a "color" to each quark and antiquark in such a way that each hadron corresponds to a "colorless" combination held together by particles called gluons. Although this idea is useful in explaining some of the symmetries seen in the various species of hadronic particles, it is still not known why only colorless combinations have been observed experimentally. Many believe that the answer to this puzzle may lie somewhere in the depths of QCD, but the theory is so complex that no one has yet been able to discover it.

To obtain a better understanding of QCD, it is therefore necessary to simplify the theory by making reasonable approximations. Since gluonic interactions become weaker at higher energies, the simplest approximation for high energy processes can be obtained by treating quarks and gluons as free non-interacting particles. Better approximations can then be arrived at by calculating perturbative corrections which take into account more and more complicated interactions. Perturbative QCD formulated in this way has been remarkably successful in describing the results of high energy experiments like electron-positron annihilation and jet production[LLew]. Another commonly used approximation is to treat spacetime as a four dimensional lattice of separated points (rather than a continuous volume) and to perform calculations only at these points. Presumably, calculating on a lattice with an infinite number of points infinitely close together would be the same as performing a continuous calculation and so would provide exact answers to QCD. Due to the limitations of present 
day computers, however, months are required to obtain results from a lattice of only $16^{4}$ points[Christ]. Nevertheless, making lattice calculations is currently the only way that QCD can be tested against observed low energy phenomena like the rest masses of hadrons. For example, the ratio of the proton mass to the $\rho$ meson mass is measured to be 1.2, whereas lattice QCD obtains a value of 1.5 [Fuku]. On the basis of this and other comparisons, many people argue that lattice QCD calculations should be accurate to within $20-30 \%$.

One of the most intriguing predictions of lattice QCD is that there should be a phase transition into a new form of matter when the temperature is increased to approximately $150-200 \mathrm{MeV}$. In this new phase, commonly called the quark-gluon plasma (QGP), highly energetic quarks and gluons would no longer be bound inside of colorless hadrons, so it would seem that perturbative techniques should be applicable. High temperature perturbation theories have been developed[Kap79] and refined[Bra90], but subtle problems in the theories lead to a complete breakdown of the perturbation expansion when sufficiently complex interactions are considered[Lin80]. These problems could be a clue that non-perturbative objects like color magnetic monopoles must be considered in order to obtain an accurate picture of QCD at high temperature. It is also conceivable that the lessons learned in calculating the properties of hot QCD systems could shed light on the tantalizing question of quark confinement in hadrons at low energies.

For these reasons, it is of interest to see whether a quark-gluon plasma can be created in the laboratory. Consequently, there are a number of relativistic heavy ion experiments currently being run or planned whose main aim is to create and observe the properties of a QGP. For example at Brookhaven's Alternating Gradient Synchrotron (AGS), silicon projectile nuclei have been accelerated to momenta of 14.6 $\mathrm{GeV} / \mathrm{c}$ per nucleon (0.998 times the speed of light) before colliding with gold targets at rest[E802]. The hope is that when a silicon nucleus and a gold nucleus collide head on at these energies, they will stick together in a highly compressed state for a short time, rather than immediately pass through one another. If such nuclear stopping does in fact occur, then it is possible that a super-dense lump of hot nuclear inatter 
(5-8 times normal nuclear density) can be created in these reactions. Furthermore, if the matter is hot and dense enough, then the phase transition could occur from hadronic matter to a quark-gluon plasma. This plasma would subsequently expand and cool until finally "freezing" back into hadrons which could then be detected by the experiment (fig. A). By examining in detail the final hadron distributions, it is hoped that one would be able to discern whether or not a QGP had been formed in the collision, and possibly even be able to determine some of its properties.

Before searching for signs of a QGP, however, it is important to determine whether or not the colliding nuclei were able to stop one another in the first place. The first chapter of my thesis is comprised of three published papers and a summary of recent

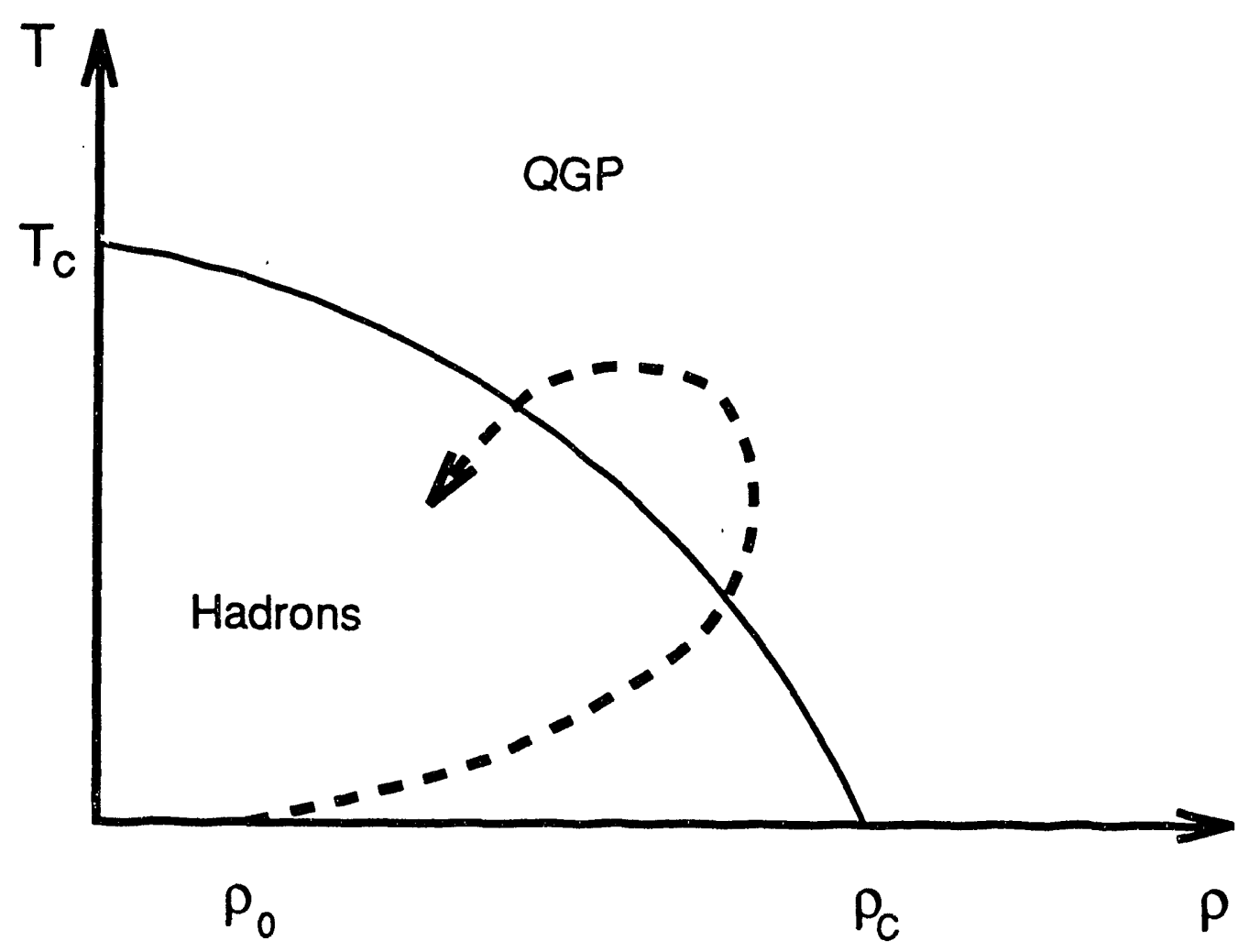

Figure A. The phase diagram for QCD. Temperature is plotted on the vertical axis, and nuclear matter density is plotted on the horizontal axis with normal nuclear density denoted by $\rho_{0}$. The solid arc is a schematic representation of the phase transition from hadronic matter to a quark-gluon plasma. The critical temperature $T_{c}$ is thought to be about $150-200 \mathrm{MeV}$, while the critical density $\rho_{c}$ is thought to be about 5-8 times $\rho_{0}$. Time evolution of the nuclear matter in a heavy ion collision is shown by the dotted curve. This matter compresses and heats up until a QGP is formed, then it expands, cools, and eventually "freezes" back into hadrons. 
developments which are devoted to exploring this issue. In the first paper, I showed that none of the standard event generators could reproduce the silicon on gold data reporied by the E802 collaboration at the AGS[E802]. Furthermore, I showed that if these data are correct, then the nuclei involved exhibit an unusually high degree of transparency to each other. This was a very unpopular and surprising result, since I had shown that the amount of stopping implied by the data was even less than that observed for proton-proton collisions at similar energies, making the possibility of QGP formation at the AGS appear to be impossibly remote. In the second paper, I pointed out discrepancies between various data sets from different experimental groups at the AGS, explored alternative scenarios in which I assumed that the normalization of E802 data was off by more than $30 \%$, and made predictions for the results of upcoming gold on gold collision experiments. The last paper is the published version of a talk given at the Quark Matter '91 conference in which I showed that if key points of the E802 data were off by $40-70 \%$, then scenarios could be found in which the expected amount of nuclear stopping was achieved and agreement could be obtained between E802, E810 and E814 data sets. Although no new data has yet been officially published by the E802 (E866) collaboration, a recent $\mathrm{Ph} . \mathrm{D}$. thesis from an E802 collaborator reports that new measurements and newly found systematic errors have had the effect of increasing the normalization of the E802 data by $10-20 \%$ for some points and as much as $50-100 \%$ for others[Pars]. In my summary of recent developments, I show that the reported increases are helpful, but that more corrections are needed for some of the data points in order to establish overall agreement with the other experiments and theoretical expectations. In addition, I compare my gold on gold predictions with new preliminary data by E866[E866] and show that the amount of stopping occurring in these reactions is still inconclusive. Consequently, many more measurements will have to be made before it will be possible to prove (or disprove) that the amount of nuclear stopping required for QGP formation is actually being realized in these reactions. 
The second chapter of my thesis deals with some of the more formal mathematical aspects of QCD and similar theories at high temperatures. Since there are fundamental problems with the normal perturbative expansions of these theories[Lin80], non-perturbative techniques must be explored. For example, rather than treat the QCD vacuum as a state without any background fields, nontrivial background field configurations can be used to model the vacuum. In perturbative calculations, the quarks and gluons of the QGP arise from considering quantum fluctuations of the fields around the trivial vacuum. In non-perturbative semi-classical calculations, on the other hand, quantum fluctuations around complicated background fields must be considered. These calculations are far more complex than their perturbative counterparts, and exact solutions are only known for some very special cases[tHo76]. In order to consider other more general cases, I have developed an approximation technique in which the quantum solutions are expressed in terms of a covariant derivative expansion. Similar expansions have been made for theories at zero temperature[Che87], but this is the first such calculation for QCD and $S U(N)$ theories at finite temperature. As a first application of this technique, I show that certain color magnetic monopole background configurations are unstable to quantum fluctuations and that a background gas of dyons and antidyons would only be able to stabilize if the gas was so dense that the dyons were overlapping. These results cast doubt on previous speculations that a simple monopole plasma could regulate the infrared magnetic singularities which plague hot QCD. While we rule out two types of magnetically charged configurations, the development in this thesis of the covariant derivative expansion should prove useful in future studies of other possible background configurations, not only in hot QCD, but more generally in hot gauge theories.

The goal in this thesis is thus twofold: The first is to investigate the feasibility of using heavy ion collisions to create conditions in the laboratory which are ripe for the formation of a quark-gluon plasma. The second is to develop a technique for studying some of the many non-perturbative features of this novel phase of matter. 
Chapter 1

Nuclear Stopping Power 


\title{
1.1 Nuclear Transparency in 15 AGeV Si+Au Reactions?
}

\author{
by Scott Chapman and Miklos Gyulassy
}

(Published in Physical Review Letters 67 (1991) p. 1210)

\begin{abstract}
Recent data on central $\mathrm{Si}+\mathrm{Au}$ collisions at $15 \mathrm{AGeV}$ are shown to imply an unexpected high degree of nuclear transparency. The paucity of observed midrapidity protons and pions suggests that up to one half of the projectile nucleons may lose less than one unit of rapidity after traversing 5-10 fm of nuclear matter.
\end{abstract}

The first detailed spectra of $p, \pi^{ \pm}$, and $K^{ \pm}$from central $S i+A u$ reactions 14.6 $\mathrm{AGeV} / \mathrm{c}$ have been reported recently by the E802 coliaboration[E802] at the AGS. These data are of interest in connection with estimating the nuclear stopping power and assessing whether high baryon density matter can be produced in nuclear collisions. Previous indirect data on transverse energy spectra and leading baryon spectra have been interpreted[E814, Stach] as evidence for a large amount of nuclear stopping in such reactions. However, in Ref.[Gyu90] we noted that the paucity of pions and the shape of the proton rapidity distribution measured by E802[E802] were more indicative of nuclear transparency at least for light ion induced reactions. Our aim in this letter is to analyze the new data in detail and to estimate the nuclear stopping power in this reaction using a multicomponent firestreak model.

The data that we focus on are shown in Figs. 1.1.1-1.1.3. The $p, \pi^{-}, K^{ \pm}$rapidity densities in central $\mathrm{Si}+\mathrm{Au}$ collisions are shown in the upper panels. The lower panels show the transverse momentum slope parameter, $T(y)$, obtained by fitting the invariant distributions at each rapidity with $\exp \left(-m_{\perp} / T(y)\right)$. The curves and histograms show the results based on the models discussed below. Also shown are extrapolations of the E814 leading neutron data[E814] from a 0.8 degree cone assuming the above $m_{\perp}$ distribution with $T$ varied between 0.1 to $0.2 \mathrm{GeV}$ for their $E_{T}>13 \mathrm{GeV}$ trigger. 


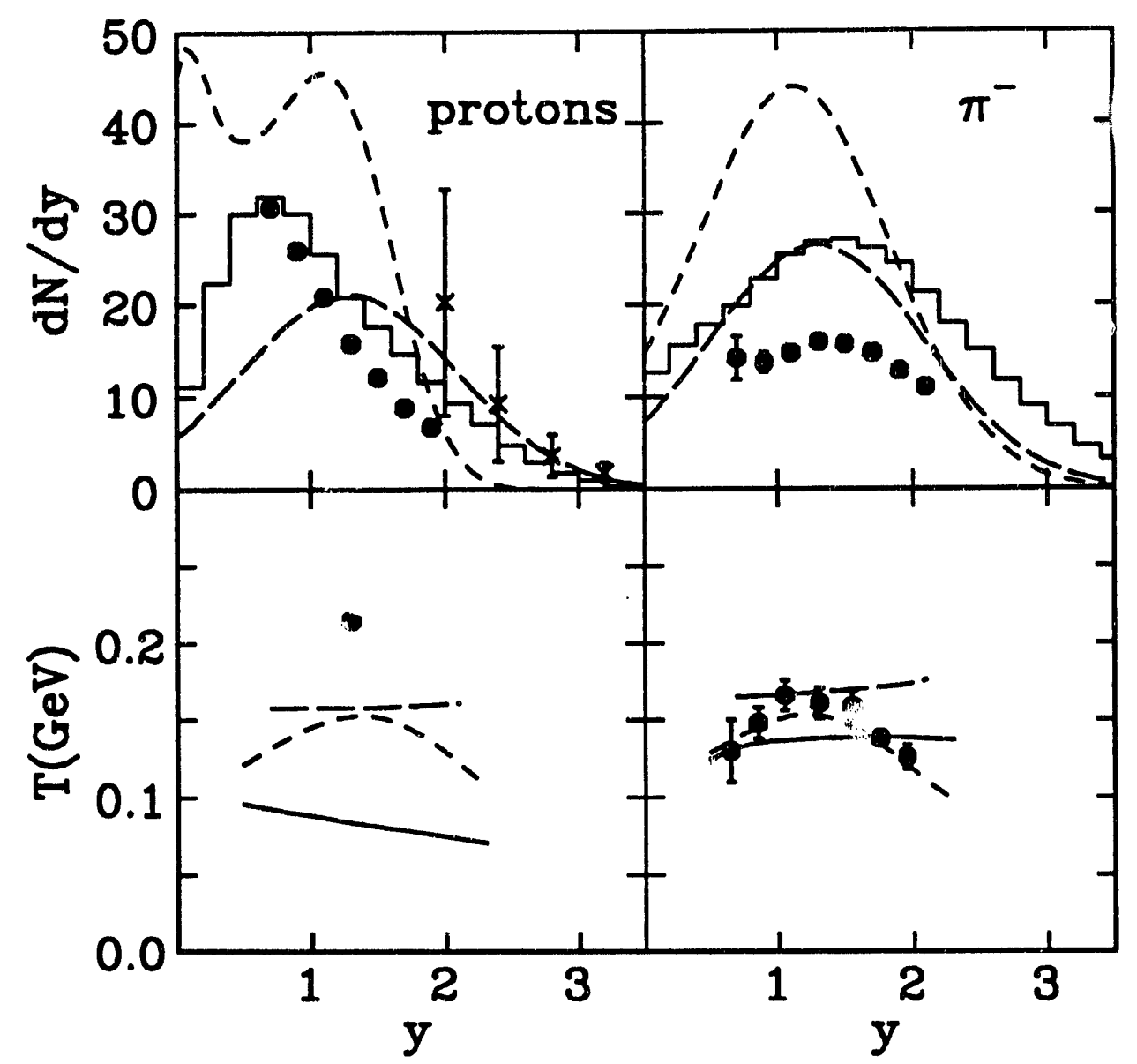

Figure 1.1.1: The proton and $\pi^{-}$rapidity distributions and transverse mass slope parameters in central $S i+A u$ reactions[E802] (solid dots). Short dashed curves and histograms show results from the firestreak[Gosset, Myers] and Lund models[Gyu 87], resp.. The long dashed curves show results form the Landau hydrodynamic model[Stach]. The extrapolated leading neutron data[E814] are indicated by the crosses together with estimated extrapolation uncertainties.

Based on $p+A \rightarrow p+X$ data at energies $E_{\text {lab }} \gtrsim 100 \mathrm{GeV}[\mathrm{pA}]$, it was expected that in central $S i+A u$ reactions the average rapidity of projectile baryons would be shifted downward by $\Delta y \sim 2.5$ while the rapidity of participant target baryons should be shifted upward by $\Delta y \gtrsim 1$. Therefore a substantial amount of equilibration between projectile and target baryons was expected to occur at $15 \mathrm{AGeV}$ where the total rapidity gap is only 3.5 . We tinerefore compare the data first with the firestreak model[Gosset, Myers]. The short dashed curves in Figs. 1.1.1 and 1.1.3 show the results obtained with a cut on impact parameters $b \leq 2.9 \mathrm{fm}$. The severe discrepancy 
besween the data and the calculated results is obvious. No reasonable variation of the freeze-out density was found to improve this situation. Also shown by the long dashed curves in fig. 1.1.1, are the results using the Landau hydrodynamic fireball of Ref.[Stach]. While the proton distribution is in agreement with the extrapolated E814 data, it fails to account for the ramp form of E802 proton data, the difference between the pion and pioton slope parameters, and the absolute pion yield. In Ref.[Brown, BrownEr] a hydrochemical version of the fireball model was able to reproduce the pion and kaon spectra, but that model also failed to account for the form and magnitude of the observed proton distribution. It follows that if the E802 data are correct all such equilibrium models assuming complete nuclear stopping are ruled out by the absence of a peak of $d N_{p} / d y$ near $y \sim 1.2$, the small value of $d N_{p} / d y \approx 7$ at $y \sim 2$, and the small number of $\pi^{-}$observed at mid rapidity.

We therefore consider next non-equilibrium dynamical models such as the multistring Lund Fritiof Model[Lund]. In that model multiple interactions are assumed to excite baryon strings which fragment independently and without final state interactions. Such phenomenological string models have been successful in accounting for many of the features of multiparticle production in $p+A$ and $B+A$ collisions at higher energies $E_{l a b}>60 \mathrm{AGeV}[\mathrm{QM} 88]$. The histograms in fig. 1.1.1 show the results from the ATTILA version[Gyu87] of the Fritiof model for this reaction for the same range of impact parameters. While the ramp form of the proton distribution is much better reproduced, the proton slopes are much smaller than observed. In addition, the $\pi^{-}$rapidity density is overpredicted by $70 \%$. We note that RQMD string '- model[RQMD91, RQMDpi] also overpredicts the pion rapidity density by $70 \%$.

Having seen that the above simple equilibrium and nonequilibrium models for nuclear collision dynamics fail to reproduce the new data, we consider next a model independent fit in order to isolate possible causes for the discrepancies. In particular, this fit allows us to take into account all of the observed energy in longitudinal and transverse motion, pion production and kaon production. The measured transverse 
momentum distributions were fit with a form

$$
d N_{i} / d y d^{2} p_{\perp}=\rho_{i}(y) \exp \left(-m_{i \perp} / T_{i}(y)\right)
$$

where the slope parameters, $T_{i}(y)$, were parameterized by sums of Gaussians in rapidity. The data reported in [E802] together with unpublished data from [B̈loo] were used to fix these slopes. The pion and kaon rapidity distributions were parameterized in terms of independent Gaussians. For the unobserved neutral mesons we assumed $\pi^{0}=\left(\pi^{+}+\pi^{-}\right) / 2, K^{0}=K^{+}$, and $\bar{K}^{0}=K^{-}$. The nucleon rapidity distribution was taken to be parabolic in the region $0<y<3.0$ with a linearly dropping tail from $3.0<y<3.5$ and Gaussian tail $y<0$. In the high rapidity region we allowed for an extra Gaussian distribution of baryons to test for nuclear transparency. For neutrons we assumed that $\rho_{n}(y)=132 / 93 \rho_{p}(y)$ to be on the safe side (i.e., allowing for larger unobserved neutral baryon energy than expected in the projectile fragmentation region). Total baryon conservation was enforced.

We found that without an extra, high rapidity, baryon contribution the total longitudinal momentum carried by nucleons and mesons integrated over all of phase space was $165 \mathrm{GeV} / \mathrm{c}$ less than the total initial momenturn $\left(P_{0}=409 \mathrm{GeV} / \mathrm{c}\right)$. To take into account possible systematic errors introduced by extrapolations to unmeasured low $p_{\perp}$ regions and depletion of the proton yield due to composite fragment formation[E802], we tried a fit to data enhanced by a factor 1.3 However, even with that enhancement the fit failed to account for $93 \mathrm{GeV} / \mathrm{c}$ of the incident longitudinal momentum!

Only by introducing an extra, high rapidity baryon contribution centered at $y=$ 2.75 with an rms width $\Delta y=0.25$ and containing approximately 11 of the 28 incident baryons were we finally able to account for all the incident momentum (and energy) to an accuracy of better than $1 \mathrm{GeV}$. This final fit is shown by the dot-dashed curves in figs. 1.1.2 and 1.1.3. We have checked that neither the $E_{T}$ nor the forward calorimeter data are sensitive to this unexpected baryon contribution in the region $2<y<3$. We emphasize that the energy contained in the observed transverse flow of baryons 
as well as in enhanced kaon production is taken into account by this fit. In addition our fit is conservative since we assumed that all the E802 rapidity densities must be multiplied by 1.3 due to systematic errors. From this analysis we conclude that the E802 spectrometer data are consistent with longitudinal momentum and baryon conservation only if a significant fraction of the projectile nucleons suffer less than one unit of rapidity shift after traversing $5-10 \mathrm{fm}$ of nuclear matter.

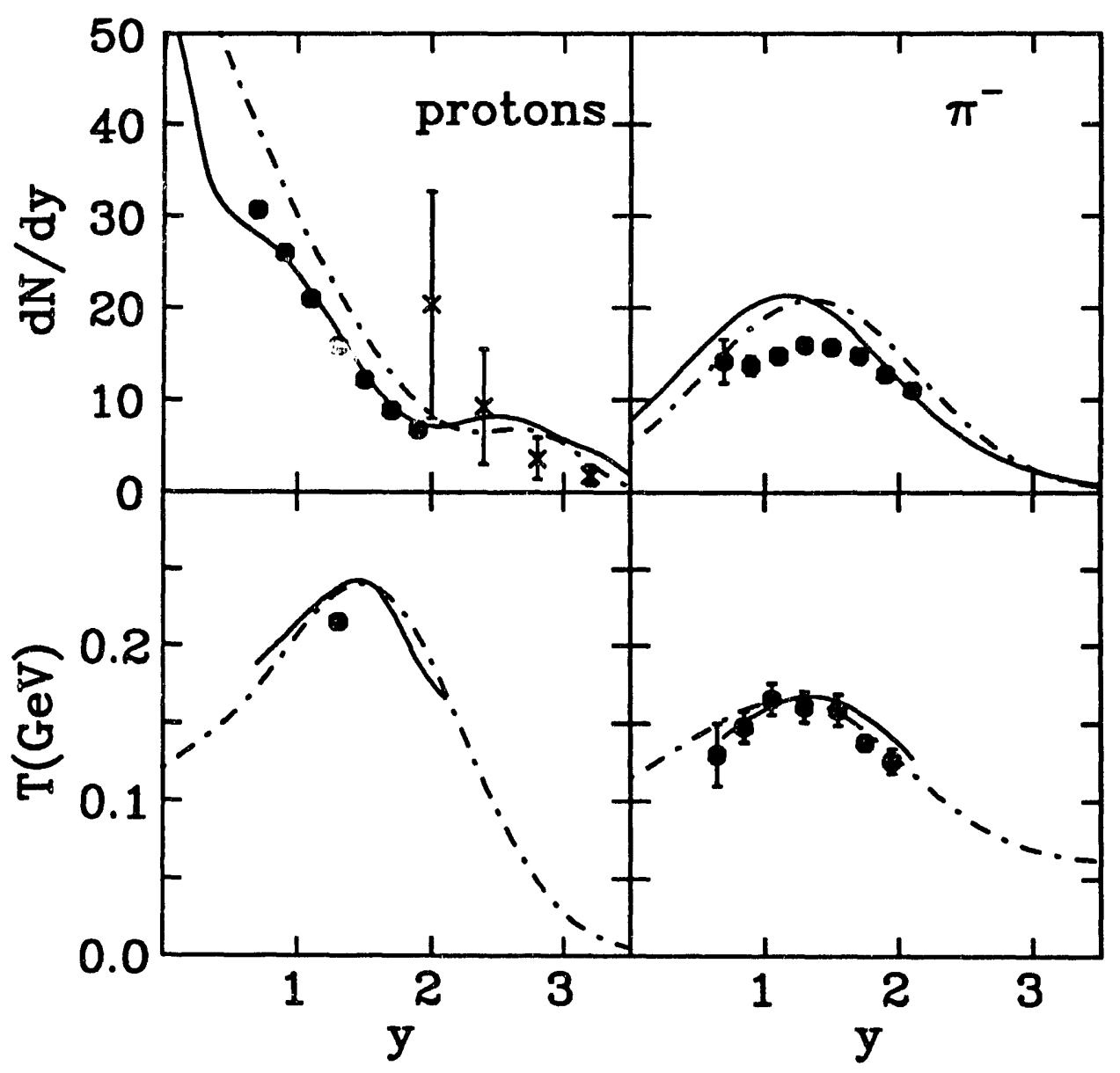

Figure 1.1.2: As in fig. 1.1.1 but compared to a constrained fit (dot-dashed) to data enhanced by a factor 1.3. The solid curves show results of our multicomponent model with $L_{s}=L_{s}^{\prime}=26 \mathrm{fm}$.

Of course, it is possible that the "central" multiplicity-triggered data actually suffered some contamination from peripheral events. Large numbers of projectile spectators from these events would then be able to account for the missing longitudi- 
nal momentum. However, unpublished data correlating the multiplicity trigger with the zero degree calorimeter (ZDC)[Bloo] suggest that the published central events had negligible numbers of projectile spectators. Furthermore, unpublished central ZDC-triggered data[Bloo] agree to within $10 \%$ with the multiplicity-triggered data considered here.

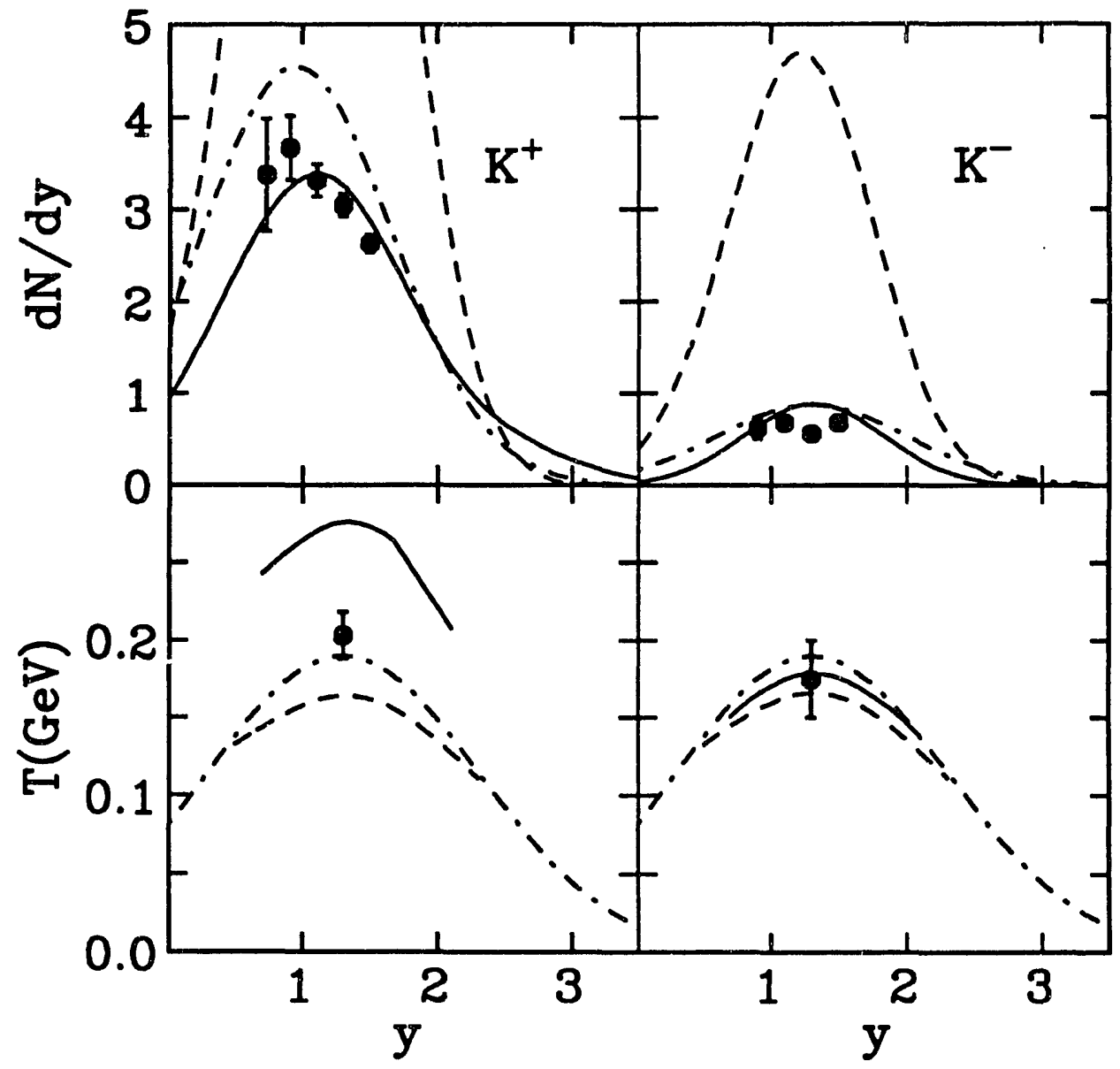

Figure 1.1.3 $\mathrm{K}^{ \pm}$data[E802] compared to firestreak (dashed), constrained fit (dotdashed), and multicomponent model (solid) calculations.

To estimate more quantitatively the nuclear stopping power implied by these data and to enable us to calculate the $A$ and impact parameter dependences of the spectra, we developed a multicomponent firestreak model with enough flexibility to deal with many complex nonequilibrium features exhibited by the $p, \pi$, and $K$ data. Instead of forming one fireball(streak) in each collision between rows of nucleons as in the 
conventional firestreak model, we allow each row-row collision to form up to four fireballs with different rapidities depending on the nuclear thicknesses involved. We found that four fireballs was the minimum necessary to reproduce all the features of the present data. While differing in detail, this model is similar to previous multicomponent fireball and hydrodynamic models[Dan, Clare]) which were introduced to take into account nuclear transparency.

In our model we assume that in a collision of two tubes of nuclear matter of transverse area $\sigma_{i n}=30 \mathrm{mb}$ containing $N_{p}$ and $N_{t}$ nucleons, the total center of mass momentum $P^{*}$ of both tubes is reduced by an amount proportional to the number of binary collisions, $N_{p} N_{t}$ :

$$
\Delta P^{*}=\delta p_{z} N_{p} N_{t}
$$

Here $\delta p_{z}$ is the average longitudinal momentum loss per inelastic collision. Defining the effective nuclear thickness, $z_{i}$, via $N_{i}=\sigma_{i n} \rho_{0} z_{i}$, the momentum shift per baryon of the projectile (target) is thus assumed to increase linearly with the target (projectile) thickness. A measure of the nuclear stopping power is given by the stopping length

$$
L_{s}=m_{N} \sinh \left(\left(y_{p}-y_{t}\right) / 2\right) /\left(\sigma_{i n} \rho_{0} \delta p_{z}\right),
$$

where $y_{p}\left(y_{t}\right)$ is the rapidity of the projectile (target) tube. For symmetric collisions with $z_{p}=z_{t}=z$, the fractional momentum loss, $\Delta P^{*} / P^{*}=z / L_{s}$, increases linearly and reaches unity when $z=L_{s}$.

We found, however, that the above two fireball model of stopping could not reproduce the apparent peaking of $T_{p}(y)$ near $y \sim 1.5$ as indicated by preliminary E802 data[Bloo]. We therefore allowed a fraction, $f_{s}$, of the baryons from both the projectile and target nucleon in each tube to stop completely in the tube-tube $\mathrm{cm}$ frame. This fraction was also assumed to increase with nuclear thickness as

$$
f_{s}=\left(z_{p} z_{t}\right)^{1 / 2} / L_{s}^{\prime}
$$

Incomplete nuclear stopping is thus modelled by three separate baryonic fireballs (for 
each row-row collision) with rapidity and baryon number controlled by two stopping lengths, $L_{s}$ and $L_{s}^{\prime}$.

The baryon transverse momentum distribution is controlled by the excitation energy per baryon, $M^{*}$, in each of these fireballs. In order to fit the preliminary $T_{p}(y)$ data[Bloo], we enforce the constraints $M^{*} \leq M_{1}^{*}=1.4 \mathrm{GeV}$ for the noncentral fireballs and $M_{c}^{*} \leq M_{2}^{*}=1.85 \mathrm{GeV}$ for the central ones. Any excess energy is assumed to be taken up by a fourth central fireball with zero baryon content (the "meson" fireball). The baryon fireball freeze-out densities are all chosen to be $\rho_{f}=\rho_{0}=0.15 \mathrm{fm}^{-3}$, while the meson fireball freeze-out temperature is chosen to be $160 \mathrm{MeV}$. In addition, to account for incomplete chemical equilibration of strange hadrons sec $n$ from fig. 1.1.3, we reduced the thermal contributions of all strange hadrons by a factor $1 / 4$. These hybrid aspects of the model essentially mimic effects in the hydrochemical model[Brown, BrownEr] without the constraint of full nuclear stopping.

The solid lines in Figs. 1.1.2, 1.1.3 show the results of this multicomponent model for $L_{s}=L_{s}^{\prime}=26 \mathrm{fm}\left(\delta p_{z}=0.22 \mathrm{GeV} / \mathrm{c}\right)$. With these parameters we recover essentially the results of the (dash-dot) fit discussed earlier. In particular, this model also leads to a high rapidity projectile contribution centered around $y \approx 2.5$ as required by energy-momentum and baryon conservation. The rather large values of these stopping lengths are surprising in view previous expectations based on $p+A$ at higher energies [pA, Mish]. Also with $L_{s}=26 \mathrm{fm}$, the fraction of projectile baryons in the central fireball is only $f_{s} \sim 1 / 3$ for $S i+A u$. This value is much less than deduced in [E814, Stach] based on transverse energy and leading neutron data and unpublished high multiplicity selected E802 $d N_{\text {ch }} / d \eta$ data.

We comment finally on the difference between collective longitudinal hydrodynamic flow and nuclear transparency. In Ref.[Stach] it was suggested that Landau hydrodynamics could account for the nonisotropic angular distributions in the $\mathrm{cm}$ frame. However, the comparison between that model and the data in fig. 1.1.1 shows that no single expanding source can account for the different maxima and 
shapes of those distributions. On the other hand, detailed one fluici hydrodynamic calculations[Stau89, Stau90] predict a nonsymmetric baryon distribution with a shoulder between $2 \lesssim y \lesssim 3$. In fact so much longitudinal collective baryon flow was predicted that the calculated pion yield falls significantly below the E802 data. It would be interesting to check if variations of the equation of state and the freeze-out condition could improve the agreement with data for this reaction. In principle only the $A$ dependence of the particle spectra can differentiate between such novel nuclear shock effects from transparency. For example, one fluid hydrodynamics predicts[Strot] a sharp peak at mid rapidity for the proton distribution in central $S i+A l$, whereas our model predicts a minimum in that case.

We conclude that none of the present models which assume complete nuclear stopping and none of the nonequilibrium string models are consistent with the new E802 data. If the normalization error of the new E802 data does not exceed 30\%, then energy-momentum and baryon conservation alone require there to be an unexpected shoulder in the baryon spectrum in the region $2<y<3$. Our fits to the data in terms of a multicomponent firestreak model suggest surprisingly long stopping lengths, $L_{s} \approx 26 \mathrm{fm}$. Because these results deviate so much from previous expectations and analyses of more indirect data, systematic measurements of the $A$ and multiplicity dependence of $d N_{p} / d y$ over the full rapidity region should be undertaken to cross check these data and establish if indeed nuclei are as transparent as the present data seem to indicate. 


\title{
1.2 Nuclear Stopping Power at $15 \mathrm{GeV} /$ nucleon
}

by Scott Chapman and Miklos Gyulassy

(Published in Physical Review C 45 (1992) p. 2952)

\begin{abstract}
Fireball, firestreak and hadronic string models are shown to overpredict recent central $15 \mathrm{AGeV} \mathrm{Si}+\mathrm{Au}$ E802 spectrometer data. Claims in the literature about full nuclear stopping in $\mathrm{Si}+\mathrm{Au}$ reactions are therefore not supported by these data. In fact, fits to the spectrometer data indicate that up to half of the projectile nucleons may lose less than one unit of rapidity after traversing 5-10 fm of nuclear matter, implying an unexpected long stopping length of $\sim 20 \mathrm{fm}$. On the other hand, E810, E814, and preliminary E802 $d N_{\text {charged }} / d \eta$ data are more consistent with the expected degree of stopping.
\end{abstract}

\subsubsection{Introduction}

It has been claimed that "full stopping is realized[PANIC], showing a behavior close to the Landau model[Stach] and to relativistic fluid dynamics[Stau89], and the energy density can reach values comparable to the critical values for QGP formation"[Ame91]. However, as we pointed out in ref. [Chap91], the published E802 spectrometer data [E802] cast doubt on this belief, since in fact none of the present models is consistent with the full array of data. Moreover, if the spectrometer $d N / d y$ are normalized correctly, then these data are more indicative of a surprising degree of nuclear transparency. On the other hand, $d N_{\text {charged }} / d \eta$ [HIPAGS] and high rapidity E810[E810] and E814[E814] are well reproduced by models incorporating a high degree of nuclear stopping. As a result of this apparent inconsistency, no firm conclusion can yet be drawn on the important topic of the amount of nuclear stopping at the AGS.

In our letter[Chap91] we discussed a model independent fit to the spectrometer data which showed that if systematic errors do not cause more than a $30 \%$ suppression of proton and pion yields, then 4-momentum and baryon conservation laws imply that at least 11 out of 28 projectile nucleons suffer less than one unit of rapidity loss 
during a central $\mathrm{Si}+\mathrm{Au}$ collision. In this paper, we give the precise functional form of the fit used in the letter, as well as introducing three other fits which allow for the possibility of systematic errors in excess of $30 \%$. In addition to E802 spectrometer data[E802], we compare these four fits to E802 $d N_{\text {charged }} / d \eta$ data[HIPAGS] as well as data from the E810[E810] and E814[E814] collaborations. In our letter, we developed a multicomponent model $(\mathrm{mcm})$ in order to quantify the amount of nuclear stopping implied by the E802 spectrometer data. In this paper, in addition to explaining the mcm in more detail in the Appendix, we show that a simpler double firestreak model leads to similar conclusions about the amount of stopping. These types of models are only able to reproduce the spectrometer data with stopping lengths of $\sim 20 \mathrm{fm}$. In addition to central $\mathrm{Si}+\mathrm{Au}$ data, we discuss the agreement of these models with unpublished preliminary central $\mathrm{Si}+\mathrm{Al}$ and $\mathrm{Si}+\mathrm{Cu}$ E802 spectrometer data[Bloo], and make predictions for central $\mathrm{Au}+\mathrm{Au}$ proton and pion distributions at these same energies. The long stopping lengths implied by the E802 spectrometer data provide a sharp contrast to the results of $p+p$ and $p+A$ experiments at these same energies which imply stopping lengths of more on the order of 8-10 $\mathrm{fm}[\mathrm{pA}]$. Thus, either something new and unexplained is occurring in central $\mathrm{Si}+\mathrm{Au}$ collisions at the AGS, or else systematic errors in the spectrometer $d N / d y$ data must be significantly larger than previously estimated. In any case, the published E802 spectrometer data do not support claims of full nuclear stopping which are prevalent in the literature[PANIC, Stach, Ame91, RQMD91, RQMDpi, Brown, BrownEr].

\subsubsection{The Hadronic Fireball}

In the generic hadronic fireball model[Gosset], the projectile nucleus is assumed to be completely stopped by the target nucleus in the participant center-of-mass frame, whereupon thermal and chemical equilibrium are established. By treating both nuclei as hard spheres of constant baryon density $\left(\rho_{0}=.145 \mathrm{fm}^{-3}\right)$, geometry determines the number of interacting nucleons for any given impact parameter. For example, in 
a b=0 $\mathrm{Si}+\mathrm{Au}$ collision, all $28\left(=N_{p}\right)$ silicon nucleons interact with a central tube of about $75\left(=N_{t}\right)$ gold nucleons, thus making the baryon number of the resulting fireball $103\left(=N_{f}\right)$. The remaining 122 gold nucleons of this example are merely sF tators which are ignored in this model. Once $N_{p}$ and $N_{t}$ are known, the rapidity of the fireball rest frame and the total fireball energy in that frame are fixed by kinematics. For the $\mathrm{Si}+\mathrm{Au}$ example with $y_{p 0}=3.4$ and $y_{t 0}=0, y_{f}=1.3$ and $E_{f}=$ $250 \mathrm{GeV}$.

After its creation, the fireball expands and cools until freezeout, when the mean free path of the fireball hadrons becomes approximately the same size as the radius of the fireball. The temperature and chemical potentials at freezeout define the particle distributions according to

$$
f_{i}=\frac{d N_{i}}{d y d^{2} p_{\perp}}=\frac{\gamma_{i} g_{i} V_{f r} E}{\exp \left\{\left(E-B_{i} \mu-S_{i} \mu_{s}\right) / T\right\}-(-1)^{B_{i}}}
$$

where $B_{i}, S_{i}$ and $g_{i}$ are the baryon number, strangeness, and spin-isospin multiplicity for each species of hadron, $V_{f r}$ is the freezeout volume, and $\gamma_{i}$ is a parameter introduced to allow for incomplete the chemical equilibration. We assume that $\gamma_{i}=\gamma_{s}$ for all strange hadrons and $\gamma_{i}=1$ for all other hadrons. Since $E_{f}$ and $N_{f}$ are fixed by kinematics, $\mathrm{T}, \mu$ and $\mu_{s}$ can be found by choosing values for $\gamma_{s}$ and $V_{f r}$ (or $\left.\rho_{f r}=N_{f} / V_{f r}\right)$ and then solving the following; integral equations:

$$
\begin{gathered}
E_{f}=\sum_{i=\text { hadrons }} \int d^{2} p_{\perp} d y E f_{i} \\
N_{f}=\sum_{i=\text { baryons }} B_{i} \int d^{2} p_{\perp} d y f_{i} \\
0=\sum_{i=\text { strange }} S_{i} \int d^{2} p_{\perp} d y f_{i}
\end{gathered}
$$

We treat explicitly only the following hadronic resonances: $N, \Delta, \Lambda, \Sigma, \pi, \eta, \rho, \omega, \eta^{\prime}$, $K, K^{*}$ and their antiparticles. For example, for $b=0, \gamma_{s}=.5$ and $\rho_{f r}=5 \rho_{0}$, we find that $\mathrm{T}=200 \mathrm{MeV}, \mu=418 \mathrm{MeV}$ and $\mu_{s}=92 \mathrm{MeV}$ for AGS energy. 
Once T, $\mu$ and $\mu_{s}$ have been found for a given set of input parameters, $f_{i}\left(y, p_{\perp}\right)$ determine the invariant distributions for each species of hadron in the fireball. However, before reaching the detector, the heavy baryon and meson resonances decay as follows: $\Delta \rightarrow N+\pi, \Lambda \rightarrow p+\pi^{-}(64 \%$ of the time $), \Sigma^{+} \rightarrow p+\pi^{0}(52 \%), \Sigma^{+} \rightarrow n+\pi^{+}$ $(48 \%), \Sigma^{0} \rightarrow p+\pi^{-}(64 \%), \Sigma^{-} \rightarrow n+\pi^{-}, \eta \rightarrow 3 \pi(30 \%), \rho \rightarrow 2 \pi, \omega \rightarrow 3 \pi(90 \%)$, $\eta^{\prime} \rightarrow 2 \pi$, and $K^{*} \rightarrow K+\pi$, where the balance of the $\Lambda, \Sigma^{0}, \eta$ and $\omega$ decays are into undetected neutrals. For the $3 \pi$ decays, it is assumed for simplicity that each daughter particle carries away $1 / 3$ of the parent energy. By convoluting the above decays with parent distribution functions as in ref. [Das], the resonance contributions to the nucleon and pion distributions are found.

The net charge/baryon of the fireball is given by

$$
(Z / A)_{f}=\frac{\left(Z_{p} / A_{p}\right) N_{p}+\left(Z_{t} / A_{t}\right) N_{t}}{N_{f}}
$$

where $Z_{p}\left(Z_{t}\right)$ and $A_{p}\left(A_{t}\right)$ are the charge and atomic number of the projectile (targe $\hat{\imath})$ nucleus. Charge conservation is enforced as follows: All final state mesons not coming from strange baryon decays are assumed to be distributed isosymmetrically, and therefore the net charge carried by these mesons is determined solely by the kaon abundances:

$$
C_{m e s}=N_{K^{+}}-N_{K^{-}}
$$

From isosymmetry $\left(N_{K^{0}}=N_{K^{+}}\right.$, etc. $)$and conservation of strangeness before strange baryon decays, we have the relation $C_{m e s}=.5 Y$, where $\mathrm{Y}$ is the number of strange baryons in the fireball. It is assumed that all of the strange baryons have the same mass $(1.17 \mathrm{GeV})$ so that their relative abundances before decay do not depend on the temperature or chemical potentials. These abundances are taken to be $1 / 4$, $1 / 2(Z / A)_{f}, 1 / 4$, and $1 / 2\left(1-(Z / A)_{f}\right)$ for $\Lambda, \Sigma^{+}, \Sigma^{0}$, and $\Sigma^{-}$respectively. In this way, the net charge/baryon of all strange hadrons is always identical to the incoming charge/baryon ratio of $(Z / A)_{f}$. If, on the other hand, we had chosen $\Lambda$ 's and $\Sigma$ 's to have different masses, we would either need to introduce another chemical potential or 
some more complicated prescription for choosing strange baryon abundances in order to enforce charge conservation for arbitrary $\mathrm{T}, \mu$ and $\mu_{s}$. Finally, by demanding that $(Z / A)_{f}$ of the final nucleons not coming from strange baryon decays be protons, overall charge conservation can be enforced.

In the E802 experiment[E802], central $\mathrm{Si}+\mathrm{Au}$ events were identified by a high multiplicity trigger whose cross section $\left(=\sigma_{\text {cent }}\right)$ represented $7 \%$ of the total $\mathrm{Si}+\mathrm{Au}$ inelastic cross section $(=3822 \mathrm{mb}[\mathrm{Bloo}])$. In our model, we chose a maximum impact parameter $\left(b_{\max }=2.9 \mathrm{fm}\right)$ such that $\pi b_{\max }^{2}=\sigma_{c e n t}$ and then integrated our fireball results over b from 0 to $b_{\max }$.

In the experiment, measurements were made using a spectrometer arm with a range of $5^{\circ}<\theta<55^{\circ}$ which could detect and identify charged particles with total momentum between 0.5 and $3 \mathrm{GeV} / \mathrm{c}[\mathrm{E} 802, \mathrm{E} 802 \mathrm{a}]$. The resulting raw particle distributions were binned both in $\mathrm{y}$ and $m_{\perp}$. For each rapidity bin, the distributions appear to be well fit by pure exponentials in $m_{\perp}$ [E802]:

$$
d N_{i} / d y d^{2} m_{\perp}=\rho_{i}(y) \exp \left(-\left(m_{i \perp}-m_{i}\right) / T_{i}(y)\right)
$$

The rapidity distributions were then estimated by integrating these fits over $m_{\perp}$ :

$$
d N_{i} / d y=2 \pi \rho_{i}(y) T_{i}(y)\left(T_{i}(y)+m_{i}\right)
$$

In the fireball model, $d N_{i} / d y$ can be calculated in two ways: by numerically integrating $f_{i}$ over all $d^{2} m_{\perp}$ or by using the exponential fitting procedure outlined above after imposing the experimental phase space constraints. For all of our calculations, the difference between the results of these two methods was less than $20 \%$ for $d N_{\pi} / d y$ and completely negligible for $d N_{p} / d y$.

In fig. 1.2.1 we compare three fireball models to the data. The solid dots are data from the E802 spectrometer[E802], while the diamonds and x's are data from E810[E810] and E814[E814] respectively. It should be noted that the E814 data are actually for $S i+P b$ collisions rather than $S i+A u$ and that the three experiments 
Fireballs
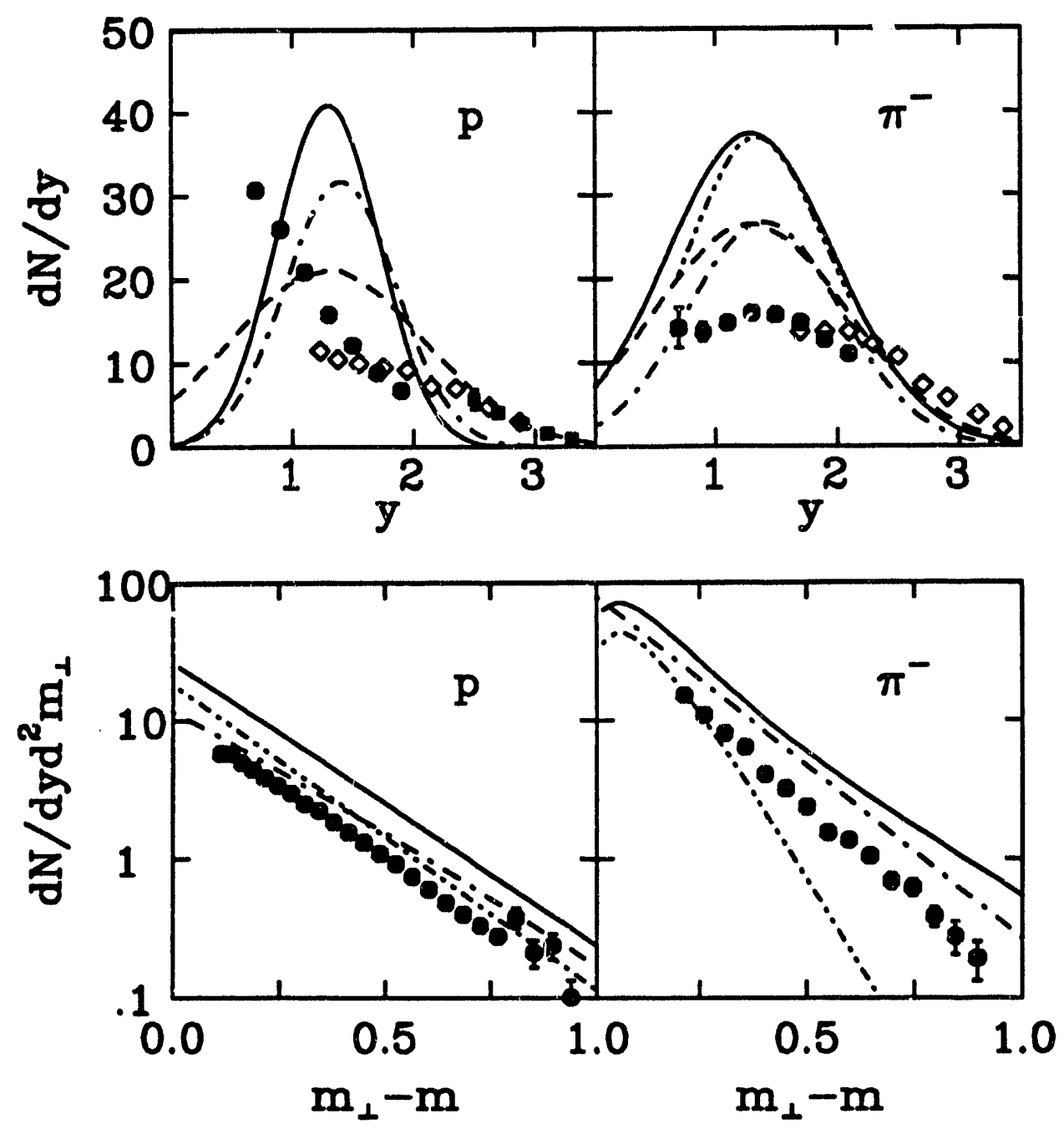

Figure 1.2.1: The solid dots in the upper panels show the proton and $\pi^{-}$rapidity distributions measured by the E802 spectrometer in $14.6 \mathrm{AGeV} / \mathrm{c}$ central $S i+A u$ reactions, while the bottom panels show the $m_{\perp}$ distributions for $y=1.3$ in those same reactions[E802]. The diamonds show E810 ' + ' - '-' and negatively charged particle distributions for $\mathrm{Si}+\mathrm{Au}$ using a trigger with twice the cross section of the E802 central trigger[E810]. The solid squares are E814 proton data for central $\mathrm{Si}+\mathrm{Pb}$ reactions[E814]. Solid curves show results of the generic fireball model, while dashed and dot-dashed curves denote Landau hydrodynamic fireball[Stach] and hydrochemical fireball[Brown, BrownEr] results respectively. The norms of the hydrochemical results have been adjusted in accordance with the published erratum[BrownEr]. The dot-dot-dashed curves in the lower panels show $m_{\perp}$ distributions of protons and $\pi^{-}$'s coming only from heavy baryon decays in the generic fireball model. The dot-dotdashed curve in the upper right panel shows the generic fireball prediction for the pion rapidity distribution given the restricted phase space of the experiment. 
use different centrality triggers (the E810 trigger has twice the cross section of the E802 trigger, while the E814 trigger has less than half the cross section of E802). Nevertheless, comparing these data sets to one another is done quite often[Nag] and is useful for making qualitative cross checks. The solid line in fig. 1.2.1 shows the results of the generic fireball model outlined above with $\rho_{f r}=5 \rho_{0}$ and $\gamma_{s}=.5$. This fireball model produces more than a factor of 2 too many protons, pions, and kaons (not shown) at mid-rapidity. Using a higher freezeout baryon density results in more heavy baryon resonances and slightly fewer pions, but the increased temperature makes the distributions become too broad in $m_{\perp}$. Increasing (decreasing) $\gamma_{s}$ increases (decreases) the number of kaons and strange baryons but does not have a significant effect on the total number of mid-rapidity protons and pions. In fact, no reasonable variation of $\rho_{f r}$ and/or $\gamma_{s}$ significantly improves agreement with the data. In addition to the generic fireball, fig. 1.2.1 also shows results from the Landau hydrodynamic longitudinally expanding fireball[Stach] (dashed line) and the hydrochemical spherically expanding fireball[Brown, BrownEr] (dot-dashed line). The longitudinal expansion of the Landau fireball reduces the midrapidity proton and pion peaks but still overpredicts the E802 proton data by at least $70 \%$ in the range $1.5<y<2$. On the other hand, it should be noted that this model does a very good job of reproducing high rapidity E810 and E814 data. Even though the spherical expansion of the hydrochemical model provides a possible explanation for the difference in proton and pion slopes, this model also fails to reproduce the measured norms of these distributions. In fact, all of the fireball models considered here overpredict the E802 proton and/or pion rapidity distributions by at least $70 \%$ in some rapidity range.

It has been suggested[Brown, BrownEr] that at least some of the discrepancy in $d N_{\pi} / d y$ could be due to an unmeasured excess of low $p_{\perp}$ pions coming from baryon resonance decays. The dot-dot-dashed curves in the bottom two panels of figure 1.2.1 show the distributions of protons and pions coming only from baryon resonance decays in the generic fireball model. At least for the generic fireball, any low $p_{\perp}$ enhancement 
due to these resonances is entirely negligible for protons and less than $20 \%$ for pions, as can be seen by comparing the restricted, exponentially fitted $d N_{\pi} / d y$ (dot-dot-dashed line in fig. 1.2.1) with the directly calculated $d N_{\pi} / d y$ (solid line). Furthermore, even if one makes the assumption of the hydrochemical model[Brown, BrownEr] that none of the pions coming from baryon resonance decays are detected, fireball models still predict $70 \%$ more mid-rapidity pions than are seen in the data (dashed line in fig. 1.2.1). Since none of the fireball models discussed here can simultaneously reproduce all of the data, we turn to other models.

\subsubsection{The Firestreak and String Models}

The firestreak[Gosset, Myers] model was designed to take into account the diffuse edges of colliding nuclei by creating many smaller scale regions of local equilibrium rather than a single large fireball. In this model, the projectile and target nuclei are divided into longitudinal tubes with transverse area $a_{\perp}\left(\lesssim 1 \mathrm{fm}^{2}\right)$. Each set of two opposing tubes forms a completely stopped miniature fireball (or firestreak) in its local center of mass frame. In this way a large number of independent firestreaks forms, each with its own local values of $N_{f}, y_{f}, \mathrm{~T}, \mu$ and $\mu_{s}$. As a result of this locality, Wood-Saxon density distributions rather than sharp spheres can be used to determine how many nucleons are in each tube. Often, some very asymmetric cases will result. For example, a tube containing 3 nucleons from the center of a gold nucleus could interact with a tube containing .1 nucleon from the diffuse edge of a projectile silicon nucleus to create a streak with $N_{f}=3.1$ and $y_{f}=0.4$. These asymmetries provide a natural way to generate low-rapidity "spectator" contributions, even though there are no true spectators in this model.

Hadronic string models[Lund] also feature locality, though they do not impose the requirement of complete nuclear stopping. In fig. 1.2.2, we compare the firestreak (dashes) and two string models (Attila[Gyu87] (solid) and QGSM[Ame91] (histogram)) with the data. For the Firestreak and Attila, we have calculated $T_{i}(y)$ 


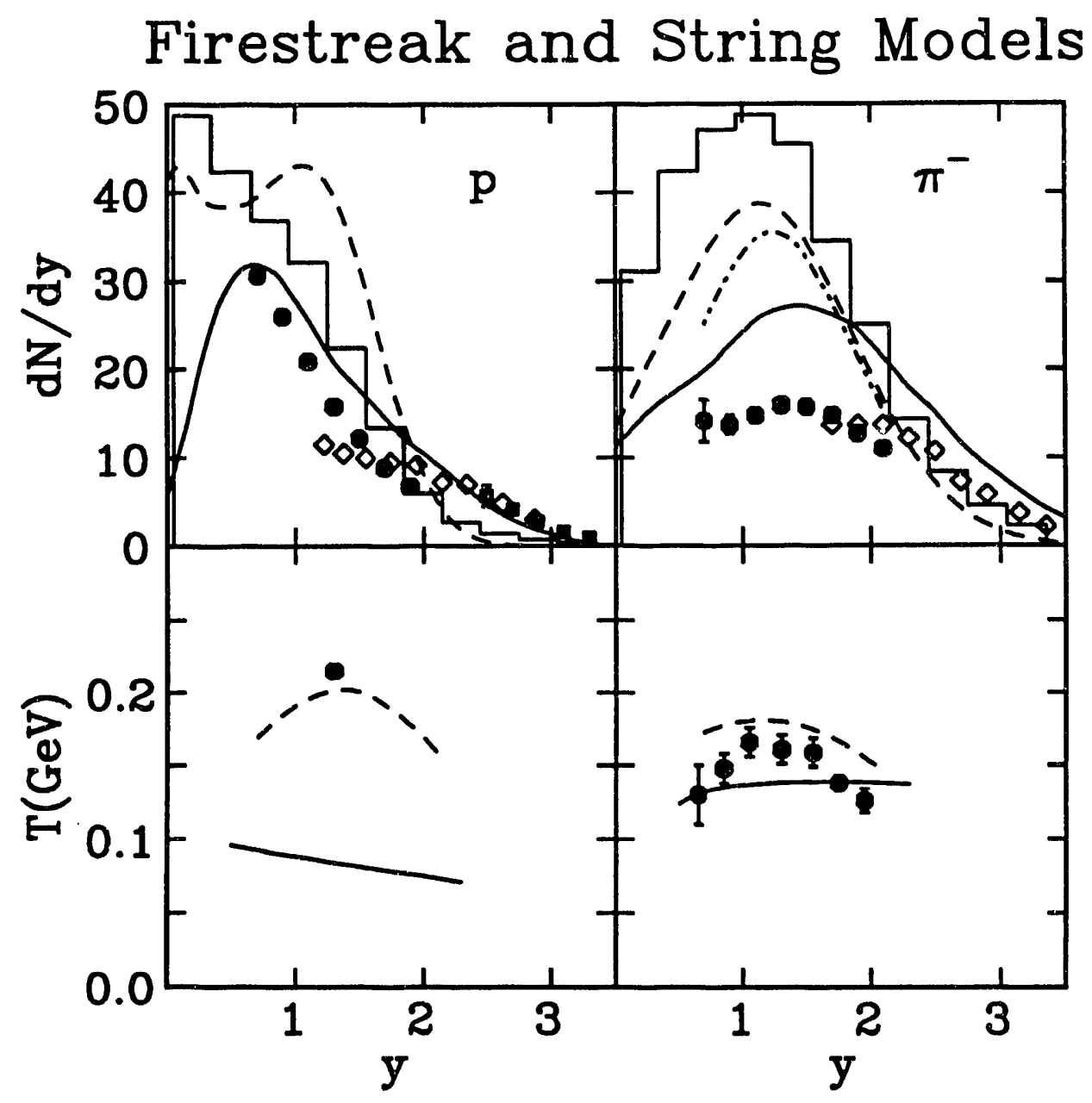

Figure 1.2.2: The top panels are as in fig. 1.2.1, while the bottom panels are the inverse slope parameters of eqn. (1.2.7)[E802]. These data are compared to firestreak[Myers] (dashed), Attila[Gyu87] (solid), and QGSM[Ame91] (histogram) calculations. The dot-dot-dashed curve in the upper right panel shows the firestreak prediction with experimental phase space restrictions.

via the exponential fitting procedure of eqn. (1.2.7) in order to compare our curves to the published $T_{i}(y)$ values. Though the firestreak improves on the fireball by showing "spectator" contributions, it still has the problem of predicting far too many midrapidity protons and pions, even after the experimental acceptance has been folded in (dot-dot-dashed line in $d N_{\pi} / d y$ ). The string models do a better job of reproducing the overall ramp shape of $d N_{p} / d y$, though they still overpredict by at least $70 \%$ the number of pions seen by E802. It should be noted that although Attila overpredicts 
by about $50 \%$ the high rapidity protons seen by E802, it reproduces those seen by E810 and E814 very well.

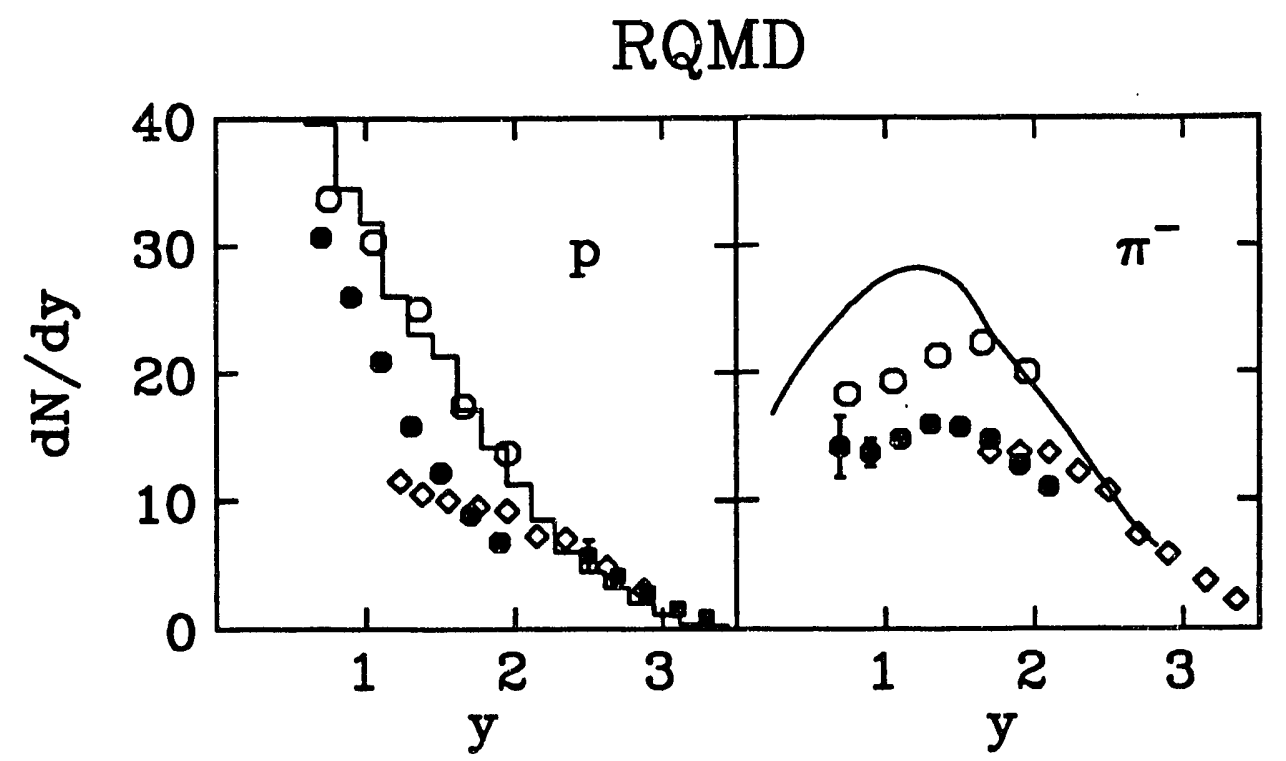

Figure 1.2.3: The data of the top of fig. 1.2.1 are compared to RQMD calculations by $\mathrm{H}$. Sorge et al. (histogram[RQMD92] and solid[RQMDpi]) and to RQMD calculations which have explicitly incorporated the experimental acceptances and cuts (open circles)[RQMD802].

Recently, there have been claims[RQMD802] that the RQMD model[RQMD91] is consistent to within $23 \%$ with the E802 spectrometer data. In fig. 1.2.3, we compare various RQMD runs with proton and pion rapidity data. Since H. Sorge et. al. have not yet published proton and $\pi^{-}$rapidity distributions in the same paper, we show the proton distribution from ref.[RQMD92] (histogram) and the $\pi^{-}$distribution from ref. [RQMDpi] (solid curve). These curves consistently overpredict the E802 data, even by as much as $70 \%$ for midrapidity pions. The open circles in fig. 1.2 .3 represent the results of an RQMD run which was subjected to the E802 experimental acceptances and cuts[RQMD802]. It is interesting that this RQMD run still overpredicts the E802 pion and proton yields by $\sim 50 \%$ and $\sim 70 \%$ respectively in the region $1.5<y<2$. None of these discrepancies can be due to undetected low $p_{\perp}$ components since the same exponential fitting procedure was used for this RQMD run as for the E802 data. 
On the other hand, RQMD does a very good job of reproducing the high rapidity E810 and E814 data.

\subsubsection{Model Independent Fits}

Having seen that none of the above equilibrium and nonequilibrium models for nuclear collision dynamics are able to simultaneously reproduce all of the published data, we consider next a model independent fitting procedure in order to isolate possible causes for the discrepancies. We begin by fitting the experimental $T_{i}(y)$ [Bloo] and $(d N / d y)_{i}(y)[E 802]$ data with simple functions which have reasonable extrapolations to phase space regions outside of the experimental acceptance. Equations (7) and (8) are then used to determine the invariant distributions, $f_{i}=d N_{i} / d y d^{2} m_{\perp}$, from which information about momentum and energy conservation can be extracted.

For the meson $(d N / d y)_{i}(y)$ we use

$$
(d N / d y)_{i}=\alpha C_{i} \exp \left(-\left(y-y_{i}\right)^{2} / \delta_{i}\right) \quad-1 \leq y \leq 4
$$

where $\left(C_{i}, y_{i}, \delta_{i}\right)$ are fit with $(16,1.4,1),(16,1.35,1.3),(3.5,0.95,1)$ and $(0.67,1.3$, 1) for $\pi^{+}, \pi^{-}, K^{+}$and $K^{-}$respectively. The reported data are fit with $\alpha=1$, but later we set $\alpha=1.3$ to account for experimental systematic errors. The meson and proton temperatures are given by:

$$
\begin{aligned}
T_{\pi^{+}} & =T_{\pi^{-}}=0.06+.1 \exp \left(-(y-1.3)^{2} / 1.2\right)+0.03 \exp \left(-y^{2}\right) \\
T_{K^{+}} & =T_{K^{-}}=0.19 \exp \left(-(y-1.3)^{2} / 2 .\right) \\
T_{p} & = \begin{cases}0.23 \exp \left(-(y-1.55)^{2}\right)+.1 \exp \left(-y^{2}\right) & y<2.2 \\
0.15 & y>2.2\end{cases}
\end{aligned}
$$

We fit the proton rapidity spectrum with a falling quadratic ramp and include adjustable undetected spectator and projectile gaussians in order to conserve baryon 
number and to test for transparency:

$$
(d N / d y)_{p}=\alpha \begin{cases}C_{s p e c} e^{\left(-y^{2} / \delta_{\text {spec }}\right)} & -1<\hat{\mathrm{y}}<0 \\
\operatorname{Max}\left[\begin{array}{l}
C_{\text {spec }} e^{\left(-y^{2} / \delta_{\text {spec }}\right)} \\
6 y^{2}-35 y+52+C_{\text {pro }} e^{\left(-\left(y-y_{\text {pro }}\right)^{2} / \delta_{\text {pro }}\right)}
\end{array}\right] & 0<y<3 \\
7-2 y+C_{\text {pro }} e^{\left(-\left(y-y_{\text {pro }}\right)^{2} / \delta_{\text {pro }}\right)} & 3<y<3.5 \\
0 & \text { otherwise }\end{cases}
$$

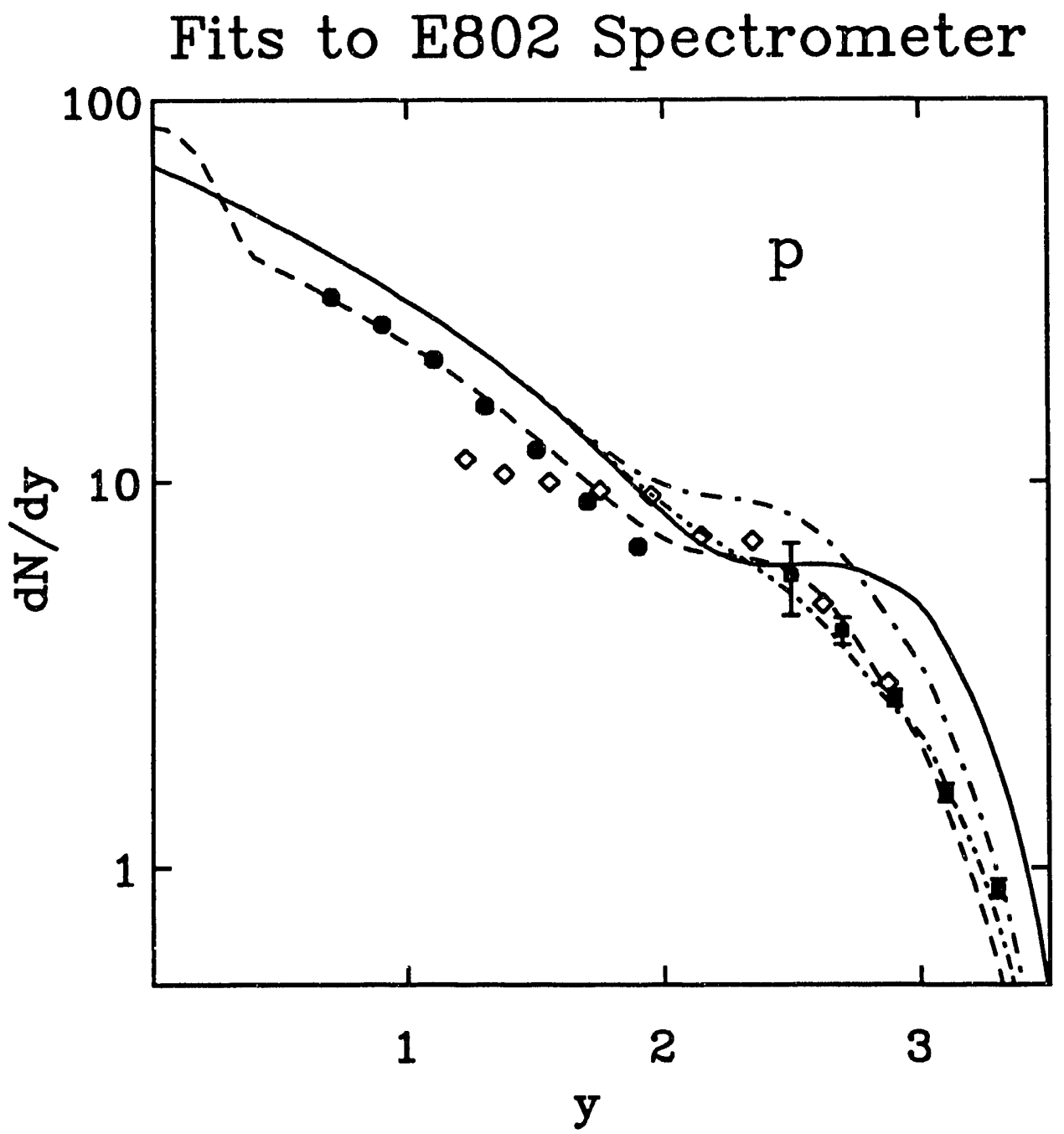

Figure 1.2.4: 'The same proton data as in fig. 1.2.1 plotted on a log scale. In addition to a datafit (dashed) which does not conserve 4-momentum, we show fit1 (solid), fit2 (dot-dashed), fit3 (dot-dashed), and fit4 (dot-dot-dashed). 


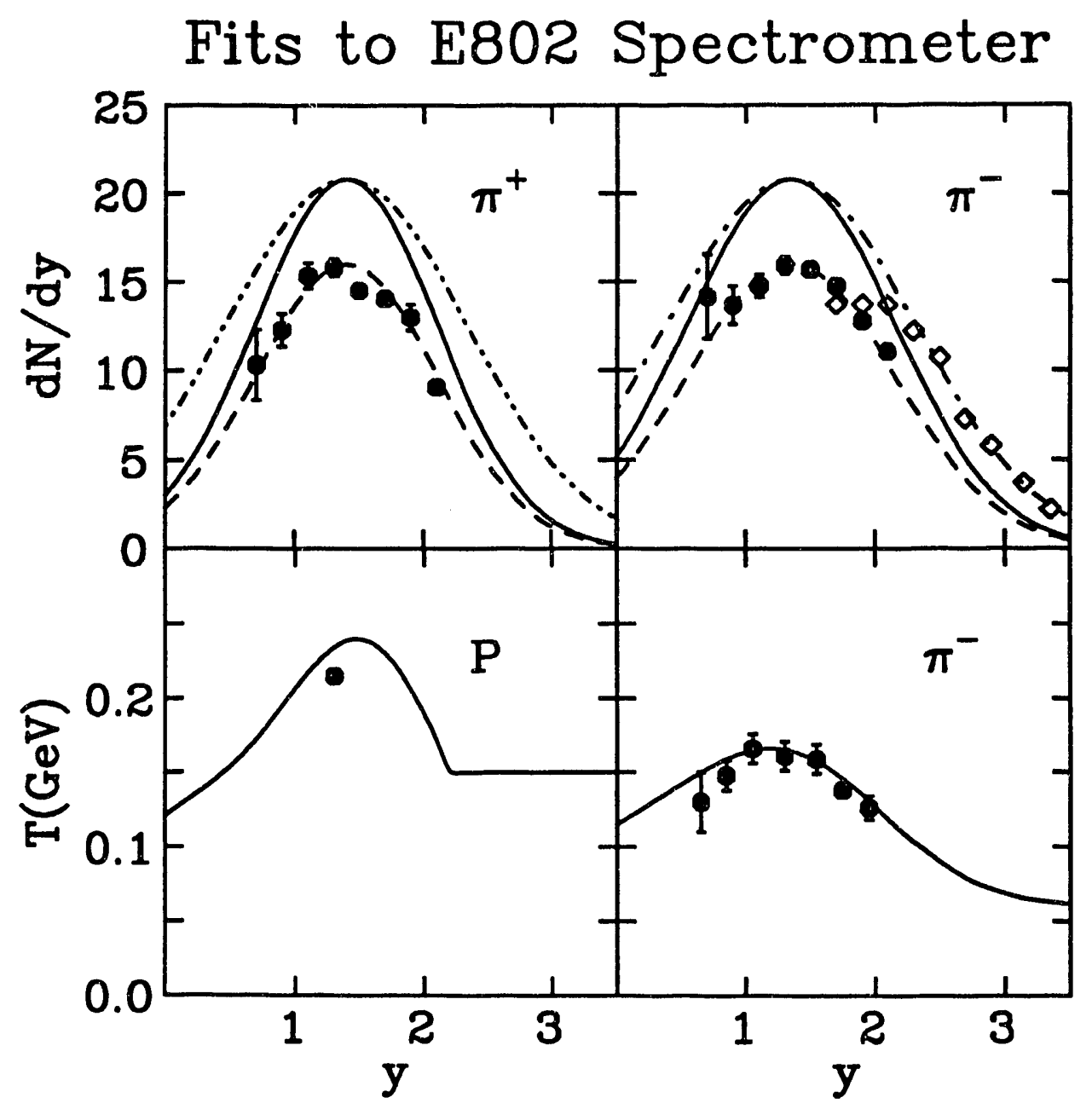

Figure 1.2.5: Data as in fig. 1.2.2 with E802 $d N_{\pi^{+}} / d y[\mathrm{E} 802]$ instead of protons. Also in the upper panels we show fit1 (solid), fit2 (solid), fit3 (solid $\pi^{+}$and dot-dashed $\pi^{-}$) and fit4 (dot-dot-dashed $\pi^{+}$and dot-dashed $\pi^{-}$). The lower panels show the $\mathrm{T}(\mathrm{y})$ which were used for all of the fits.

where $\delta_{\text {pro }}=0.2$. For the unobserved neutral mesons it is assumed that $\pi^{0}=\left(\pi^{+}+\right.$ $\left.\pi^{-}\right) / 2, K^{0}=K^{+}$, and $\bar{K}^{0}=K^{-}$. Charge conservation is enforced by demanding that the total number of final protons be $N_{p}=14+79-N_{\pi^{+}}-N_{K^{+}}+N_{\pi^{-}}+N_{K^{-}}(=91.9$ for the above fit). We employ E810 and E814 data to guide our $d N_{p} / d y$ extrapolation to high rapidities by using $\left(y_{\text {pro }}, C_{\text {pro }}, C_{s p e c}, \delta_{s p e c}\right)=$ datafit $=(2.5,3.8,80.9,0.17)$, where the last two parameters were chosen to get the right value for $N_{p}$. With $N_{p}$ 


\section{Fits to E802 Spectrometer}
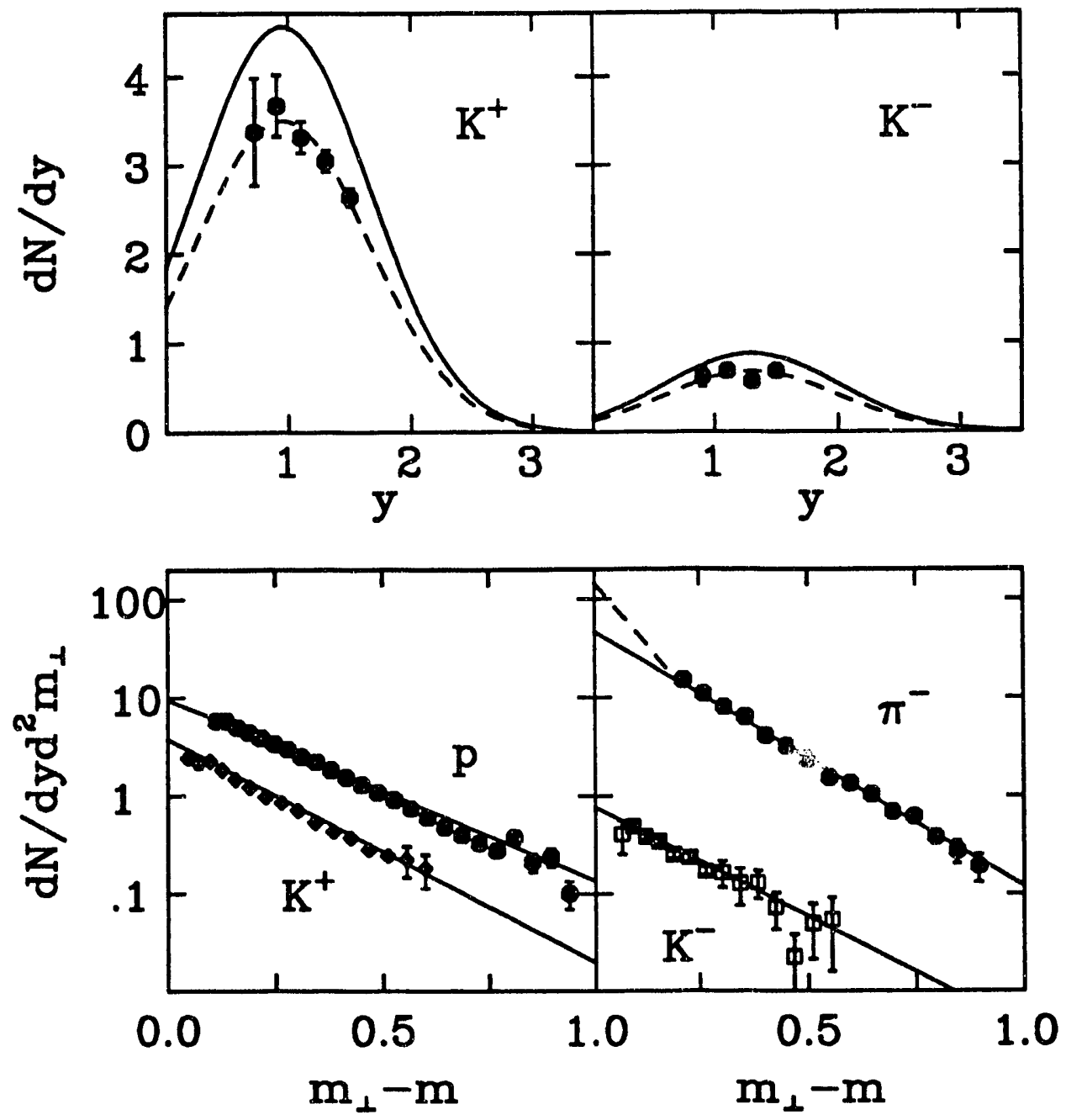

Figure 1.2.6: The upper panels show the E802 $K^{ \pm}$rapidity distributions in central $S i+A u$ reactions (solid dots), while the bottom panels show $m_{\perp}$ distributions for $y=1.3$ in these same reactions[E802]. Simple fits to the data are shown by dashed curves in the upper panels and solid lines in the lower panels. The solid curves in the upper panels show the $30 \%$ enhancement used in fit 1 - fit4. The dashed line in the lower right panel shows a low $m_{\perp}$ component which would give rise to a $30 \%$ systematic error in $d N_{\pi} / d y$.

fixed, the total number of undetected neutrons is given by baryon number conservation, $N_{n}=28+197-N_{p}=133.1$. The correct value for $N_{n}$ can be achieved by assuming an $\mathrm{n} / \mathrm{p}$ ratio of 1.3 for $y>2$ (based on E814 findings[Stach]) and $\mathrm{n} / \mathrm{p}=1.46$ for $y<2$. This fit to the three experiments at the AGS allows us to take into account 
all of the observed energy in longitudinal and transverse motion as well as pion and kaon production. Datafit is shown by the dashed lines in figs. 1.2.4, 1.2.5 and 1.2.6 (solid lines for the temperatures).

The total outgoing longitudinal momentum $P_{z}$ implied by this fit is easily calculated by integrating $m_{\perp} \sinh (y) f_{i}$ over $d^{2} m_{\perp}$ and $\mathrm{y}$ :

$$
P_{z}=\sum_{i=\text { hadrons }} \int d y \frac{2 T_{i}^{2}+2 T_{i} m_{i}+m_{i}^{2}}{T_{i}+m_{i}}(d N / d y)_{i} \sinh (y) .
$$

$\mathrm{E}$ is simply found by replacing $\sinh (\mathrm{y})$ by $\cosh (\mathrm{y})$. For datafit, the integration over y gives $P_{z}=289 \mathrm{GeV} / \mathrm{c}$ and $\mathrm{E}=495 \mathrm{GeV}$, whereas the total incoming energy and momentum are known to be $P_{z}=409 \mathrm{GeV} / \mathrm{c}(=28 \times 14.6)$ and $\mathrm{E}=595 \mathrm{GeV}$ $(=197 \times .939+28 \times 14.63) .120 \mathrm{GeV} / \mathrm{c}$ of the incoming momentum and $100 \mathrm{GeV}$ of the energy are unaccounted for in this fit to the data! If we assume that neither leptons nor photons carry a significant fraction of the 4-momentum, then there must be some undetected hadrons somewhere which do carry it. The E802 collaboration noted that an undetected excess of low $p_{\perp}$ particles could result in a $25 \%$ enhancement of $d N / d y$ over the exponentially fitted data[E802]. To take this into account as well as other possible systematic errors in the data, we multiply each of our $(d N / d y)_{i}$ functions by $\alpha=1.3$ and adjust $C_{\text {pro }}$ to 2.3 in order to preserve high rapidity agreement with E810 and E814 ( $C_{\text {spec }}=43.9$ for charge conservation). Even with this $30 \%$ enhancement over all of the E802 data, we find that $50 \mathrm{GeV} / \mathrm{c}$ of momentum and $45 \mathrm{GeV}$ of energy are still missing.

It seems that either the true $d N_{i} / d y$ exceed the published E802 data by more than $30 \%$ in some rapidity regions, or else the "missing" 4-momentum must be carried by more high rapidity hadrons than we use in the above extrapolations. If we assume that the latter is true, then the least transparent solution which does not overpredict any of the spectrometer data by more than $30 \%$ has fit $1=(2.75,3.4,40.9,0.17)$, where now a constant $\mathrm{n} / \mathrm{p}$ ratio of 1.46 is assumed throughout and $\left(\alpha, \delta_{\text {pro }}\right)=(1.3, .25)$ for the rest of the fits considered in this paper. This 4-momentum conserving fit (solid lines in figs. 1.2.4-1.2.6) overpredicts high rapidity E810 and E814 proton data 
by a factor of 2 . By allowing a $50 \%$ disagreement with the last proton data point, a slightly less transparent solution can be found: fit2 $=(2.5,4.29,32.28, .25)$ which only overpredicts E810 and E814 by 50\% (dot-dashed curve in fig. 1.2.4). These solutions have 10.8 and 10.1 nucleons respectively in the projectile region $(2.44<y<3.5)$. In the lower half of fig. 1.2.6 we show how an undetected low- $m_{\perp}$ component for pions could give rise to a $30 \%$ normalization error in $d N_{\pi} / d y$. However, since a high- $m_{\perp}$ hadron with rapidity $y$ carries more 4 -momentum than a low- $m_{\perp}$ hadron with the same rapidity, it is more conservative to use a uniform $30 \%$ enhancement everywhere as we did in our calculations.

If we allow a $40 \%$ disagreement with the last two E802 $\pi^{-}$data points, the width of the $d N_{\pi^{-}} / d y$ Gaussian can be increased to $\delta_{\pi^{-}}=1.85$ to provide agreement with E810 negatives at high rapidities (dot-dashed $d N_{\pi} / d y$ in fig. 1.2.5). By using fit3 $=(2.5,4.58,72.7,0.07)$ to define the proton distribution, 4-momentum can be conserved with 9.7 nucleons in the projectile region. The $y>0$ protons in this fit are distributed almost identically to the protons in fit2, though from charge conservation

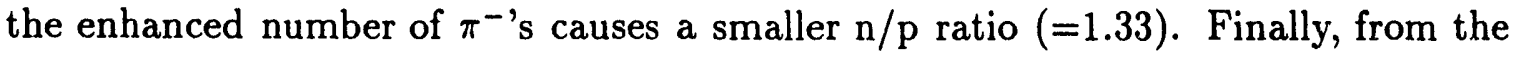
fact that silicon is isosymmetric, one could argue that high-rapidity pions should be isosymmetric and therefore that the $\pi^{+}$'s should also be distributed like the E810 negatives at high rapidities. This can be achieved by taking $\delta_{\pi^{+}}=1.75$ and fit $4=(2.5$, $1.92,49.3,0.09$ ), which has only 6.6 nucleons in the projectile region and is shown by the dot-dot-dashed $d N_{p} / d y$ and $d N_{\pi} / d y$ curyes in figs. 1.2.4 and 1.2.5. Though this fit conserves 4-momentum and agrees well with high rapidity E810 and E814 proton data, it disagrees with the last two E802 $\pi^{+}$data points by $70-100 \%$ and it features an $\mathrm{n} / \mathrm{p}$ ratio of 1.56 even in the projectile region.

It is instructive to compare the four fits discussed above to other preliminary data from E802 as well as to leading neutron data from E814. In addition to the spectrometer arm, E802 has a target multiplicity array (TMA) detector which measures $d N / d \eta$ of charged particles and a beam calorimeter (ZCAL) which measures the resid- 


\section{Charged Particles}

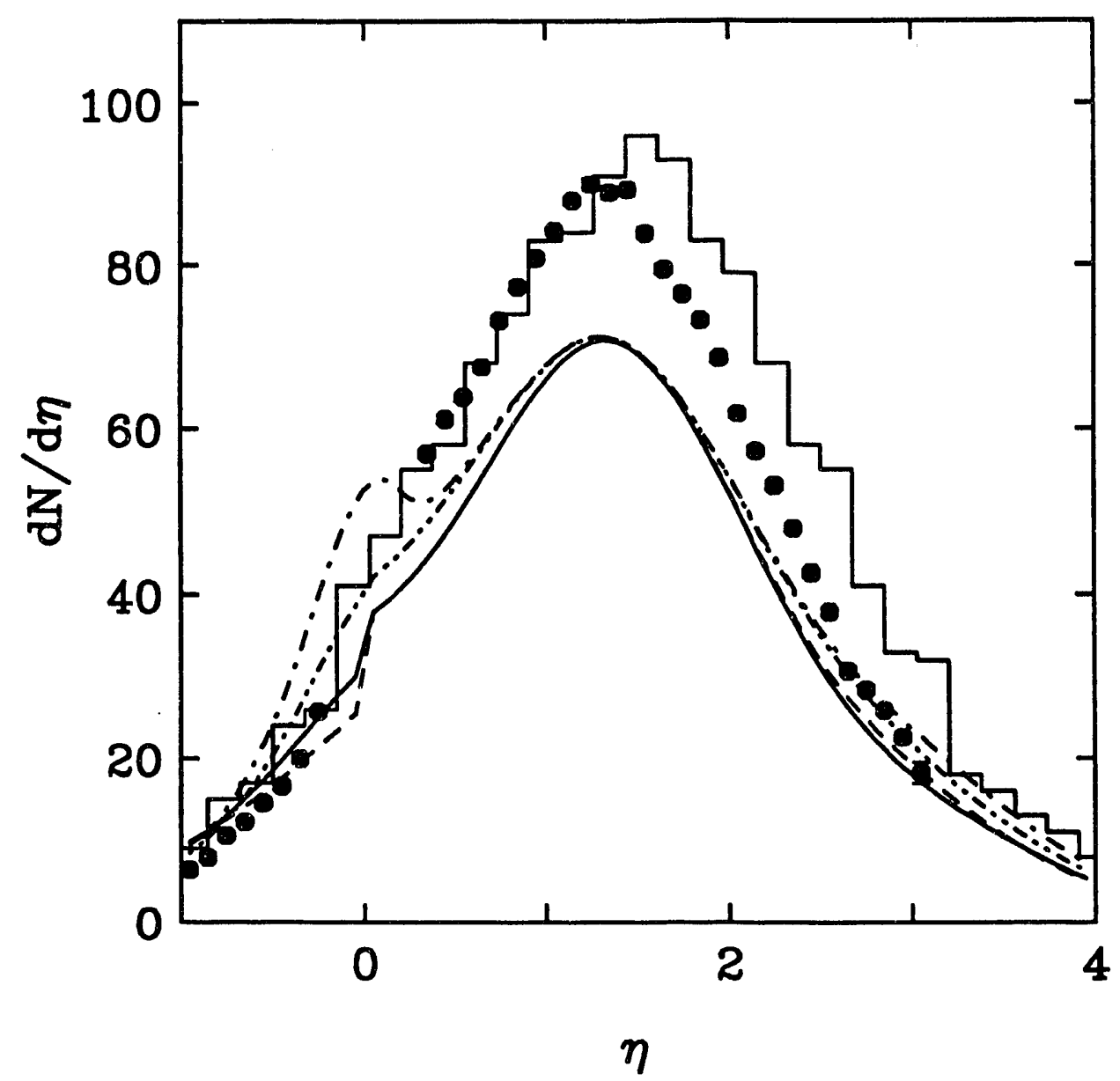

Figure 1.2.7: Preliminary $d N_{\text {charged }} / d \eta$ data[HIPAGS] are compared to results of RQMD [RQMD91] (histogram), fit1 (solid), fit2 (dashed), fit3 (dot-dashed), and fit4 (dot-dot-dashed).

ual beam energy after a collision. Due to the geometry of the ZCAL detector, there is some uncertainty as to whether it measures the energy of final particles with $\theta<0.8^{\circ}$ or with $\theta<2.2^{\circ}$ [Bloo]. For $\theta_{\max }=0.8^{\circ}$ the four fits discussed above give $\mathrm{ZCAL}$ energies (in $\mathrm{GeV}$ ) of $(5.3,3.8,4.4,4.1)$, while for $\theta_{\max }=2.2^{\circ}$ these same fits give (37.0, $27.4,30.6,27.6)$. If $\theta_{\max }$ is indeed $0.8^{\circ}$, then none of the above fits are inconsistent with correlations between the TMA (which defines the central trigger) and ZCAL measurements[Bloo]. In fig. 1.2.7 we compare $d N / d \eta$ distributions from the four fits 
and RQMD[RQMD91] with preliminary TMA data[HIPAGS]. It is interesting that the four fits, each of which exceed the spectrometer multiplicities by at least $30 \%$, still underestimate the TMA multiplicity. RQMD, on the other hand, can reproduce $d N / d \eta$ quite well even though it overpredicts spectrometer yields by $50-70 \%$ in some rapidity regions. Since no reasonable fit or model can simultaneously reproduce both the spectrometer and TMA charged particle multiplicities, there appears to be some inconsistency between these two data sets. We note here that preliminary E814 $d N / d \eta$ data are in very good agreement with the E802 TMA data[E814a].

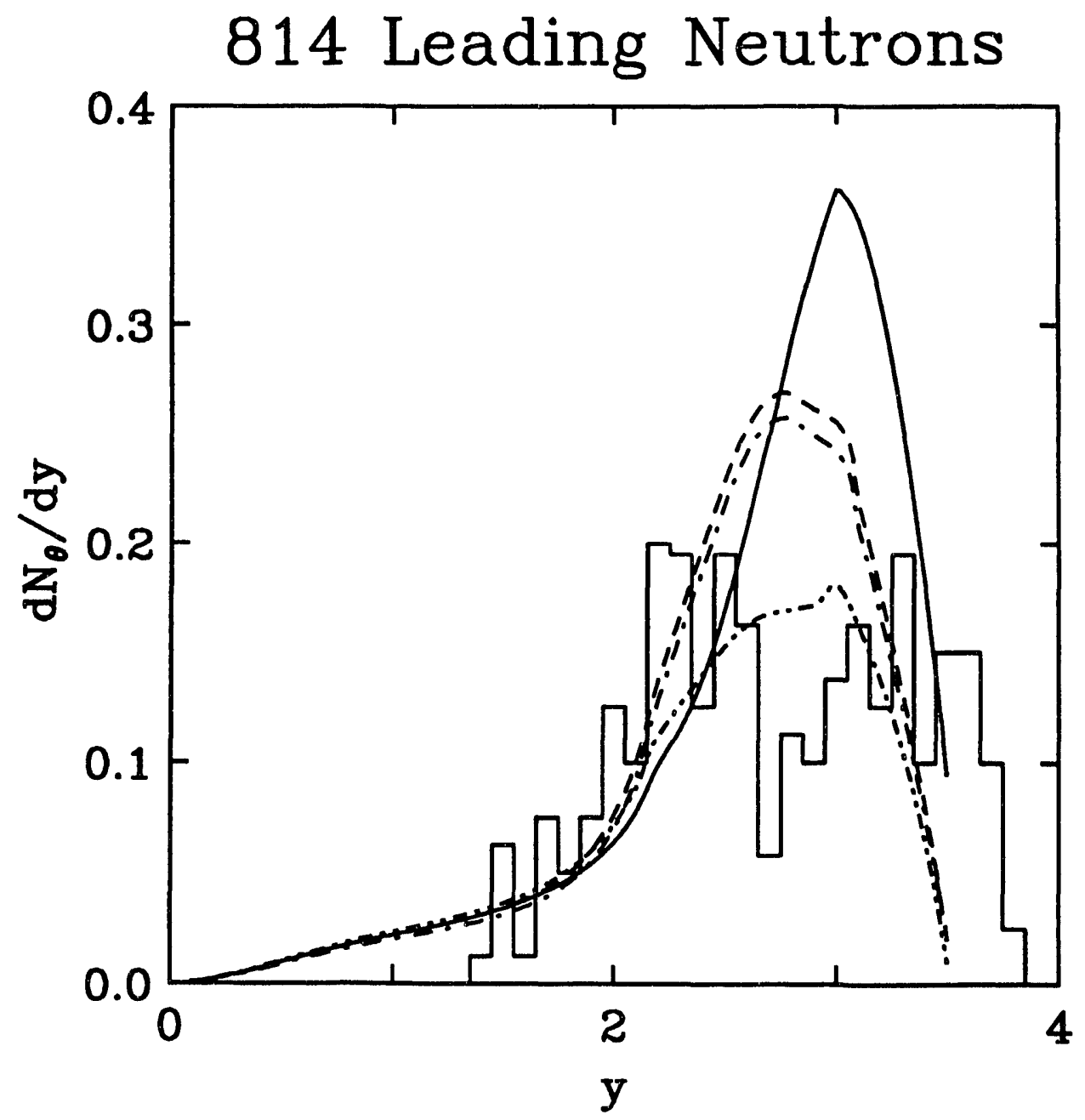

Figure 1.2.8: The histogram shows the rapidity distribution for neutrons emerging with a beam angle of less than $0.8^{\circ}$ in central $\left(E_{\perp 814}>13 \mathrm{GeV}\right) \mathrm{Si}+\mathrm{Pb}$ collisions[E814]. Fit1 (solid), fit2 (dashed), fit3 (dot-dashed), and fit4 (dot-dot-dashed) for central $\mathrm{Si}+\mathrm{Au}$ are compared to these data. 


\section{Particle Calorimeter}

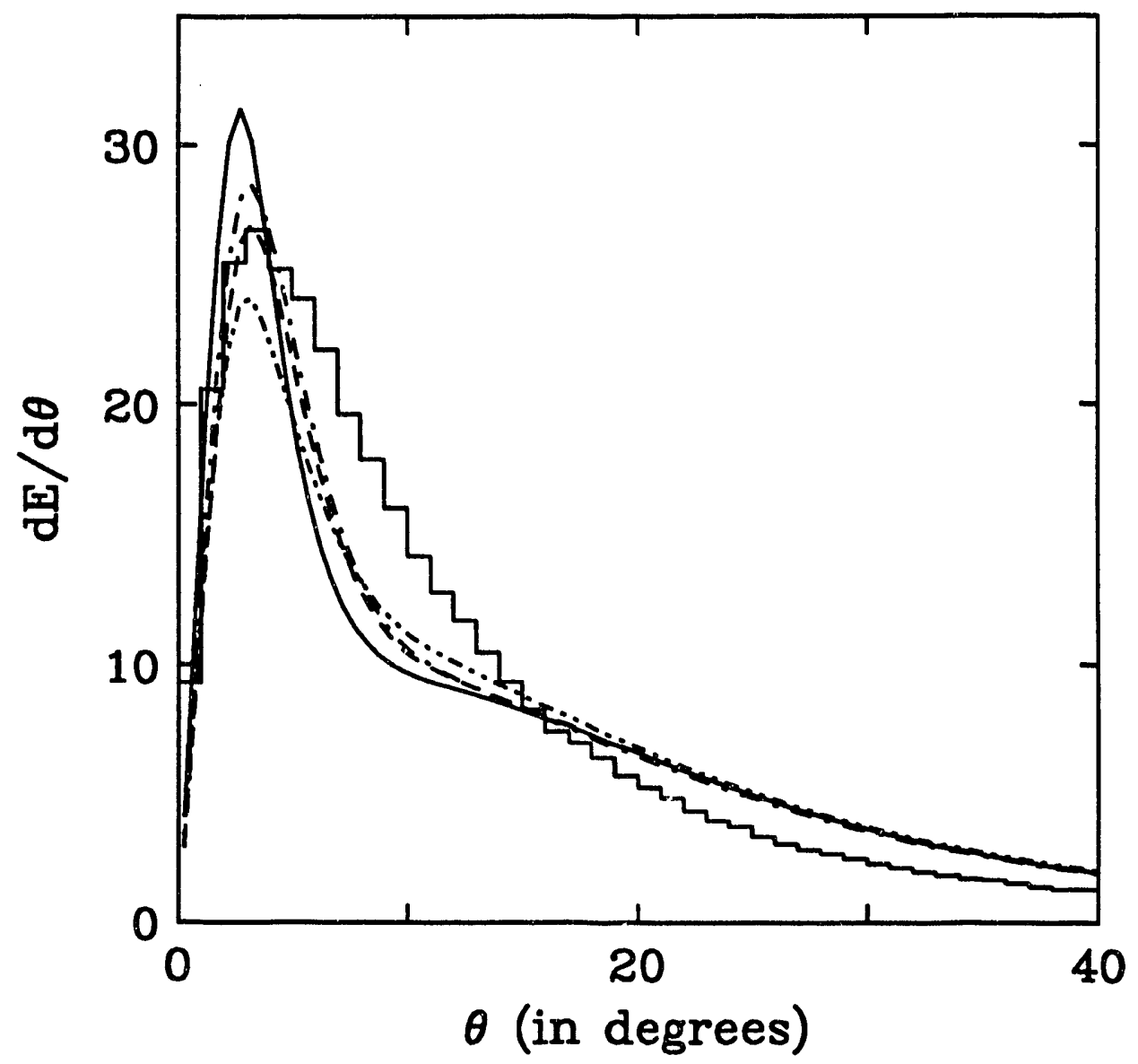

Figure 1.2.9: Angular energy distributions (kinetic energy for baryons) are shown for Attila[Gyu87] (histogram), fit1 (solid), fit2 (dashed), fit3 (dot-dashed), and fit4 (dot-dot-dashed).

In the $\mathrm{E} 814$ experiment, neutrons emerging from $\mathrm{Si}+\mathrm{Pb}$ collisions with a beam angle of $\theta<0.8^{\circ}$ are measured using a forward spectrometer[E814]. Their rapidity is determined by the amount of energy that they deposit in the spectrometer, and so a $d N_{\theta} / d y$ plot of neutrons having $\theta<0.8^{\circ}$ is generated. In fig. 1.2 .8 we compare $d N_{\theta} / d y$ from our four fits with leading neutron data for central $(\sigma \sim 40 \mathrm{mb}) \mathrm{Si}+\mathrm{Pb}$ collisions [E814]. The agreement is best for fit4, but due to the statistical uncertainty of the data as well as the different target $(\mathrm{Pb})$ and trigger used by E814, none of the fits can be 
ruled out. In addition to leading neutrons, E814 also measures $d E / d \theta[$ Fox]. Though these data are not yet published, we have plotted $d E / d \theta$ predictions for our four fits and Attila in fig. 1.2.9. It will be very interesting to see how the E814 data compare to these prediciions, since for $5^{\circ}<\theta<15^{\circ} d E / d \theta$ is sensitive to the differences in the : ojectile region between the Attila model and our model independent fits.

It should be emphasized that the four fits are conservative in that each assumes that all of the E802 spectrometer data are systematically low by at least $30 \%$. There are, of course, other less transparent solutions which are consistent both with the spectrometer data and with conservation laws. For example, abnormally large numbers of $\pi^{0}$ s, photons, or high-energy electrons could be produced in these collisions without being detected by the spectrometer. These solutions, however, imply bizarre and unprecedented physics. The four fits discissed above are thus the least unusual solutions which are more or less consistent with the reported E802 spectrometer data. One might argue that simplest solution of all tu the problem of "missing" momentum is that the E802 spectrometer data are systematically low by $20-40 \%$ at low rapidities and by $40-70 \%$ at high rapidities. If that were the case, a number of conventional models would be able to reproduce both the spectrometer $d N / d y$ and the TMA $d N / d \eta$ data reasonably well. If the spectrometer data do in fact have such large systernatic errors for central $\mathrm{Si}+\mathrm{Au}$ collisions, then one might expect similar errors to be present in central $\mathrm{Si}+\mathrm{Al}$ data, where the extrapolation to projectile rapidities is more accurately known from the approximate symmetry of the projectile and target. However, in his $\mathrm{Ph} . \mathrm{D}$. thesis, Matt Bloomer performed an analysis using symmetric functions in which he found that energy conservation together with ZCAL data insplied that the total systematic errors of the spectrometer data were less than $20 \%$ for central $\mathrm{Si}+\mathrm{Al}$ collisions[Bloo]. We are led to conclude either that new systematic errors are present in central $\mathrm{Si}+\mathrm{Au}$ collisions or that some new and unexpected physics occurs (i.e. anomalousiy large neuiral paricle production, or large numiners of fiñal particles in the projectile region). 
For the remainder of this paper, we take the normalization of the E802 spectrometer data at face value, ignoring the E810 and E814 data. None of the models which we have considered in this paper are consistent with the normalization of the spectrometer data; therefore, those models cannot be used to assess the stopping power impli $d$ by these data. For that purpose we now construct hybrid models that can reproduce the reported E802 data. It should be emphasized that these models will not be able to simultaneously reproduce high rapidity E810 and E814 data for reasons of momentum conservation as demonstrated above.

\subsubsection{Hybrid Models}

The most straight-forward way to generalize the firestreak model to incorporate transparency is to assume that each tube-tube interaction produces two firestreaks (projectile and target) rather than one. We must then determine the rapidity $\left(y_{i}\right)$ and rert eneigy per baryon $\left(M_{i}^{*}\right)$ for each of these streaks. In order to treat projectile and target consistently, we must either pick $y_{p}$ and $y_{t}$ or $M_{p}^{*}$ and $M_{t}^{*}$, since the remaining two can be solved for by energy and momentum conservation. A simple linear parametrization of the projectile and target streak rapidities is given by

$$
y_{p}=y_{p 0}-\left(\frac{N_{t}}{N_{0}}\right)\left(\frac{\sigma_{i n}}{a_{\perp}}\right) \text { and } y_{t}=\left(\frac{N_{p}}{N_{0}}\right)\left(\frac{\sigma_{i n}}{a_{\perp}}\right)
$$

where $N_{0}$ is the number of nucleons in a tube of size $a_{\perp}=\sigma_{i n}=30 \mathrm{mb}$ necessary to cause a one unit rapidity shift of the opposing tube. The last factor in each of the above equations was included to insure that the stopping power would be independent of the lattice size $\left(a_{\perp}\right)$ chosen. Unfortunately, the above prescription leads to a number of cases where $M^{*}$ of one of the fireballs is forced by 4-momentum conservation to be less than the mass of the nucleon. fig. 1.2 .10 shows the regions of $\left(N_{p}, N_{t}\right)$ space for which this problem arises. Similar problems were encountered with other parametrizations in which $y_{p}$ and $y_{t}$ were chosen independentily.

These problem regimes could in principle be handled specially by demanding com- 
plete transparency or the formation of a single fireball, but we chose instead to utilize a different algorithm which avoids special cases. First, in the center-of-mass frame of two colliding tubes containing $N_{p}$ and $N_{t}$ nucleons, the incoming momentum, $P^{*}$, is

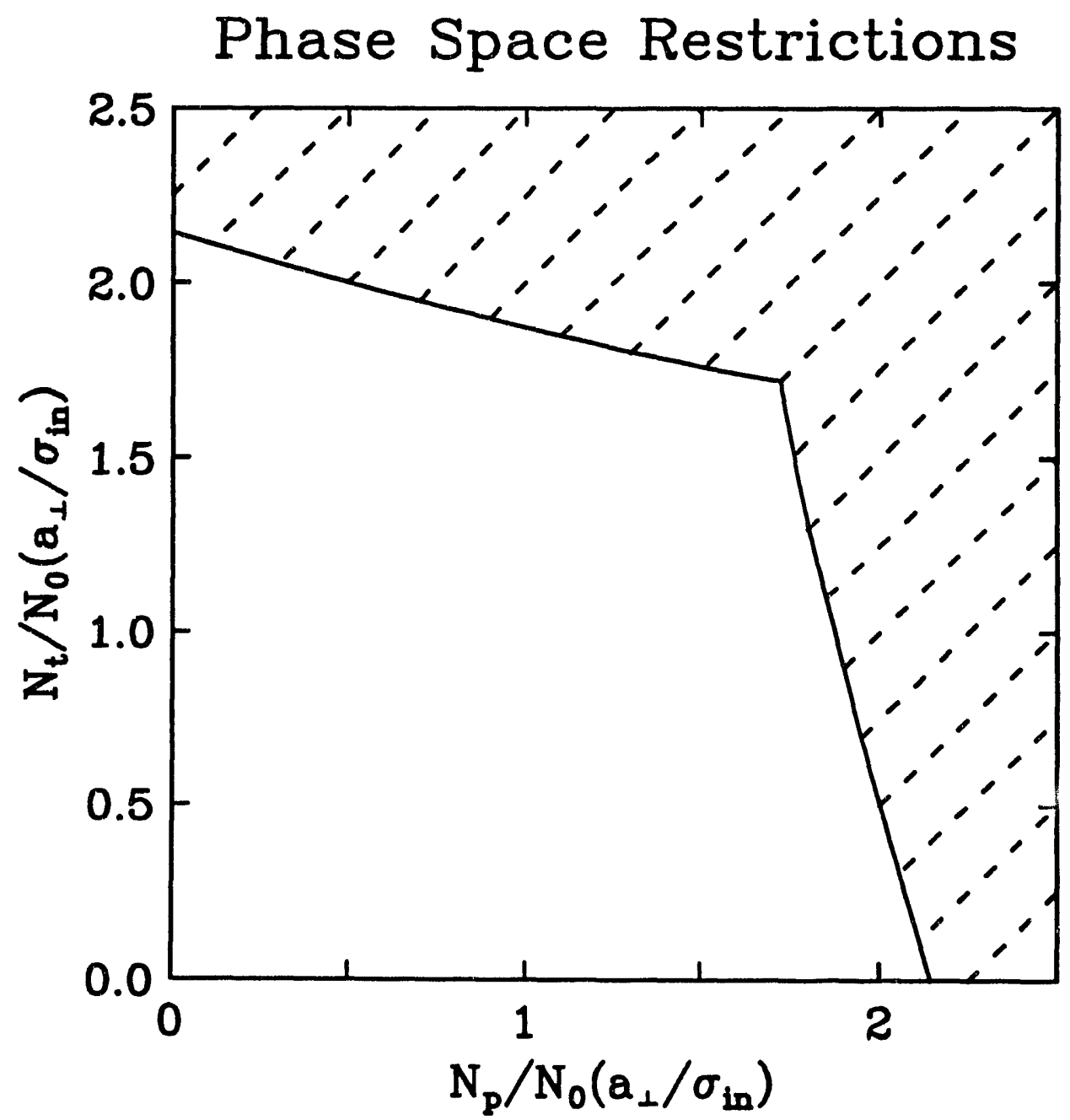

Figure 1.2.10: The available phase space for the stopping prescription of eqn. (15) is shown by the unshaded region. In the shaded region, one or both of the receding fireballs must have a mass/baryon $<.939 \mathrm{GeV}$ in order to conserve 4-momentum.

found. Next, the momentum of each tube is reduced by an amount proportional to the number of binary collisions, $N_{p} N_{t}$ :

$$
\Delta P^{*}=\delta p_{z} N_{p} N_{t}\left(\frac{\sigma_{\text {in }}}{a_{\perp}}\right)
$$

Finally, the energy/baryon is required to be the same for both of the outgoing fire- 
streaks $\left(M_{p}^{*}=M_{t}^{*}=M^{*}\right) . M^{*}$ and the $\mathrm{CM}$ firestreak rapidities $y_{p}^{*}$ and $y_{t}^{*}$ can then be found from the following equations:

$$
\begin{gathered}
M^{*} N_{p} \sinh \left(y_{p}^{*}\right)=M^{*} N_{t} \sinh \left(y_{t}^{*}\right)=P^{*}-\Delta P^{*} \\
M^{*} N_{p} \cosh \left(y_{p}^{*}\right)+M^{*} N_{t} \cosh \left(y_{t}^{*}\right)=M_{c m}\left(N_{p}+N_{t}\right),
\end{gathered}
$$

where the CM energy/baryon of the tube-tube system, $M_{c m}$, is determined by kinematics. Due to the symmetries of this method, $M^{*}$ monatonically increases from $m_{N}$ to $M_{c m}$ as $\Delta P^{*}$ is increased from 0 to $P^{*}$. When the prescription of (16) gives a $\Delta P^{*} \geq P^{*}$, a single firestreak with $M^{*}=M_{c m}$ and $y_{p}^{*}=y_{t}^{*}=0$ is assumed to be formed. Defining the effective nuclear thickness, $z_{i}$, via $N_{i}=a_{\perp} \rho_{0} z_{i}$, the momentum shift per baryon of the projectile (target) is thus assumed to increase linearly with the effective target (projectile) thickness. The nuclear stopping power of this model is controlled by a single parameter-the momentum loss per binary collision $\delta p_{z}$, or equivalently, the nuclear stopping length

$$
L_{s}=m_{N} \sinh \left(y_{p 0} / 2\right) /\left(\sigma_{i n} \rho_{0} \delta p_{z}\right)
$$

The meaning of this stopping length can be most easily seen in symmetric collisions $\left(z_{p}=z_{t}=z\right)$, where the fractional momentum loss $\left(\Delta P^{*} / P^{*}=z / L_{s}\right)$ increases linearly and reaches unity when $z=L_{s}$. Thus a stopping length of $10 \mathrm{fm}$ implies that two colliding tubes of length $10 \mathrm{fm}$ will just be able to stop each other.

In fig. 1.2.11 we compare models with various values of $L_{s}$ to the data $\left(\rho_{f_{r}}=2 \rho_{0}\right.$ and $\gamma_{s}=0.7$ have been chosen to provide the best agreement with kaon data and pion temperatures). Compared to the data, $L_{s}=10 \mathrm{fm}$ is evidently too small and $L_{s}=26 \mathrm{fm}$ is too large. Though $L_{s}=17 \mathrm{fm}$ provides good agreement to all but the last point of the $d N_{p} / d y$ data, its pion peak is shifted to low rapidities, and its proton temperature is too low with a dip at midrapidity which is not seen in preliminary, unpublished $T_{p}(y)$ data[Bloo]. It should be noted that folding the E802 spectrometer acceptance[E802a] into the double firestreak leads to less than a $10 \%$ suppression 


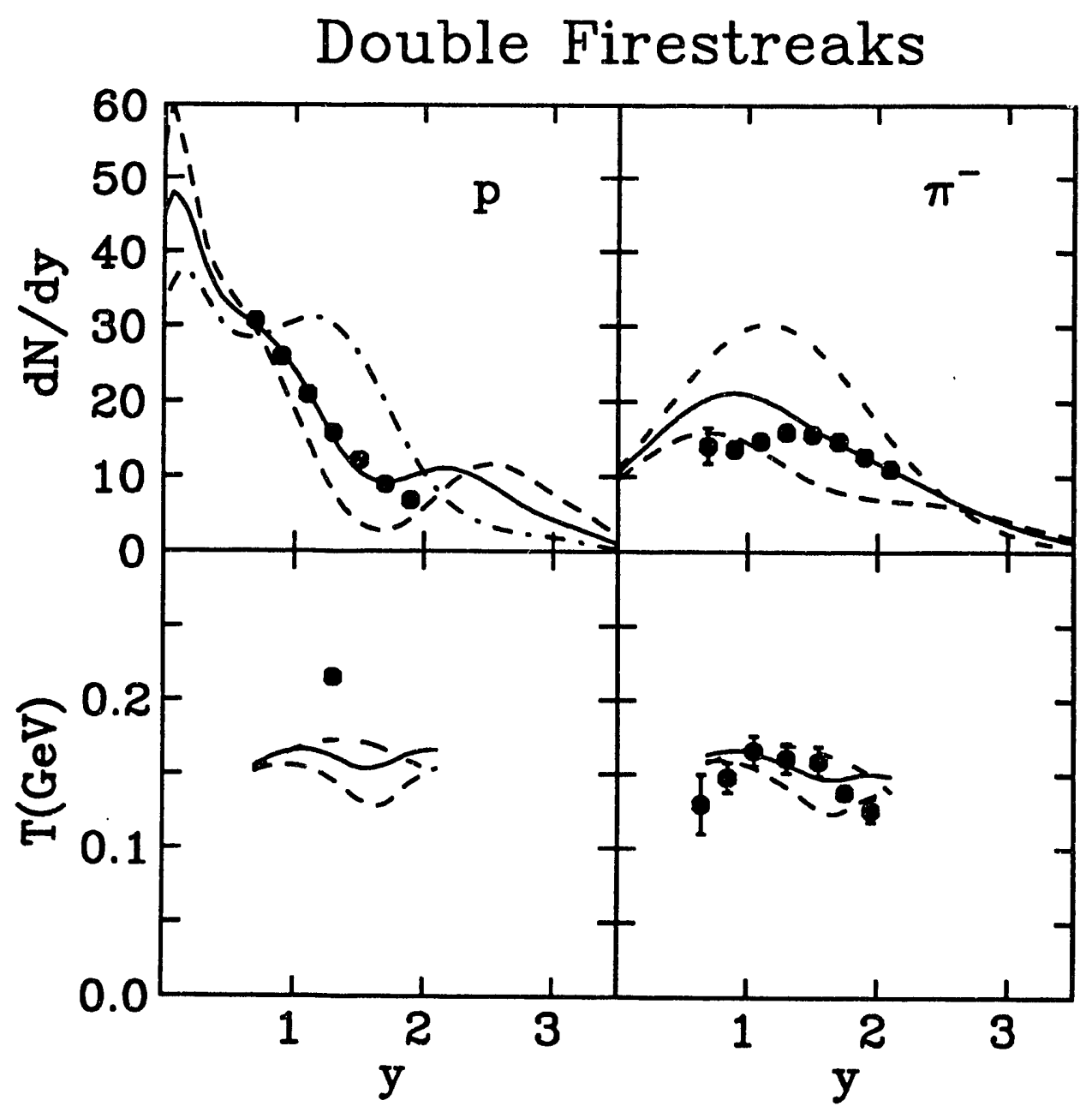

Figure 1.2.11: Double firestreaks with $L_{s}=10 \mathrm{fm}$ (dot-dashed), $17 \mathrm{fm}$ (solid), and 26 fm (dashed) are compared the data of fig. 1.2.2.

of the pion yield and no discernable change in the proton rapidity spectrum. This double firestreak description provides far better agreement with the data than any of the other models discussed so far, but in order to quantitatively reproduce all the features of the E802 data, further refinements are needed.

One of the key observations of E802 is that the transverse momentum slopes of protons and pions differ significantly. Therefore the amount of energy locked into transverse motion differs from that expected in simple thermal models with one freezeout temperature. Collective flow[Brown] provides one natural mechanism for different slopes. Different freezeout criteria due to different cross sections provides another. To 
test the effect of this difference on the conclusion of the stopping power, we developed a more complex multicomponent model $(\mathrm{mcm})$. The details of this model are given in the Appendix. We emphasize that the $\mathrm{mcm}$ is not meant to be a realistic model of the physics, but a convenient numerical tool to help sort the implications of various features of the data.

In figs. 1.2.12 and 1.2.13 we show two mcm solutions. The solid line is the best fit to the data (mcm1), with $L_{s}=L_{s}^{\prime}=26 f m, M_{1}^{*}=1.4 \mathrm{GeV}, M_{2}^{*}=1.85 \mathrm{GeV}, \rho_{f r}=\rho_{0}$, $T_{\text {mes }}=160 \mathrm{MeV}$, and $\gamma_{s}=0.25$. This is the fit that was used by us in ref.[Chap91].

\section{Multicomponent Model Fits}

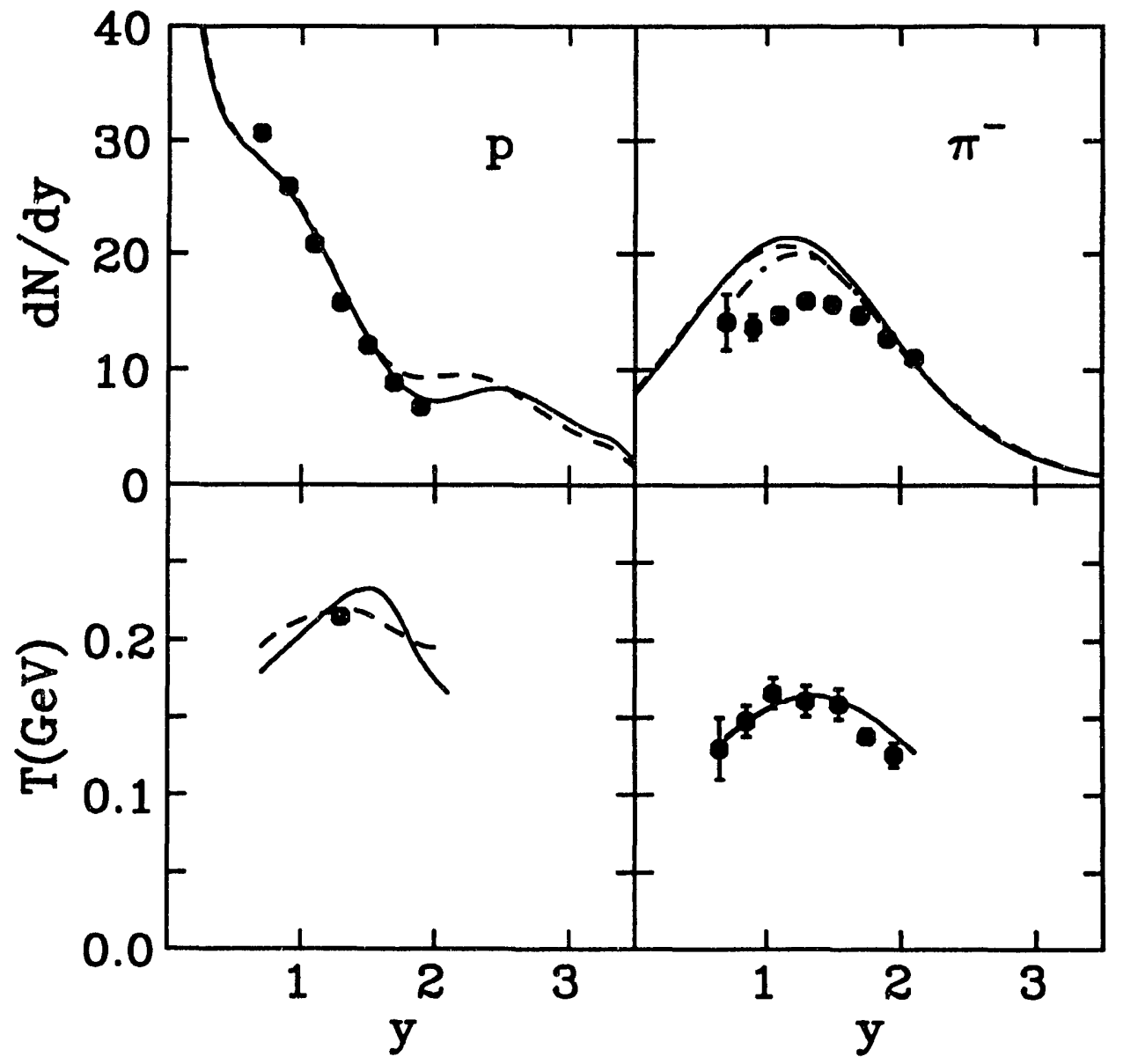

Figure 1.2.12: Multicomponent model fits mcm1 (solid) and mcm2 (dashed) are compared to the data of fig. 1.2.2. The dot-dashed curve for the pions shows the result of $\mathrm{mcm} 1$ with the experimental acceptance taken into account[E802a]. 

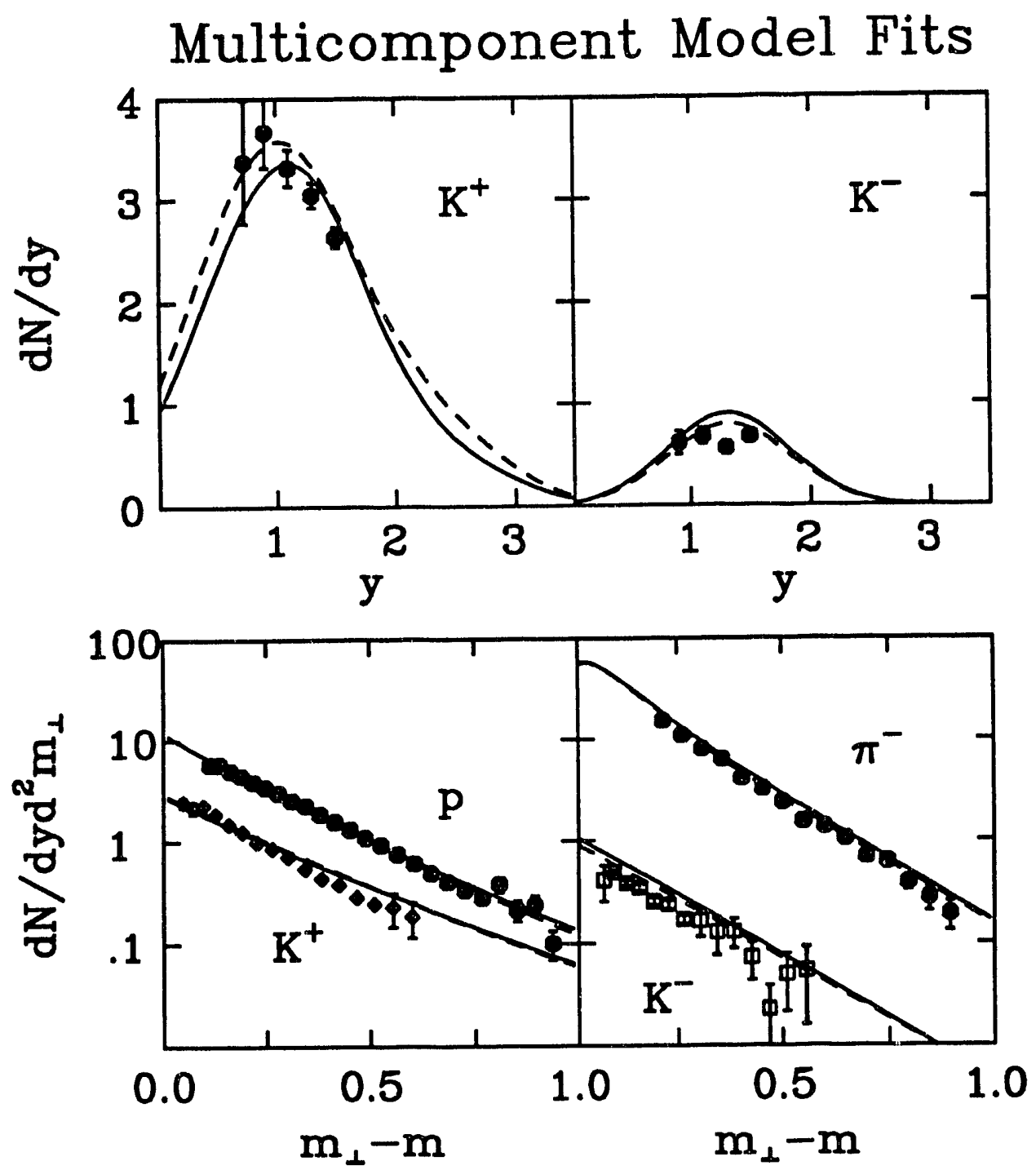

Figure 1.2.13: Multicomponent model fits $\mathrm{mcm} 1$ (solid) and $\mathrm{mcm} 2$ (dashed) are compared to the data of fig. 1.2.6.

The dashed curve is another fit to the data $(\mathrm{mcm} 2)$ with $L_{s}=20 \mathrm{fm}, L_{s}^{\prime}=50 \mathrm{fm}$, $M_{1}^{*}=1.55 \mathrm{GeV}, M_{2}^{*}=2 \mathrm{GeV}, \rho_{f r}=\rho_{0}, T_{\text {mes }}=165 \mathrm{MeV}$, and $\gamma_{s}=0.25$. The dot-dashed curve for the pions shows $\mathrm{mcm} 1$ with the spectrometer acceptance[E802a] folded in. Due to the many adjustable parameters of this model, both mcml and $\mathrm{mcm} 2$ are able to quantitatively reproduce almost all of the E802 spectrometer data. The most notable discrepancy is the $25 \%$ overprediction of low rapidity pions by these models after the experimental acceptance has been taken into account. Unlike the 
models discussed previously which also overpredict pions, the disagreement of the mom fits is much smaller and only seen at low rapidity. Both of the $\mathrm{man}$ fits as well as the $L_{s}=17$ double firestreak exhibit the high degree of nuclear transparency necessary to be able to reproduce the $\mathrm{E} 802$ spectrometer data.

The $L_{s}=17$ double firestreak as well as $\mathrm{mcm} 1$ and $\mathrm{mcm} 2$ discussed above have all had their parameters tuned to best fit the $\mathrm{E} 802$ central $\mathrm{Si}+\mathrm{Au}$ spectrometer data. The quality of these fits is therefore not very surprising, especially in the case of the $\mathrm{mcm}$ where there are so many free parameters. An interesting test of these models is to see how well they can reproduce unpublished E802 central $\mathrm{Si}+\mathrm{Al}$ and $\mathrm{Si}+\mathrm{Cu}$ data[Bloo]. For these reactions, there is very little difference between the results of $\mathrm{mcm} 1$ and $\mathrm{mcm} 2$; both of them are able to reproduce $d N_{\pi} / d y$ and mid-rapidity $d N_{p} / d y$ of both collisions to within $20 \%$. Both parameter sets predict too many target protons, but this could be due to large fragment formation in these reactions. The $L_{s}=17$ double firestreak obtains results similar to $\mathrm{mcm} 1$ and $\mathrm{mcm} 2$ for $\mathrm{Si}+\mathrm{Cu}$, but it exhibits a factor of 2 too few mid-rapidity protons and pions in central $\mathrm{Si}+\mathrm{Al}$ collisions. Even though the double firestreak uses a smaller value for $L_{0}$ than the mom fits, it exhibits less stopping when applied to lighter nuclei. This is because there is no center-of-mass firestreak in the double firestreak model, so a lot of energy is carried away by receding mesons. This effect becomes much more pronounced with less stopping (lighter nuclei). It should be noted that we were not able to find a model which could simultaneously fit E802 p+A data and central A+B data. However, to the extent that central $A u+A u$ reactions bear more similarity to central $\mathrm{Si}+\mathrm{A}$ than to $\mathrm{p}+\mathrm{A}$ reactions, the predictions for $\mathrm{Au}+\mathrm{Au}$ by our $\mathrm{mcm}$ fits are better supported by the E802 spectrometer data than those of the models discussed in the first parts of this paper. In figure 1.2.14 we show $\mathrm{Au}+\mathrm{Au}$ predictions by the $L_{s}=10$ fm (dot-dot-dashed) and $L_{s}=17 \mathrm{fm}$ (dot-dashed) double firestreaks as wel! as by the $\mathrm{mcm}$ fits $(\mathrm{mcm} 1=$ solid, $\mathrm{mcm} 2=$ short-dashed $)$. For such large nuclei, tine $L_{x}=10$ fm double firestreak forms essentially a fully-stopped firestreak which consequently 
features a much narrower and higher peak in $d N_{p} / d y$ than the other models. This is due to the fact that full stopping has not been achieved in these models, as can be easily seen by looking at the long dashed line which represents the projectile proton rapidity distribution of $\mathrm{mcm} 1$. Since $\mathrm{Au}+\mathrm{Au}$ is symmetric, the projectile and target contributions combine to form a. symmetric, gaussian-like $d N_{p} / d y$ which would be difficult to differentiate from the result that one would get from a fully-stopped fireball undergoing longitudinal expansion. For asymmetric collisions like $\mathrm{Si}+\mathrm{Au}$, on the other hand, these two cases can be clearly distinguished. For this reason it is important to study and understand asymmetric as well as symmetric collisions.

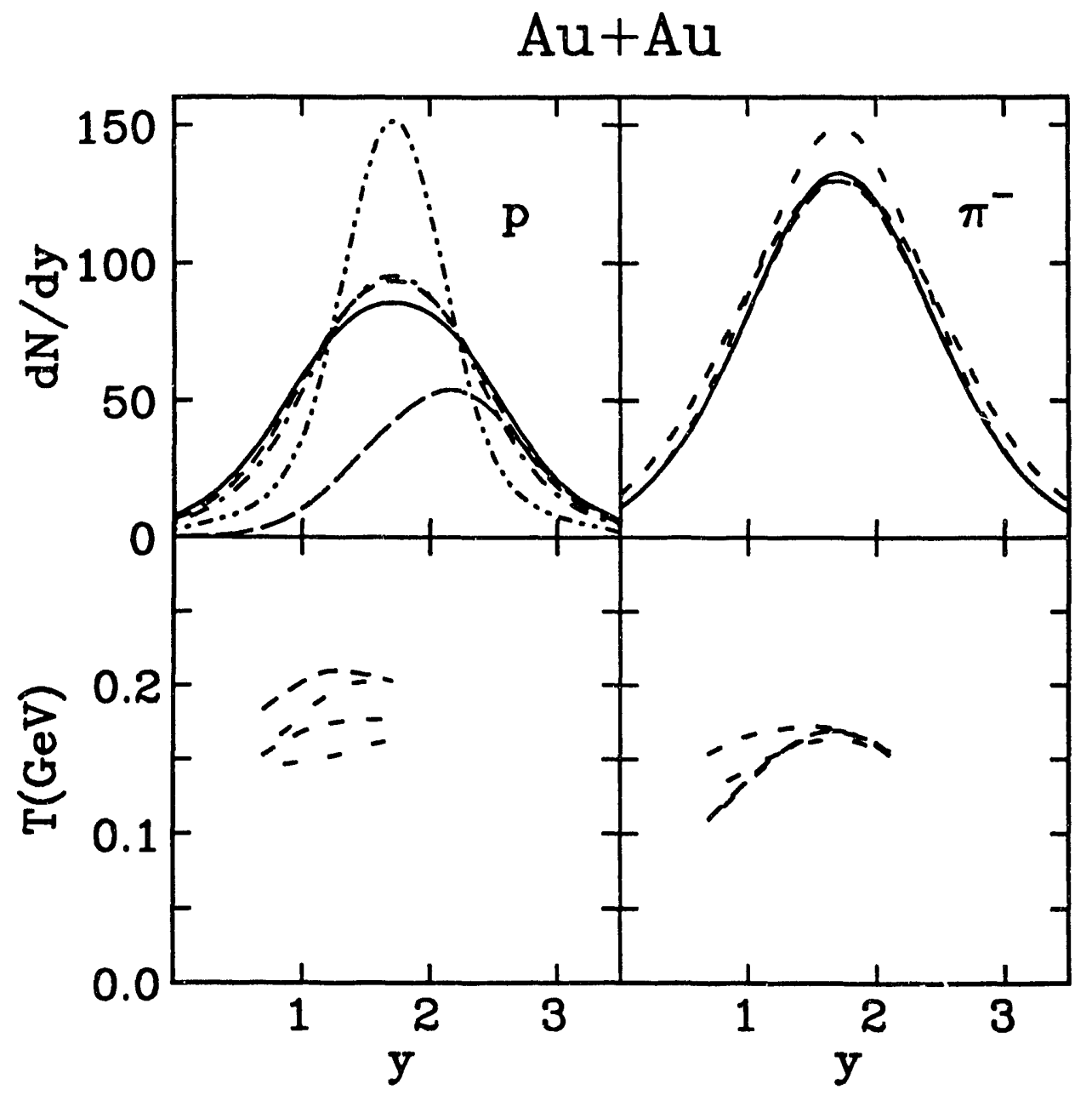

Figure 1.2.14: Predictions for central $(0<b<3 \mathrm{fm}) \mathrm{Au}+\mathrm{Au}$ collisions by multicomponent model fits $\mathrm{mcm} 1$ (solid), $\mathrm{mcm} 2$ (dashed), and double firestreaks with $L_{\mathbf{s}}=10$ $\mathrm{fm}$ (dot-dot-dashed) and $L_{s}=17 \mathrm{fm}$ (dot-dashed). Projectile protons for $\mathrm{mcm} 1$ are shown by the long-dashed curve in the upper left panel. 


\subsubsection{Conclusion}

We showed that none of the present models which assume complete nuclear stopping and none of the present nonequilibrium string models are consistent with the published E802 spectrometer data[E802] for central $\mathrm{Si}+\mathrm{Au}$ reactions. For example, even the RQMD model is not consistent with these data, as they note that "the problem of the 'missing' energy-momentum could be resolved if the normalization of the E802 spectrometer data were too small."[RQMD92] If corrections to the normalization nowhere exceed $30 \%$, then energy-momentum and baryon conservation alone require the existence of at least $10-11$ nucleons in the projectile region $(y>2.44)$ which, however, would be inconsistent with E810[E810] and E814[E814] results. The fact that the high rapidity E810 and E814 proton data are in excellent agreement, even though the E810 trigger is less central and the E814 trigger is more central than E802, makes it unlikely that the discrepancies of those data with fits to the E802 spectrometer are due to triggering effects alone. A double firestreak and a multicomponent model have been developed to quantify the degree of transparency needed to reproduce the spectrometer data, and nuclear stopping lengths of 17-26 fm were found. These lengths are much larger than the lengths of 8-10 fm which were expected based on other experiments at these and higher energies[pA]. On the other hand, the high rapidity data from E810 and E814 as well as preliminary $d N / d \eta$ data from E802[HIPAGS] and E814[E814a] are consistent with models incorporating the expected degree of nuclear stopping. Until the discrepancies between all data sets are resolved, conclusions about full nuclear stopping remain premature. 


\subsubsection{Appendix}

In the multicomponent model, we decompose a single fireball into two with two different freezeout times (one baryonic and one mesonic). Baryonic fireballs are assumed to consist of baryons (no antibaryons), $K^{+}$'s and $K^{0}$ 's balanced such that they have zero net strangeness. Since the baryon resonances are allowed to decay as usual, there are some pions which are produced by baryonic fireballs. Mesonic fireballs are comprised of all hadronic resonances (including baryons), but have zero baryon number and strangeness. We suppose that each tube-tube collision gives rise to one fully stopped, double-freezeout firestreak at the local center of mass as well as to receding projectile and target baryonic firestreaks. A number of new parameters must be introduced into this model to determine the energy and baryon number of each of the firestreaks involved.

First, as in the double firestreak, a value of $L_{s}$ is specified in order to determine $M^{*}, y_{p}^{*}$, and $y_{t}^{*}$ for the receding firestreaks. Second, another stopping length, $L_{s}^{\prime}$, is chosen in order to determine the fraction of baryons from each tube which get fully stopped:

$$
f_{s}=\left(z_{p} z_{t}\right)^{1 / 2} / L_{s}^{\prime} .
$$

Next, if the initial CM energy/baryon, $M_{c m}$, of the tube-tube system is greater than an excitation mass parameter $M_{2}^{*}$, then the energy/baryon of the baryonic part of the central fireball is limited to $M_{c}^{*}=M_{2}^{*}$, and the energy/baryon available to the receding streaks becomes

$$
M^{*} \rightarrow M^{* \prime}=\frac{1-f_{s}\left(M_{2}^{*} / M_{c m}\right)}{1-f_{s}} M^{*}
$$

in order to conserve energy. If, on the other hand, $M_{c m} \leq M_{2}^{*}$, then $M_{c}^{*}=M_{c m}$ and $M^{* \prime}=M^{*}$. If $M^{* \prime}$ turns out to be smaller than another parameter $M_{1}^{*}$, then there is no mesonic firestreak at all, and the tube-tube interaction is modeled by three purely baryonic streaks. However, if $M^{* \prime}>M_{1}^{*}$ then the receding streaks have their energy/baryon limited to $M_{1}^{*}\left(M_{p}^{*}=M_{t}^{*}=M_{1}^{*}\right)$, and a mesonic streak overlapping 
the CM baryonic streak is created with energy

$$
E_{m e s}=\left(M^{* \prime}-M_{1}^{*}\right)\left(1-f_{s}\right)\left(N_{p} \cosh \left(y_{p}^{*}\right)+N_{t} \cosh \left(y_{t}^{*}\right)\right)
$$

For the mesonic streaks, a freezeout temperature $T_{m e s}$ is specified and $V_{f r}$ is solved for trivially, since $\mu=\mu_{s}=0$ for streaks with zero baryon number and strangeness. Note that if $\left(z_{p} z_{t}\right)^{1 / 2} \geq L_{s}^{\prime}$ or $\Delta P^{*} \geq P^{*}\left(\left(z_{p} z_{t}\right)^{1 / 2} \gtrsim L_{s}\right)$ for any two incoming tubes, then this model reduces to a fully-stopped firestreak with separate baryonic and mesonic freezeout criteria.

The many parameters of this model have interrelated effects but can be approximately explained as follows. The amount of baryon stopping is controlled by $L_{s}$ and $L_{s}^{\prime}$. The central $(1.1<y<1.7)$ values of $T_{p}(y)$ are controlled by $L_{s}^{\prime}, M_{2}^{*}$, and $\rho_{f r}$, while the wings $(y<1.1, y>1.7)$ of $T_{p}(y)$ are controlled by $M_{1}^{*}$ and $\rho_{f r}$. It should be noted that for baryonic firestreaks with $M_{i}^{*}$ fixed, decreasin $n_{f r}$ cools the baryons by forcing them into higher mass resonances. $T_{\pi}(y)$ is mainly controlled by $T_{\text {mes }}$, though $\rho_{f r}, M_{1}^{*}$ and $M_{2}^{*}$ also have effects by adjusting the number of cool pions coming from baryon resonances. The height of $d N_{\pi} / d y$ is affected by all of the parameters; increasing the value of any one of them leads to a decrease in the number of pions. The overall number of kaons is adjusted by $\gamma_{s}$, while the $K^{+} / K^{-}$ratio is determined by the number of strange baryons, which is again a function of $\rho_{f r}, M_{1}^{*}$ and $M_{2}^{*}$. 


\title{
1.3 Talk from Quark Matter '91
}

by Scott Chapman

(Published in Nuclear Physics A544 (1992) p. 429)

\begin{abstract}
Fireball, firestreak and hadronic string models are shown to overpredict recent central $15 \mathrm{AGeV} \mathrm{Si}+\mathrm{Au}$ E802 spectrometer data by at least $70 \%$. Claims in the literature about full nuclear stopping in $\mathrm{Si}+\mathrm{Au}$ reactions are therefore premature. In fact, fits to the spectrometer data indicate that up to half of the projectile nucleons may lose less than one unit of rapidity after traversing 5-10 fm of nuclear matter, implying possibly a surprisingly long stopping length of $\sim 20 \mathrm{fm}$. Comparison of these same fits with E810, E814, and preliminary E802 $d N_{\text {charged }} / d \eta$ data suggests, however, that there may be some inconsistencies among the various data sets, and therefore that additional data will be needed to establish the degree of nuclear stopping at AGS energies.
\end{abstract}

\subsubsection{Introduction}

It is popularly believed that at the AGS "full stopping is realized[PANIC], showing a behavior close to the Landau model[Stach] and to relativistic fluid dynamics[Stau89], and the energy density can reach values comparable to the critical values for QGP formation"[Ame91]. However, as we pointed out in refs. [Chap91, Chap92], the published E802 spectrometer data[E802] cast doubt on this belief, since in fact none of the present models is consistent with the full array of data. Moreover, unless the systematic errors of the spectrometer data are very large, these data are more indicative of a surprising degree of nuclear transparency. As we show below, however, no firm conclusion can be made on this important topic, since not all of the data sets are completely consistent. In this paper our aim is to clarify what are the problems at present in drawing conclusions about nuclear stopping power in these reactions.

In our letter[Chap91] we discussed a model independent fit to the spectrometer data which implied that if systematic errors do not cause more than a $30 \%$ suppression of proton and pion yields, then 4-momentum and baryon conservation laws imply that at least 11 out of 28 projectile nucleons suffer less than one unit of rapid- 
ity loss during the collision. In our subsequent paper[Chap92], we gave the precise functional form of the fit used in the letter, and introduced three other fits which featured unexpectedly large $n / p$ ratios in the projectile region. Here we introduce a fifth 4-momentum-conserving fit which features a more realistic $\mathrm{n} / \mathrm{p}$ ratio and can successfully reproduce the E810[E810] data and extrapolations of E814[E814] leading neutron data (errors are estimated by assuming . $1 \mathrm{GeV}<\mathrm{T}<.2 \mathrm{GeV}$ ), but which overpredicts the currently published E802 spectrometer data by $40 \%-70 \%$, while still underpredicting preliminary E802 $d N_{\text {charged }} / d \eta$ data[HIPAGS]. We conclude that unlike E810, E814 and E802 $d N_{\text {charged }} / d \eta$ data, the E802 spectrometer data do not support the claims of full nuclear stopping which are so prevalent in the literature[PANIC, Stach, Ame91, RQMD91, RQMDpi, Brown, BrownEr].

\subsubsection{Comparison of Models to the Data}

The solid line in fig. 1.3.1 shows the results of the generic fireball model outlined in ref. [Chap92] with $\rho_{f r}=5 \rho_{0}$ and $\gamma_{s}=.5$. This fireball model produces more than a factor of 2 too many protons, pions, and kaons (not shown) at mid-rapidity. No reasonable variation of $\rho_{f r}$ and/or $\gamma_{s}$ significantly improves agreement with the data. In addition to the generic fireball, fig. 1.3.1 also shows results from the Landau hydrodynamic longitudinally expanding fireball[Stach] (dashed line) and the hydrochemical spherically expanding fireball[Brown, BrownEr] (dot-dashed line). The longitudinal expansion of the Landau fireball results in reduced proton and pion peaks at midrapidity. This expansion, however, only shifts the problem to higher rapidities, where again the model produces a factor of 2 more protons than are seen in the data. Even though the spherical expansion of the hydrochemical model provides a possible explanation for the difference in proton and pion slopes, the model again fails to reproduce the measured norms of these distributions. In fact, all of the fireball models considered here overpredict the measured proton and/or pion rapidity distributions by about a factor of 2 . 


\section{Fireballs}
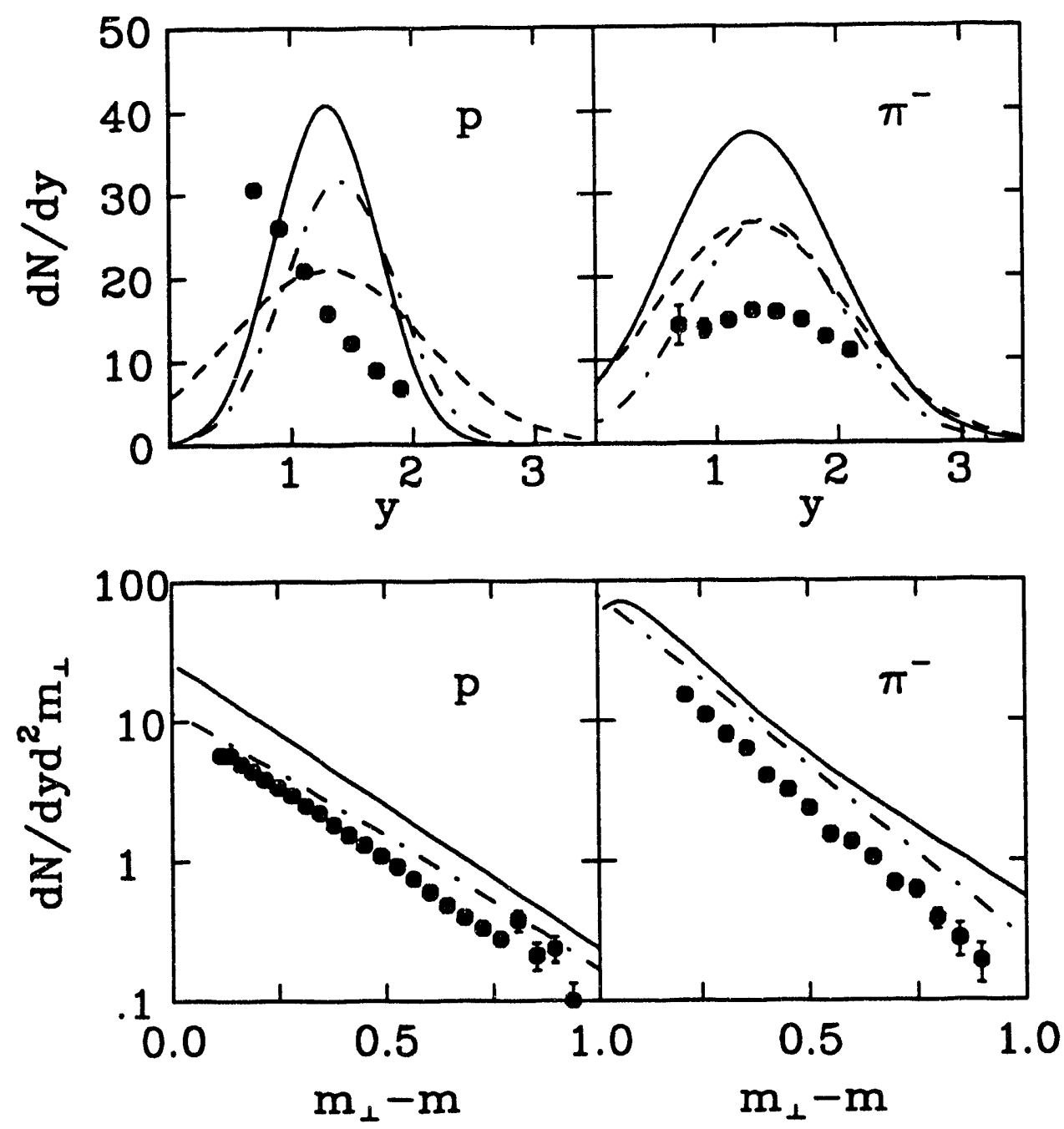

Figure 1.3.1. Landau hydrodynamic[Stach], hydrochemical[Brown, BrownEr], and generic[Chap92] fireballs are compared to E802 proton and pion spectrometer data from 14.6 AGeV/c central $S i+A u$ reactions[E802]. The bottom panels are for $y=1.3$.

In fig. 1.3.2, we compare the firestreak[Chap91, Chap92] and two string models (Attila[Gyu87] and RQMD[RQMD91, RQMDpi]) with the data. For the Firestreak and Attila, we have calculated $T_{i}(y)$ by fitting the invariant distributions with exponentials

$$
d N_{i} / d y d^{2} m_{\perp}=\rho_{i}(y) \exp \left(-\left(m_{i \perp}-m_{i}\right) / T_{i}(y)\right)
$$

in order to compare our curves to the published $T_{i}(y)$ values. Though the firestreak improves on the fireball by showing "spectator" contributions, it still predicts a factor 
of 2 too many mid-rapidity protons and pions. The string models do a better job of reproducing the overall ramp shape of $d N_{p} / d y$, though they overpredict the number of high rapidity protons by $50 \%$. As for the pions, the string models again do better than the firestreak, though they still overpredict by $70 \%$ the $d N_{\pi} / d y$ values reported by E802. Comparing Attila to RQMD shows that rescattering does not significantly improve the string model fits to the rapidity data. It should also be noted that the quark-gluon string model recently proposed in ref.[Ame91] similarly overpredicts the number of mid-rapidity pions by at least $70 \%$.

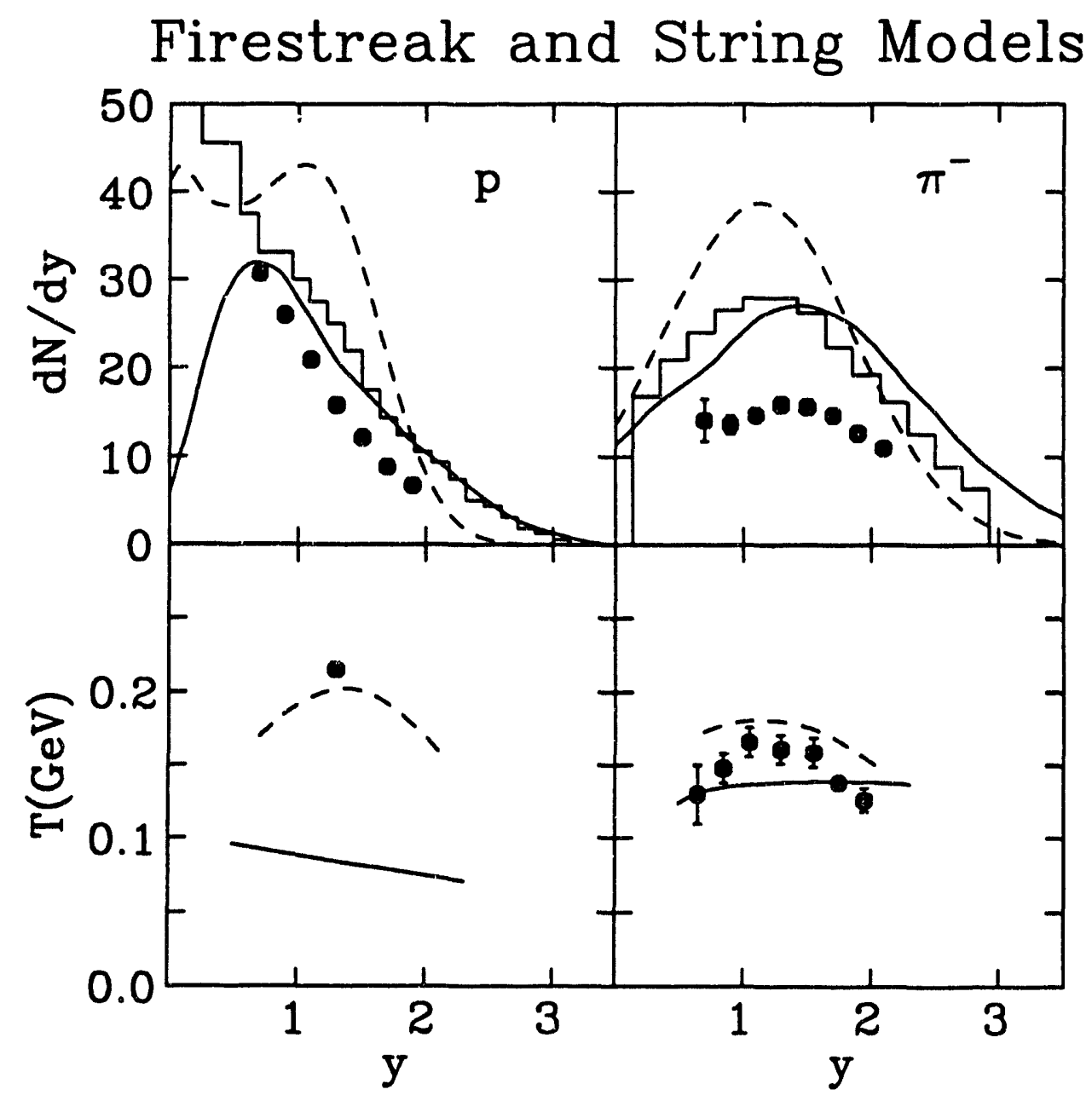

Figure 1.3.2. Firestreak[Chap91, Chap92] (dashed), Attila[Gyu87] (solid), and RQMD [RQMD91, RQMDpi] (histogram) calculations are compared to the same data as in fig. 1.3.1. The bottom panels are the inverse slope parameters of eqn. (1.3.1)[E802]. 


\subsubsection{Model Independent Fits}

Having seen that none of the above equilibrium and nonequilibrium models for nuclear collision dynamics are able to reproduce the published spectrometer data, we consider next a model independent fitting procedure in order to isolate possible causes for the discrepancies. As in ref. [Chap92], we begin by fitting the experimental $T_{i}(y)$ [Bloo] and $(d N / d y)_{i}(y)[E 802]$ data with simple functions which have reasonable extrapolations to phase space regions outside of the experimental acceptance (dot-dashed line of fig. 1.3.3). The invariant distributions, $f_{i}=d N_{i} / d y d^{2} m_{\perp}$, are then completely determined if the exponential form of eqn. (1) is assumed, since

$$
d N_{i} / d y=2 \pi \rho_{i}(y) T_{i}(y)\left(T_{i}(y)+m_{i}\right)
$$

The exact functional forms of the fits that we used (for kaons as well) are given in ref. [Chap92]. For the unobserved neutral mesons it is assumed that $\pi^{0}=\left(\pi^{+}+\pi^{-}\right) / 2$, $K^{0}=K^{+}$, and $\bar{K}^{0}=K^{-}$. Charge conservation is enforced by demanding that the total number of final protons be $N_{p}=14+79-N_{\pi^{+}}-N_{K^{+}}+N_{\pi^{-}}+N_{K^{-}}$. With $N_{p}$ fixed, the total number of undetected neutrons is given by baryon number conservation, $N_{n}=28+197-N_{p}$. These fits allow us to take into account all of the observed energy in longitudinal and transverse motion as well as pion and kaon production.

The total outgoing longitudinal momentum $P_{z}$ implied by these fits is easily calculated by integrating $m_{\perp} \sinh (y) f_{i}$ over $d^{2} m_{\perp}$ and $y$ :

$$
P_{z}=\sum_{i=\text { hadrons }} \int d y \frac{2 T_{i}^{2}+2 T_{i} m_{i}+m_{i}^{2}}{T_{i}+m_{i}}(d N / d y)_{i} \sinh (y) .
$$

$\mathrm{E}$ is simply found by replacing $\sinh (\mathrm{y})$ by $\cosh (\mathrm{y})$. For the fit to the data shown by the dot-dashed lines in fig. 1.3.3, the integration over y gives $P_{z}=241 \mathrm{GeV} / \mathrm{c}$ and $\mathrm{E}=455 \mathrm{G} \cdot \mathrm{V}$, whereas the total incoming energy and momentum are known to be $P_{z}=409 \mathrm{GeV} / \mathrm{c}(=28 \times 14.6)$ and $\mathrm{E}=505 \mathrm{GeV}(=197 \times 939+28 \times 14.63)$. More than a third of the incoming momentum and a fourth of the energy are unaccounted 
for in this fit to the data! If we assume that neither leptons nor photons carry a significant fraction of the 4-momentum, then there must be some undetected hadrons somewhere which do carry it. The E802 collaboration has acknowledged that an undetected excess of low $p_{\perp}$ particles could result in a $25 \%$ normalization error of the $d N / d y$ data[E802]. To take into account these and/or other possible systematic errors in the data, we proceed by multiplying each of our $(d N / d y)_{i}$ functions by $\alpha=1.3$ and find $P_{z}=322 \mathrm{GeV} / \mathrm{c}$ and $\mathrm{E}=519 \mathrm{GeV}$. However, more than $85 \mathrm{GeV} / \mathrm{c}$ of momentum and $75 \mathrm{GeV}$ of energy are still missing!

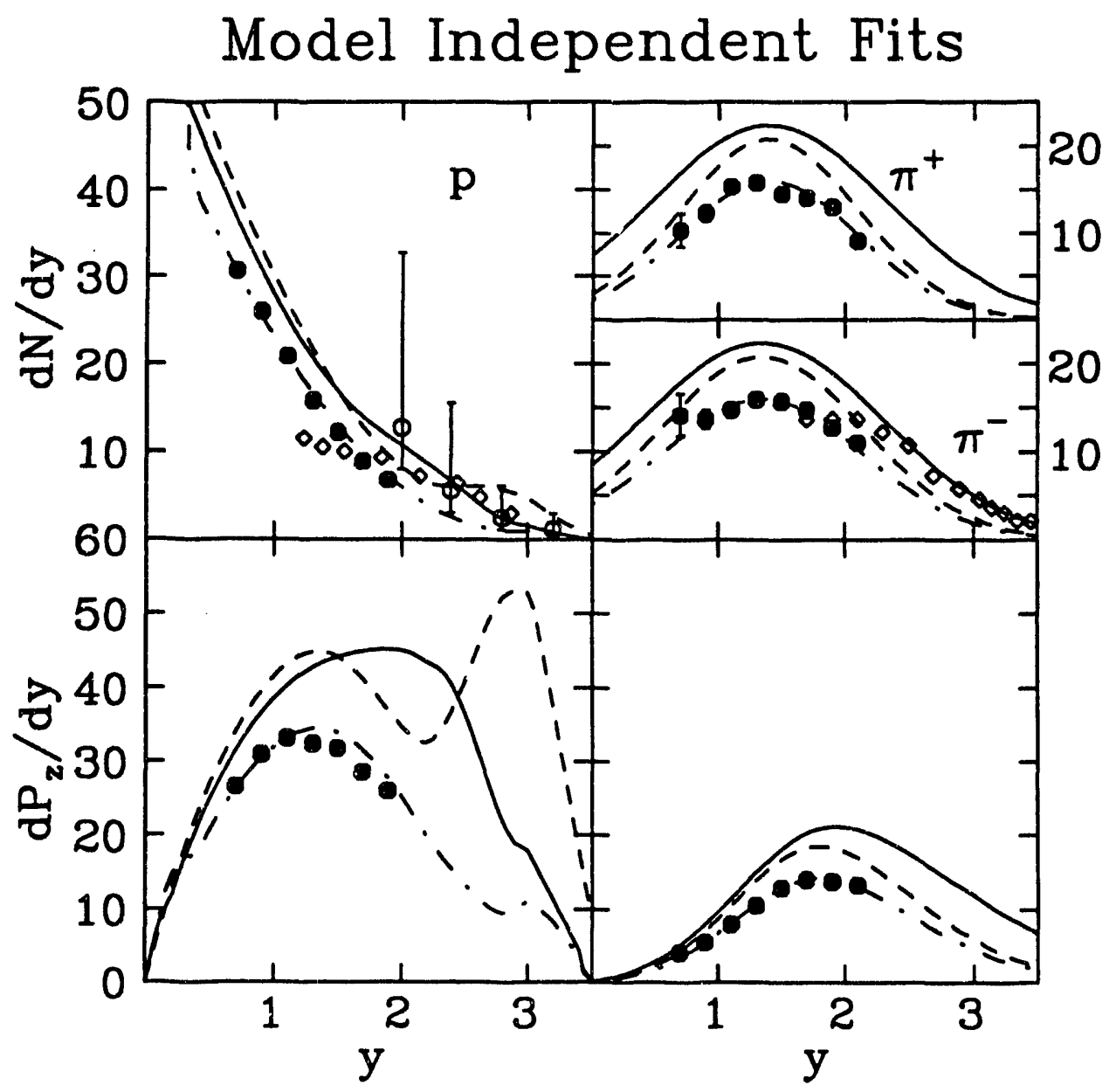

Figure 1.3.3. A fit to the data (dot-dashed), fit1 (dashed) and fit5 (solid) are compared to E802[E802] data (dots), E810[E810] negatives and $(+)-(-)$ (diamonds), and extrapolated E814[E814] protons (circles for $\mathrm{T}=.15 \mathrm{GeV}$ ).

Either the systematic errors of the $d N_{i} / d y$ data are significantly larger than $30 \%$, 
or else the "missing" 4-momentum must be carried by an unexpectedly large number of undetected high-rapidity hadrons. The least transparent solution which does not overpredict any of the data by more than $30 \%$ is given by fitl of ref. [Chap92] and is shown by the dashed lines in fig. 1.3.3. Less transparent solutions can of course be found by allowing more than a $30 \%$ discrepancy with the spectrometer data. An example of a fit of this kind is fit5 (solid lines in fig. 1.3.3), with fit parameters (see ref. [Chap92]) given by $\left(y_{\text {pro }}, C_{\text {pro }}, C_{s p e c}, \delta_{s p e c}, \delta_{\pi^{+}}, \delta_{\pi^{-}}, \alpha_{\text {mes }}, \alpha_{p}, \delta_{\text {pro }(y<2.25)}, \delta_{\text {pro(y>2.25) }}\right)$ $=(2.25,3.3,93.39,0.025,1.75,1.85,1.4,1.2,0.5,0.25)$. Fit5 has an $\mathrm{n} / \mathrm{p}$ ratio of 1.3

\section{Charged Particles}

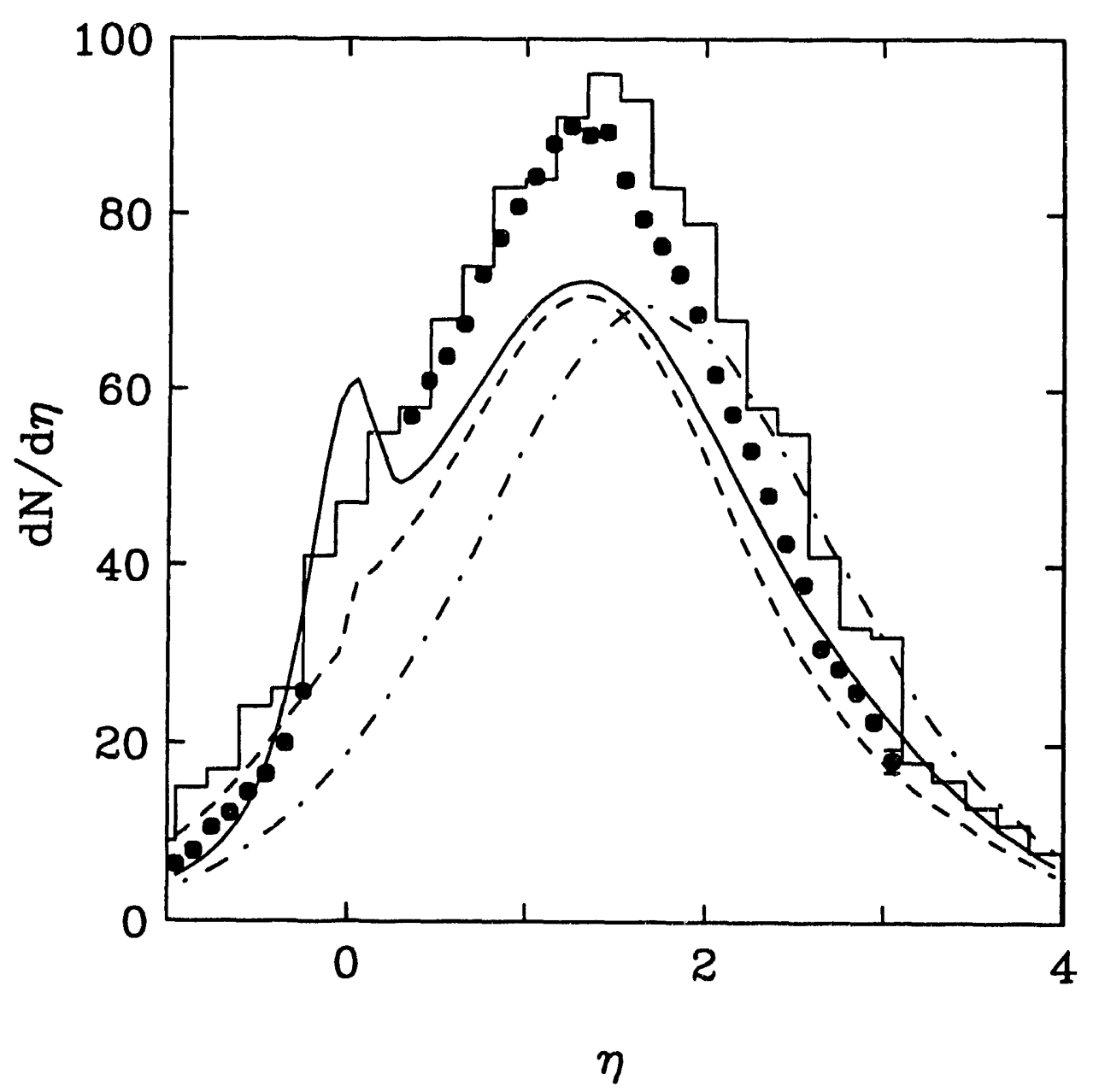

Figure 1.3.4. Fit1 (dashed), fit5 (solid), Attila[Gyu87] (dot-dashed) and RQMD [RQMD91, RQMDpi] (histogram) are compared to preliminary E802 $d N_{\text {chärgeúl }} / d \eta$ data[HIPAGS]. 
for $y>1.5$ and 1.62 for $y<1.5$. From the bottom panels of fig. 1.3.3, it is evident that fit5 is only able to reproduce high rapidity E810[E810] and E814[E814] data and simultaneously account for all of the initial momentum by overpredicting the E802 spectrometer data by $40 \%-70 \%$.

It was pointed out long ago[Stach] that the E802 spectrometer and $d N_{\text {charged }} / d \eta$ data seem to be inconsistent with one another. In fact as can be seen in fig. 1.3.4, our fits significantly underpredict $d N_{\text {charged }} / d \eta$ even though they significantly overpredict the spectrometer data. Only models like RQMD[RQMD91, RQMDpi] which overpredict the spectrometer pions everywhere by at least $70 \%$ are able to accurately reproduce $d N_{\text {charged }} / d \eta$.

\subsubsection{Conclusion}

We conclude that none of the present models which assume complete nuclear stopping and none of the nonequilibrium string models are consistent with the published E802 spectrometer data for central $\mathrm{Si}+\mathrm{Au}$ collisions. If the normalization error of these data does not exceed $30 \%$, then energy-momentum and baryon conservation alone require there to be an unexpected shoulder in the baryon spectrum in the region $2<y<3$ implying a high degree of nuclear transparency. On the other hand, results from E810[E810] and E814[E814] as well as preliminary $d N_{\text {charged }} / d \eta$ data from E802 imply a high degree of nuclear stopping in these reactions. These data seem to be inconsistent with one another, since no model or fit has been found which can reproduce all of the data while preserving 4-momentum conservation. Consequently, until these apparent inconsistencies are resolved, no firm conclusion can be drawn about the amount of nuclear stopping in central $\mathrm{Si}+\mathrm{Au}$ collisions at the AGS. 


\subsection{Recent Developments}

Since the most recent publication of central $\mathrm{Si}+\mathrm{Au} \mathrm{dn} / \mathrm{dy}$ data by the E802 collaboration in 1991[E802], a number of new systematic errors have been found and new low- $p_{\perp}$ measurements have been made which affect these data. The $d n / d y$ yields presented by $\mathrm{E} 802$ at various conferences in the past year and a half have been increasing with time and gradually approaching the values predicted by standard event generators. Although no new $\mathrm{dn} / \mathrm{dy}$ data reflecting these changes have yet been officially sanctioned and published by the E802 collaboration, Chuck Parsons has presented some new data in his May, $1992 \mathrm{PhD}$ thesis[Pars]. In figs. 1.4.1 and 1.4.2, we compare data from Parsons' thesis[Pars] (open circles) with the published 1991 data[E802] (solid dots).

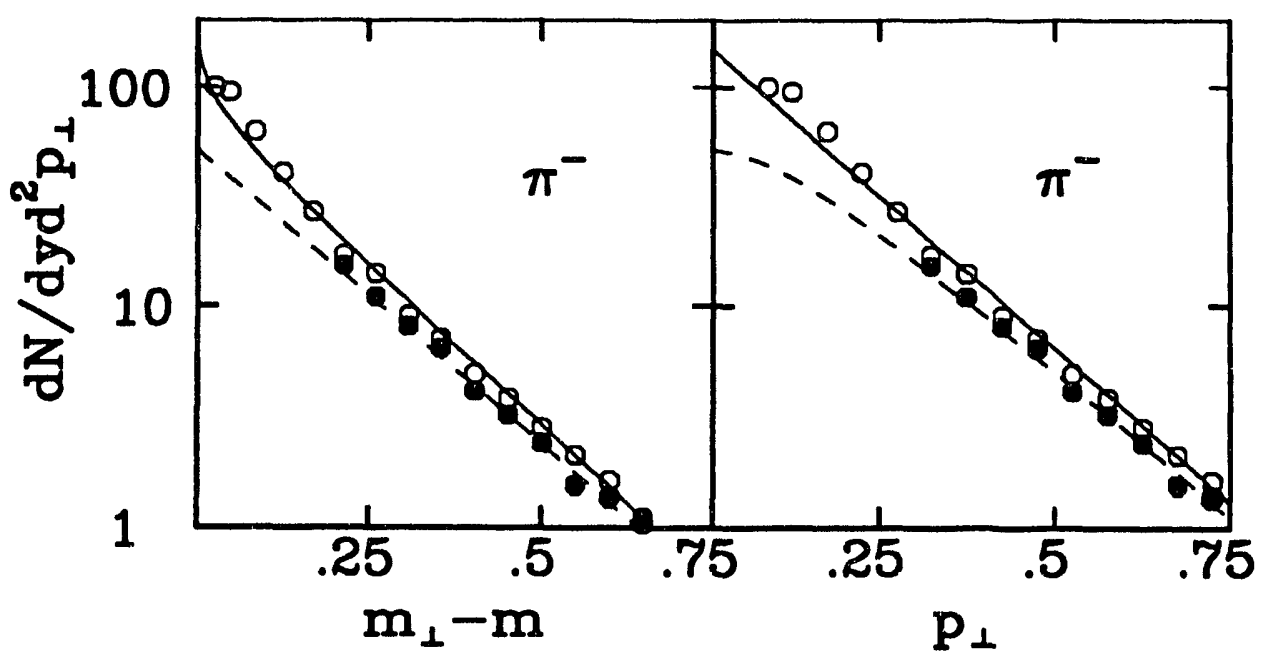

Figure 1.4.1. Solid dots are published invariant cross sections for $\pi^{-}[\mathrm{E} 802]$ at $y=1.3$ in central $\mathrm{Si}+\mathrm{Au}$ collisions, while open circles are recent data presented in the thesis of C. Parsons[Pars]. These data are plotted both as functions of $m_{\perp}-m$ (left) and of $p_{\perp}$ (right). The dashed lines show the exponential $m_{\perp}$ fit used to determine $\mathrm{dn} / \mathrm{dy}$ in [E802], while the solid lines show the exponential $p_{\perp}$ fit used in [Pars].

The most striking feature of the new data is the remarkable increase in midrapidity pion yields. In fig. 1.4.1 we show how the new low- $p_{\perp}$ measurements have motivated fitting the pion invariant cross-sections with $\exp \left(-p_{\perp} / T\right)$ (solid lines), rather than 
with the previously used $\exp \left(-m_{\perp} / T\right)$ (dashed lines). This effect combined with an overall increase in the invariant cross section normalizations is what has lead to the 40-50\% increase in pion $\mathrm{dn} / \mathrm{dy}$ seen in the upper right panel of fig. 1.4.2. For protons, the $30-40 \%$ increase in $\mathrm{dn} / \mathrm{dy}$ seen at high rapidities (upper left panel of fig. 1.4.2) is wholly due to normalization corrections, since the invariant cross sections are still best fit by exponentials in $m_{\perp}$. The slope parameters $(T)$ and Kaon yields have not changed significantly in the new data.

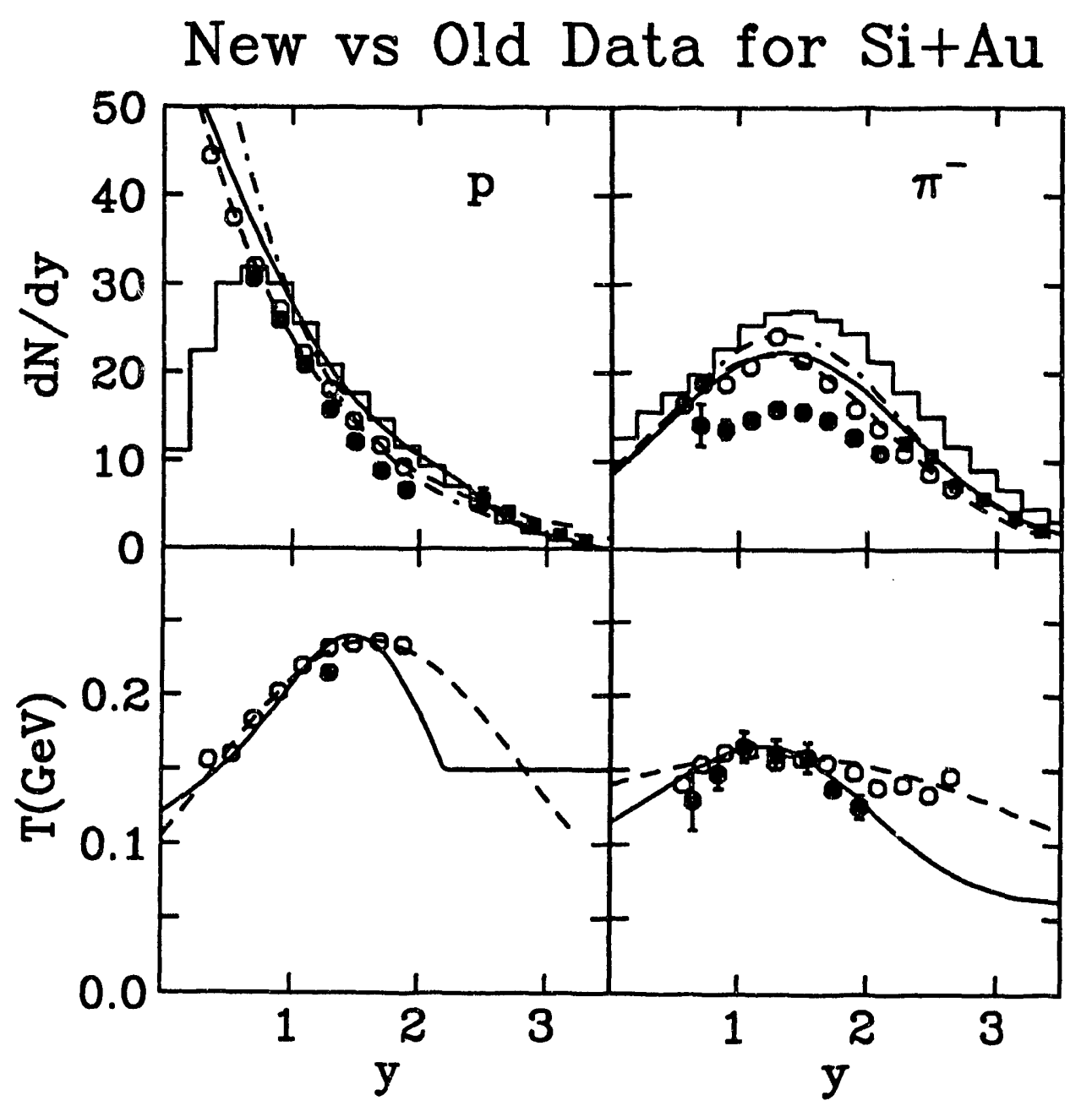

Figure 1.4.2. Solid dots are published $\mathrm{dn} / \mathrm{dy}$ and temperature data for central $\mathrm{Si}+\mathrm{Au}$ collisions[E802], while open circles are from Parsons' thesis[Pars]. Solid squares in the upper left panel are E814 data[E814], while those in the upper right panel are E810 negatives[E810]. These data are compared to Lund calculations[Gyu87] (histogram), as well as to fits by Parsons[Pars] (dashed), Videbaek[Vide] (dot-dashed) and myself (solid). 
The dashed lines in fig. 1.4.2 show fits which Parsons has made to the new data. In his thesis, he performs an analysis in which he claims that 397 out of the initial $409 \mathrm{GeV} / \mathrm{c}$ of longitudinal momentum are accounted for by these fits. However, there are a couple of small problems with his analysis which I will outline here. To find the momentum carried by pions, he used the formula:

$$
P_{\|}=\int d y(d N / d y) \sinh (y) \frac{2 T^{2}+2 T m+m^{2}}{T+m},
$$

where $T(y)$ and $(d N / d y)(y)$ are his fits to the data. Unfortunately, the above formula is only applicable for invariant cross-sections which are fit by exponentials in $m_{\perp}$. The correct formula for cross-sections fit to exponentials in $p_{\perp}$ is the following:

$$
P_{\|}=\int d y(d N / d y) \sinh (y)\left(1 / T^{2}\right) \int d p_{\perp} p_{\perp} \sqrt{p_{\perp}^{2}+m^{2}} \exp \left(-p_{\perp} / T\right)
$$

Using this formula, I find that the pions from his fit carry 117 rather than $126 \mathrm{GeV} / \mathrm{c}$ of longitudinal momentum. The correct treatment features enhanced numbers of low- $p_{\perp}$ pions which do not carry as much longitudinal momentum as their high- $p_{\perp}$ counterparts.

Neutrons are not measured directly by E802, so some ansatz must be made as to their distribution relative to that of the protons. Since the $n / p$ ratios for gold and silicon are 1.5 and 1 respectively, Parsons chose the following ansatz:

$$
\begin{array}{rl}
R_{n / p}=1.5 & y<0.5 \\
R_{n / p}=1 . & y>3.5 \\
R_{n / p}=[(y-0.5)-1.5(y-3.5)] / 3 & 0.5<y<3.5
\end{array}
$$

Using his proton fit along with the above ansatz, Parsons could account for 164 of the original 225 nucleons. He therefore treated the remaining 61 nucleons as spectators located at target rapidity. The $n / p$ ratio of these spectators was never stated, but it can be calculated by integrating the $\mathrm{dn} / \mathrm{dy}$ fits to find the net charge carried by all 
participant hadrons and then comparing this to the initial charge of 93 . The difference in charge must be carried by spectator protons. In this way the spectator $n / p$ ratio can be determined to be 1.28 , which implies an unphysical discontinuity: $n / p=1.28$ for $y=0$ while $n / p=1.5$ for $y>0$. A better ansatz would be the following:

$$
\begin{aligned}
& R_{n / p}=1.423 \quad y<0.5 \\
& R_{n / p}=1 . \quad y>3.5 \\
& R_{n / p}=[(y-0.5)-1.423(y-3.5)] / 3 \quad 0.5<y<3.5
\end{aligned}
$$

which allows the spectators to have a continuous $n / p$ ratio of 1.423 at $y=0$. The neutrons in this scenario carry $126 \mathrm{GeV} / \mathrm{c}$ of $P_{\|}$rather than the $130.4 \mathrm{GeV} / \mathrm{c}$ quoted by Parsons.

The net result of these corrections to Parsons' analysis is that his fits can account for 384 of the initial $409 \mathrm{GeV} / \mathrm{c}$ of $P_{\|}$. Among other ways, the remaining $25 \mathrm{GeV} / \mathrm{c}$ of longitudinal momentum could be accounted for if the overall normalization of all of the new data was low by $6.5 \%$. At any rate, it seems clear that if the high rapidity E810 and E814 proton data are correct, then the absolute normalization of at least some of the published E802 dn/dy data must be low by at least $50 \%$. As a comparison, I show two curves which account for all of the longitudinal momentum as well as Lund model results[Gyu87]. The solid curves in fig. 1.4.2 are from a fit which I presented at Quark Matter 91 (previous section of this thesis), while the dot-dashed curves are derived from a fit made by Flemming Videbaek of the E802 collaboration[Vide]. Videbaek originally fit the published data (solid dots) but then postulated that the true $\mathrm{dn} / \mathrm{dy}$ for pions would be $25 \%$ higher than these data due to undetected low$p_{\perp}$ pions. The fits thus derived could then account for 336 out of $409 \mathrm{GeV} / \mathrm{c}$ of $P_{\|}$. To display the absolute normalizations of $\mathrm{dn} / \mathrm{dy}$ needed to enforce momentum conservation, I have multiplied Videbaek's pion curves by 1.25 and then multiplied both of his curves by an additional 1.22 before plotting them. It is apparent that the increases in normalization of the $\mathrm{dn} / \mathrm{dy}$ data over recent months has gone a long way 
toward solving the problem of momentum conservation, and if they continue at this rate, then it will not be long before we will be justified in concluding that the amount of stopping in these reactions is roughly the same as that in the Lund model.

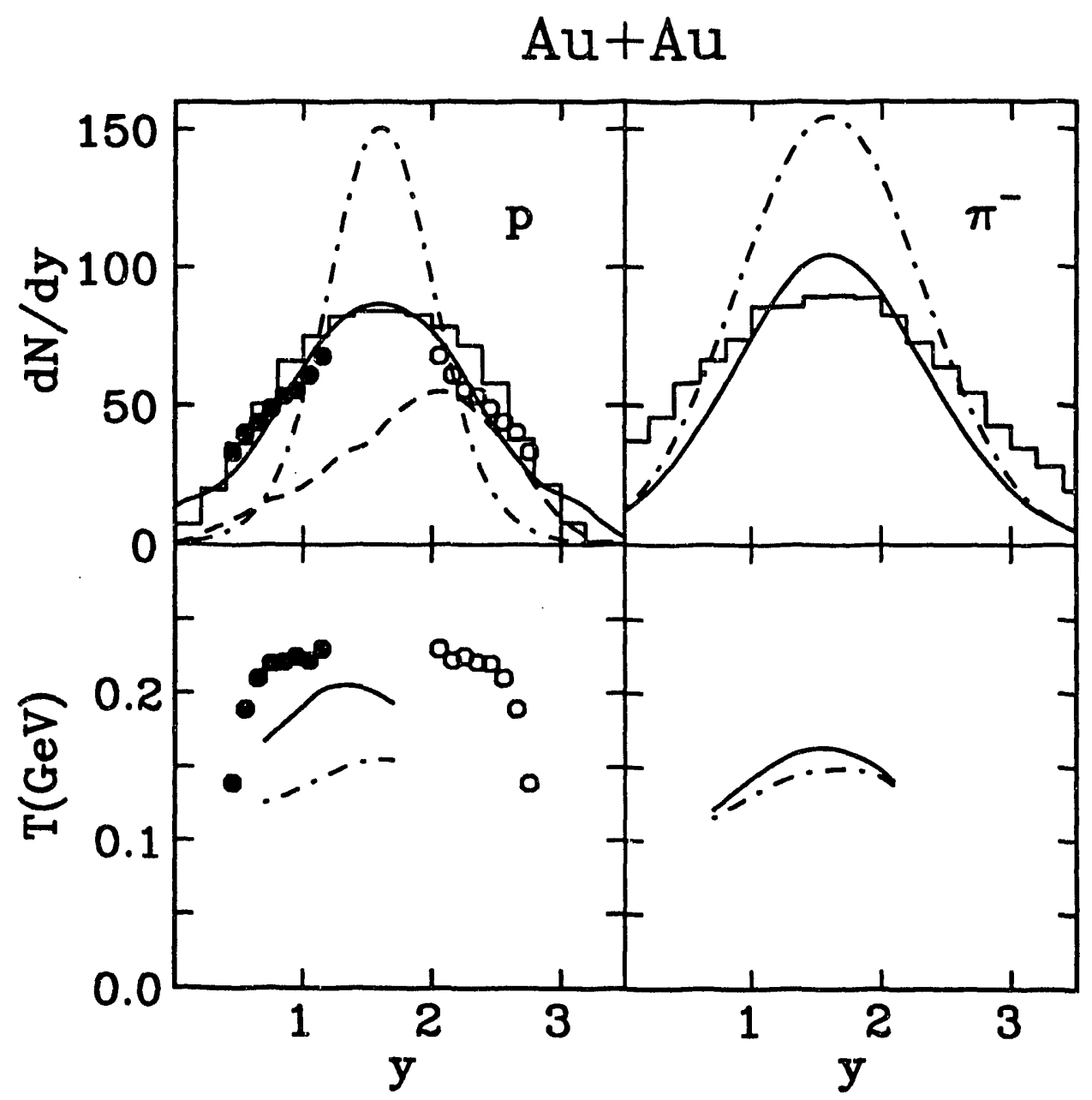

Figure 1.4.3. Solid dots are preliminary data from 11.6 AGeV Au+Au reactions, while open circles are reflections of these data in rapidity due to the symmetry of projectile and target[E866]. These data are compared to Lund model[Gyu87] (histogram), mcml (solid) and firstreak (dot-dashed) calculations. The dashed curve shows the projectile protons only from the Lund calculation.

Recently, some very preliminary $\mathrm{Au}+\mathrm{Au}$ data at $11.6 \mathrm{GeV} / \mathrm{c}$ per nucleon has been presented by the E866 collaboration[E866]. The solid dots in fig. 1.4.3 are the measured data, while the open circles are reflected pseudodata (due to the symmetry of the projectile and target). Both the Lund model[Gyu87] (histogram) and mcml 
(section 2 of this thesis, solid lines) are able to reproduce the gaussian shape of the proton dn/dy remarkably well, even though they are comprised of projectile and target contributions which are still separated by a full unit in rapidity (dashed curve in fig. 1.4.3). Although the fully-stopped firestreak is much too strongly peaked at midrapidity (dot-dashed lines), it will undoubtedly soon be pointed out that a longitudinally expanding fireball (or firestreak) will also be able to reproduce the data. The dilemma, as I stated in my Phys. Rev. C paper (section 2 of this thesis), is that for symmetric collisions, a fully stopped longitudinally expanding fireball cannot be distinguished from two partially stopped fireballs simply on the basis of $\mathrm{dn} / \mathrm{dy}$ data. Certainly, more information is necessary in order to determine the amount of stopping in these reactions.

Another very interesting aspect of the new data is the extremely high apparent temperatures of the protons $(\sim 240 \mathrm{MeV})$. Any statistical model purporting to describe these reactions must necessarily be able to reproduce these temperatures. As can be seen from fig. 1.4.3, a firestreak (or fireball) in which the protons and pions are in equilibrium and freeze out together will feature proton temperatures which are much too low. In order to create high enough temperatures, the protons must freeze out well before the pions as is simulated by $\mathrm{mcml}$, or else there must be an enormous amount of transverse collective flow[Brown]. Without these features, no longitudinally expanding fireball will be able to fit the proton temperatures, even if it can fit the proton $\mathrm{dn} / \mathrm{dy}$ well.

Finally, E866 has measured $K^{+} / \pi^{+}$and $K^{-} / K^{+}$ratios of 0.25 and 0.16 , as compared to 0.19 and 0.28 for $\mathrm{Si}+\mathrm{Au}[\mathrm{E} 866]$. The larger proportion of $K^{+}$'s as compared

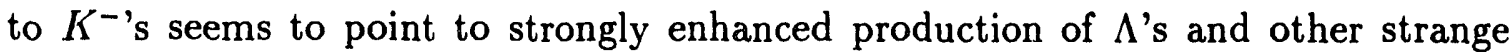
baryons in these reactions. However, from experience with $\mathrm{Si}+\mathrm{Au}$ data, we can see that it is wise not to be too quick in jumping to conclusions based on $K / \pi$ ratios alone. If low- $p_{\perp}$ components and other effects cause a $50 \%$ increase in the pion normalizations without a similar increase for kaons (as seems to have happened for $\mathrm{Si}+\mathrm{Au}$ ), 
then the true $K^{+} / \pi^{+}$ratio may be as low as 0.16 in $\mathrm{Au}+\mathrm{Au}$ collisions. It will be very interesting to see the actual pion and kaon yields which are found so that they can each be directly compared to various models. 


\section{Chapter 2}

\section{Effective Action for SU(N) at Finite Temperature}




\subsection{Introduction}

It has long been predicted that QCD features a phase transition from hadronic matter to a quark-gluon plasma at sufficiently high temperatures or densities[Mor79]. Creating such a plasma is in fact the aim of many of the heavy ion experimental programs at the AGS, SPS and RHIC. Since the quarks and gluons in such a hot plasma would be very energetic, they would also be weakly interacting due to asymptotic freedom. Consequently, extensive work has been done in developing perturbative techniques for finite temperature QCD[Kap79, Bra90]. One of the most interesting results of this perturbation theory is a resummation of infrared divergent diagrams which gives rise to an $A_{0}$ Debye mass of order $g T$ that screens static color electric fields[Kap79]. Unfortunately, no surh resummation has yet been found for the magnetic sector. Consequently, for diagrams above a certain order, infrared divergences become intractable and perturbation theory breaks down[Lin80]. These divergences are a result of loops involving massless $(n=0)$ Matsubara modes, so they do not occur in in QED since the photon only couples to fermions which always have Matsubara frequencies of order $\pi T$.

A constant $A_{0}$ field cannot in general be gauged away at finite temperature the way that it can at zero temperature; consequently quantum effects give rise to an effective potential for the $A_{0}$ field whe: $T>0$ [Wei81]. One way that QCD could generate a magnetic screening mass would be if the $A_{0}$ effective potential were to feature an absolute minimum which was not sirnply a gauge transformation of $A_{0}=0$. The $A_{0}$ field could then possess a nonzero vacuum expectation value (vev), thus behaving like a Higgs field and giving a magnetic mass to the $A_{i}^{a}$ fields through the gauge-gauge coupling terms. Unfortunately, no such minimum exists at the one loop level[Wei81, Gro81]. At the two loop level, on the other hand, the presence of a negative linear term in the effective potential does produce a vev at $A_{0} \sim \mathcal{O}(g T)$, thus giving rise to a magnetic mass of order $g^{2} T$ [Ani84]. This vev and magnetic mass is spurious, however, since the linear term is exactly cancelled by a term arising from the summation of the ring diagrams[Bel91]. Beyond the order of the ring diagrams, 
perturbation theory breaks down due to the magnetic infrared divergences mentioned earlier. There is therefore no way that perturbation theory alone can generate a nontrivial absolute minimum in the $A_{0}$ effective potential. One of the results of this paper is to show in addition that no gauge invariant resummation or non-perturbative technique can give rise to a linear term in the effective potential, since such a term would not be gauge invariant.

In a more general context, it is well known that perturbation theory is limited in its application and by its very nature is not able to shed light on a number of very important unsolved physical problems. For this reason, non-perturbative techniques have been increasingly sought after and explored in recent years. Perhaps the most successful and well-developed of these techniques is the semiclassical method of expanding around classical solutions. In the language of the path integral formalism, the idea behind this method is that by integrating over field configurations which are small fluctuations around nontrivial classical solutions, as well as over ones which are close to the perturbative vacuum, one can better approximate the full functional integral, which should in principle be performed over all possible field configurations. In field theory for example, integrating around instanton solutions allows one to gain insight into quantum tunneling processes which can never be described by any finite order of perturbation theory[Raj82, Col77, tHo76].

Similarly, for finite temperature QCD, it has been pointed out that integrating around a plasma of magnetic monopoles could possibly provide magnetic screening as $T \rightarrow \infty$ [Gro81, Dah85, Polo91]. Is there any evidence for the presence of such monopoles? At zero temperature, Mandelstam showed that if the ground state of QCD is a coherent superpositicn of monopoles, then confinement could be understood as the dual analog of superconductivity[Man76]. In other words, just as a condensate of electrically charged Cooper pairs will adjust to confine magnetic fields inside a superconductor, a condensate of color magnetic charges would adjust to confine color electric fields in the QCD vacuum. It has never been proven that such a condensate actually forms the ground state of QCD, however Savvidy has shown that a constant color magnetic field $H$ has negative energy compared to the perturbative vacuum at 
$T=0[$ Sav77]. Although Savvidy's configuration violates Lorentz invariance, his result suggests that the ground state of QCD does have some nontrivial magnetic structure. Based on this idea, Oleson has advocated a picture in which random distributions of magnetic vortices form a Lorentz invariant ground state featuring $\langle H\rangle=0$ but $<H^{2}>\neq 0$ [Ole81]. This picture is not contradictory with one involving a monopole condensate since magnetic vorices of finite length must begin and end at monopoles, and both pictures feature strongly enhanced low frequency fluctuations[Man76]. At high temperatures, even though the Savvidy effect disappears[Mul85], the presence of low frequency (infrared) magnetic instabilities could be indicating the presence of monopoles or other magnetically charged objects. In this paper, we consider only high temperature monopoles and dyons and do not specifically address condensate formation or other issues relating to confinement at $T=0$.

In $\mathrm{SU}(\mathrm{N})$ at zero temperature the $A_{0}^{a}$ field can always be gauged away, so if there are monopole solutions, one must be able to create them from the $A_{i}^{a}$ fields alone. Infinite energy monopole solutions and finite energy monopole configurations which are not solutions have been found[Wu68, Col81], but no finite energy monopole solutions are known for $T=0$. In order to find a solution which sufficiently smoothes out the $1 / r$ singularities in the $A_{i}$ fields at the origin, one usually introduces a scalar field in the adjoint representation, as is done for the 't Hooft-Polyakov[tHo74, Poly75] or Prasad-Sommerfield[Pra75] monopoles. At finite temperature, however, the $A_{0}^{a}$ field cannot in general be gauged away, and it is therefore able to play the role of the Higgs field in a monopole configuration. Making this substitution, PrasadSommerfield monopoles become dyon solutions in pure gauge theories, possessing electric as well as magnetic charge. Although the dual charge of dyons makes them necessarily more complicated than monopoles, they are at present the only available magnetically charged classical solutions with finite energy at the tree level, so they are a logical object of study. In addition to knowing the classical mass of these dyons, it is obviously important to know how dense of a gas or plasma they might form at any given temperature.

Finding the density of a soliton plasma can be a highly nontrivial task. In order to 
derive an expression for the density, I will present a brief outline of the semiclassical method for a field theory at finite temperature. The first step is to find a nontrivial field configuration with energy $E_{1}<\infty$ which is a local minimum of the classical action $^{1}$. Since the solution has finite energy, it must be localized, approaching the perturbative vacuum solution (or one of them if there is more than one) as $r \rightarrow \infty$. For the sake of simplicity, we will assume that the solution is a time independent soliton. It is plausible that a configuration with two solitons which are separated by a distance much larger than their size would be a close approximation to another solution. One therefore proceeds by either proving or assuming that configurations with $\mathrm{N}$ well-separated identical solitons are also local minima of the action[Raj82, Poly77, War81, Cor81]. Often it is shown or assumed in addition that the solitons are weakly interacting. If this is the case, then the relative positions of solitons in an $\mathrm{N}$ soliton configuration are arbitrary and must be integrated over as well, giving a factor of volume $V$ for each soliton. Putting together these ideas, one can write down a rough approximation to the partition function of a plasma of these solitons[Poly77]:

$$
Z=1+\gamma T^{3} V \exp \left(-E_{1} / T\right)+\frac{1}{2 !}\left[\gamma T^{3} V \exp \left(-E_{1} / T\right)\right]^{2}+\ldots
$$

where the first term corresponds to no solitons, the second to one soliton, the third to two, etc. Since the position of each identical soliton is being integrated over, a symmetry factor of $\frac{1}{N !}$ must be included for $N$ soliton configurations. In addition, there is a dimensionless "prefactor" $\gamma$ included for each soliton which can in general be some complicated function of the coupling constant $g$.

The density of a plasma of solitons is determined by noting that for $Z=\sum x^{n} / n$ !, the average $\mathrm{n}$ is given by $\langle n\rangle=x$. Thus, the density is simply:

$$
<n>/ V \sim \gamma T^{3} \exp \left(-E_{1} / T\right)
$$

To determine $\gamma$ in the one loop approximation, one must calculate functional determinants around a soliton background. This is a very formidable task since no general

\footnotetext{
'it is not enough to find a classical solution vinich maximizes the action in some functionai direction, because integrating over all small fluctuations around such a configuration would give an infinite result. This is the problem of negative eigenmodes which we address later in the paper.
} 
method is known for calculating these determinants exactly. For this reason, the value of $\gamma$ is often simply estimated by heuristic arguments.

The main thrust of this paper is to develop new approximation techniques for determining prefactors $\gamma$ around a large class of background configurations at finite temperature. As examples of their utility, these techniques are applied to dyon and monopole configurations in pure gauge $\mathrm{SU}(2)$. It is found that if plasmas of such configurations do exist, then either they are unstable, infinitely massive, or else their density is so high that they are strongly overlapping. For these types of configurations, semiclassical techniques are therefore not applicable. However, it is not ruled out that other soliton solutions may be found in the future which are not plagued by the above problems. In that event, the density of plasmas of those solitons could then be found by using the techniques developed here. For example, magnetically charged meron-antimeron solutions are known to exist at finite temperature[Jon81], though no explicit solutions are available. Alternatively for pure gauge theories, Coleman has found topologically stable monopole solutions which have a singularity at the origin[Col81]. It is possible that singularity-free monopole configurations could be found which would approach the above solutions as $r \rightarrow \infty$ and would also minimize the one loop effective action.

I begin this paper by presenting the basic notation and formulas for finding th regularized one loop effective action for a pure gauge non-Abelian theory. Next, 1 generalize the methods of ref. [Dya84] to finite temperature and derive a covariant derivative expansion for the effective action. The increasing dimension of successive terms of this expansion is balanced by an infrared cutoff mass which is self consistently determined so as to optimize the expansion. This infrared scale is shown to uniquely determine the semiclassical prefactor $\gamma$. Comparison of the results of the expansion to the known effective potential for a constant $A_{0}^{a}$ field in $\mathrm{SU}(2)$ suggests that the lowest order form of this expansion should be reliable for slowly varying configurations in which $\left|A_{0}^{a}\right|<\mathcal{O}(T / g)$ when $g \rightarrow 0(T \rightarrow \infty)$. After showing that dyons meeting the above qualifications must necessarily be overlapping, I extend the covariant derivative expansion by performing a resummation in order to find an expression which is valid 
for static background configurations with $\left|A_{0}^{a}\right|=\mathcal{O}(T / g)$. Since the effective potential of the $A_{0}^{a}$ field can have periodic minima at $4 n \pi T / N g$ for pure gauge $\mathrm{SU}(\mathrm{N}$ ) (see Appendix B), I also examine dyon solutions and monopole configurations in which the magnitude of the $A_{0}^{a}$ field approaches one of these minima as $r \rightarrow \infty$. I show that these monopoles are unstable and that depending on the temperature, the corresponding dyons are either infinitely massive or else overlapping. I complete the discussion of monopoles and dyons by showing that introducing fermions into the theory does not improve the situation.

\subsection{Preliminaries}

We consider a pure gauge, Euclidean, non-Abelian theory with the Lagrangian:

$$
\mathcal{L}=-\frac{1}{4}\left(F_{\mu \nu}^{a}\right)^{2}
$$

where

$$
F_{\mu \nu}^{a}=\partial_{\mu} A_{\nu}^{a}-D_{\nu}^{a b} A_{\mu}^{b}
$$

and

$$
D_{\mu}^{a b}=\partial_{\mu} \delta^{a b}-g f^{a b c} A_{\mu}^{c} .
$$

Since we are interested in finite temperature, the fields have periodic temporal boundary conditions $A_{\mu}^{a}(\tau)=A_{\mu}^{a}(\tau+\beta)$, where $\beta=1 / T[\operatorname{Kap} 79]$. The equations of motion for this Lagrangian are

$$
D_{\mu}^{a b} F_{\mu \nu}^{b}=0
$$

Let $\bar{A}_{\mu}^{a}$ be solutions to the above equations which transform as normal Yang-Mills gauge fields, and let $B_{\mu}^{a}$ be quantum fluctuations around those solutions which transform in the adjoint representation. To consider one loop effects, we make the substitution[deW67] $\left(A_{\mu}^{a}=\bar{A}_{\mu}^{a}+B_{\mu}^{a}\right)$ in the Lagrangian and expand the action up to terms quadratic in $B_{\mu}^{a}$ :

$$
S(\bar{A}+B)=\bar{S}-\frac{1}{2} \int d^{4} x B_{\mu}^{a} W_{\mu \nu}^{a b} B_{\nu}^{b}
$$


where $\bar{S}=S(\bar{A})$. Note that there are no terms linear in $B$ since $\bar{A}$ is a classical solution and hence a saddle point. We choose to work in the background gauge,

$$
\bar{D}_{\mu}^{a b} B_{\mu}^{b}=0,
$$

where $\bar{D}_{\mu}^{a b}=\partial_{\mu} \delta^{a b}-g f^{a b c} \bar{A}_{\mu}^{c}$, since it is manifestly covariant and because Pauli-Villars regularization takes a particularly simple form in this gauge[Sla80]. By adding a gauge fixing term of $\frac{1}{2}\left(\bar{D}_{\mu} B_{\mu}\right)^{2}$ to the Lagrangian, we get:

$$
W_{\mu \nu}^{a b}=-\left(\bar{D}^{2}\right)^{a b} \delta_{\mu \nu}+2 g f^{a b c} \bar{F}_{\mu \nu}^{c},
$$

where $\bar{F}_{\mu \nu}^{a}=\partial_{\mu} \bar{A}_{\nu}^{a}-\bar{D}_{\nu}^{a b} \bar{A}_{\mu}^{b}$.

The functional integral needed to calculate the one loop effective potential is given by:

$$
Z[\bar{A}]=e^{\bar{S}} \int\left[\mathcal{D} B_{\mu}^{a} \bar{\xi} \xi\right] \exp \left\{-\int d^{4} x\left[\frac{1}{2} B_{\mu}^{a} W_{\mu \nu}^{a b} B_{\nu}^{b}-\bar{\xi}^{a}\left(-\bar{D}^{2}\right)^{a b} \xi^{b}\right]\right\}
$$

Pauli-Villars regularization can be performed by introducing auxiliary fields $B^{\prime}$ and $\xi^{\prime}$ which transform like $B_{\mu}^{a}$ and $\xi^{a}$, but have mass $\Lambda$ which will later be allowed to become infinite. Because all of the field fluctuations are in the adjoint representation, the mass terms $\Lambda^{2} B^{\prime 2}$ and $\bar{\xi}^{\prime} \Lambda^{2} \xi^{\prime}$ are gauge invariant. Application of this procedure produces the following regulated partition function[Sla80]:

$$
\left.Z[\bar{A}]\right|_{\mathrm{reg}}=Z[\bar{A}] / Z^{\prime}\left[\bar{A}, \Lambda^{2}\right]
$$

where $Z^{\prime}$ has the same form as eqn. (2.2.8), except that mass terms are included. Note that for convenience we have used the same Pauli-Villars mass $\Lambda$ for both the $B^{\prime}$ and $\xi^{\prime}$ fields.

\subsubsection{Zero Modes}

We assume that the classical solutions depend on $p$ parameters $\gamma_{i}$ but that the total gauge-fixed action is independent of these parameters. There are then $p$ remaining zero modes of the Lagrangian given by $\partial \bar{A}_{\mu}^{a} / \partial \gamma_{i}$ where $i$ runs from 0 to $p$. Actually, 
any gauge transformation of one of these modes will also be a zero mode, so a more general expression for these zero modes is[Poly77]:

$$
\sqrt{N_{i}} \chi_{\mu}^{a(i)}=\partial \bar{A}_{\mu}^{a} / \partial \gamma_{i}+D_{\mu}^{a b} \theta_{i}^{b}
$$

where the second term is pure gauge and $\theta_{i}^{b}$ are gauge functions. By fixing the gauge, we have already removed all of the modes which do sut satisfy (2.2.6), so in order to determine the remaining zero modes, we need to find specific functions $\theta_{i}^{b}$ such that $\bar{D}_{\mu}^{a b} \chi_{\mu}^{b(i)}=0$. For the cases that we are studying, these zero modes can be made to be orthonormal, so we will demand:

$$
\int d^{4} x \chi_{\mu}^{a(i)} \chi_{\mu}^{a(j)}=\delta^{i j}
$$

As a concrete example, consider a soliton solution which is centered around some point in space denoted by the vector $\vec{R}$. The solution then has the form $\bar{A}_{\mu}^{a}=\bar{A}_{\mu}^{a}(\tau, \vec{x}-\vec{R})$. Since the Lagrangian has no preferred points, a change in $\vec{R}$ will not change the action and therefore represents a zero mode. In this case $\gamma_{i}=R_{i}$, and due to the functional form of the solution $\partial \bar{A}_{\mu}^{a} / \partial R_{i}=-\partial_{i} \bar{A}_{\mu}^{a}$. We then choose $\theta_{i}^{b}=\bar{A}_{i}^{b}$ so that

$$
\sqrt{N_{i}} \chi_{\mu}^{a(i)}=-\bar{F}_{\mu i}^{a}
$$

and the background gauge requirement (2.2.6) is trivially satisfied by the equations of motion (2.2.4). The normalization for this mode is then given by:

$$
N_{i}=\int d^{4} x\left(\bar{F}_{\mu i}^{a}\right)^{2},
$$

where $i$ is a label which is not summed over. If the soliton is a self-dual solution, then[Act79]

$$
E_{i}^{a} \equiv F_{0 i}^{a}=-\frac{1}{2} \epsilon_{i j k} F_{j k}^{a} \equiv B_{i}^{a}
$$

If, in addition, the soliton is spherically symmetric, then the normalization takes the remarkably simple form of $N_{i}=-\bar{S}$.

There are infinities due to the functional integration over non-gauge zero modes which call be isolated by the collective coordinate method[Raj82, Poly77]. First, we expand an arbitrary field configuration as follows:

$$
A(x)=\bar{A}\left(x, \gamma_{i}\right)+\sum_{n} \xi_{n} b_{n}(x)
$$


where $b_{n}$ are orthonormal eigenfunctions of $W$ with positive eigenvalues, and we have explicitly included the $\gamma_{i}$ dependence of $\bar{A}$ in order to allow for zero mode fluctuations. To perform the functional integration, we must find the Jacobian associated with expressing the metric in terms of the eigenfunctions. For finite matrices, the Jacobian for a transformation from a vector $X$ in one basis to a vector $Y$ in another basis is found by calculating $\operatorname{det} J$, where $\delta X=J \delta Y$. If both bases under consideration are orthogonal, then $J$ can always be diagonalized by a unitary transformation $J^{\prime}=$ $U J U^{-1}$ so that the determinant is given by $\operatorname{det} J=\prod_{k} J_{k k}^{\prime}$. Calculating the length element then defines the determinant by isolating the diagonal elements of $J^{\prime}$ :

$$
(\delta \ell)^{2}=(\delta X)^{2}=\left(J^{\prime} U \delta Y\right)^{2}=\sum_{k} J_{k k}^{\prime 2}(\delta Y)^{2}
$$

Generalizing this technique to field theory and applying it to our problem, we have:

$$
(\delta \ell)^{2}=\int d^{4} x(\delta A(x))^{2}=\sum_{i=1}^{p} N_{i}\left(\delta \gamma_{i}\right)^{2}+\sum_{n}\left(\delta \xi_{n}\right)^{2},
$$

so that after Gaussian integrations[Poly77],

$$
Z[\bar{A}]=\left(\prod_{i=1}^{p} \sqrt{N_{i}} \int d \gamma_{i}\right) e^{\bar{s}} \operatorname{det}\left(-\bar{D}^{2}\right)\left[\operatorname{det}^{\prime}(W / 2 \pi)\right]^{-1 / 2}
$$

where $\operatorname{det}^{\prime}(W / 2 \pi)$ means to take the determinant with respect to the nonzero modes of $W / 2 \pi$ only.

Since we are using Pauli-Villars regulators, we will also encounter the operators $W+\Lambda^{2}$, which do not have any zero modes. When taking determinants of these, however, it is still convenient to split the results into two factors:

$$
\operatorname{det}\left[\left(W+\Lambda^{2}\right) / 2 \pi\right]^{-1 / 2}=\left(\frac{2 \pi}{\Lambda^{2}}\right)^{p / 2} \operatorname{det}^{\prime}\left[\left(W+\Lambda^{2}\right) / 2 \pi\right]^{-1 / 2} .
$$

The full regulated expression, therefore becomes:

$$
\left.Z[\bar{A}]\right|_{\mathrm{reg}}=\left.\left.\left(\prod_{i=1}^{p} \sqrt{N_{i}} \int d \gamma_{i}\right)\left(\frac{\Lambda^{2}}{2 \pi}\right)^{p / 2} e^{S(\bar{A})} \operatorname{det}\left(-\bar{D}^{2}\right)\right|_{\mathrm{reg}}\left[\operatorname{det}{ }^{\prime}(W)\right]\right|_{\text {reg }} ^{-1 / 2},
$$

where we use the following notation for some operator $\mathrm{K}$ :

$$
\left.\operatorname{det}(K)\right|_{\mathrm{reg}}=\frac{\operatorname{det}(K)}{\operatorname{det}\left(\boldsymbol{\Lambda}^{\prime}+\Lambda^{2}\right)}
$$


Note that we have left out the factors of $2 \pi$ in the regulated determinants of eqn. (2.2.20), since this expression only involves ratios of determinants, and multiplicative constants drop out. For the remainder of this paper we will drop the bars on $\bar{A}, \bar{D}$ and $\bar{F}$ except where they are needed for clarity, keeping in mind that we are always referring to functions of the background field and not of the full field with quantum fluctuations included.

\subsection{Covariant Derivative Expansion}

Now comes the difficult problem of evaluating the functional determinants. For a few select cases, the determinants can be evaluated exactly, but in order to find a general expression, some approximation procedure must be used. The most common method is to make a covariant derivative expansion. There have been many papers written suggesting a variety of ways to make such an expansion at zero termprature[Dya84, Che87, Chan86, Gai89], but the literature on finite temperature expansions is much more limited[Min86]. Each of the zero temperature methods that deals with a massless theory is forced to introduce some form of infrared cutoff mass in order to balance the dimension of new derivative terms. In most schemes, this cutoff mass remains unspecified with the argument that in a complete calculation of an observable it will drop out anyway. Alternatively, D'yakonov et al.[Dya84] proposed a scheme in which the infrared cutoff is actually chosen in such a way that it optimizes the accuracy of any desired order of derivative expansion. To check their method, they calculated the one loop quantum correction to the action of the SU(2) instanton and obtained a result which was within $3 \%$ of the exact value calculated by 't Hooft[tHo76]. It is this method that we have chosen to extend to finite temperatures.

In order to determine the free energy $\Omega$ of some nontrivial background configuration $A$, we need to calculate the ratio of the partition function of that configuration to the trivial $A=0$ configuration:

$$
\exp (-\Omega / T)=\left.\frac{Z(A)}{Z(0)}\right|_{\mathrm{reg}} .
$$


From eqn. (2.2.20), we can see that the calculation will entail finding ratios of determinants of various operators. These ratios can be evaluated by using the following expression for the difference of two logarithms:

$$
\left.\frac{\operatorname{det} K}{\operatorname{det} K_{0}}\right|_{\text {reg }}=\exp \left\{-\int_{0}^{\infty} \frac{d t}{t} R(t) \operatorname{Tr}\left(e^{-t K}-e^{-t K_{0}}\right)\right\}
$$

where $\operatorname{Tr}$ is a functional trace over all indices and coordinates and

$$
R(t)=1-e^{-t \Lambda^{2}} .
$$

Note that $t$ is formally of dimension $M^{-2}$. As long as both of the operators that we are interested in $\left(-D^{2}, W\right)$ are positive definite, they will have continuous spectra of eigenvalues beginning with zero, as do their vacuum operator counterparts $\left(-\partial^{2},-\partial^{2}\right)$. One expects, therefore, that for sufficiently smooth and rapidly falling background fields, the integrand of (2.3.2) will be a rapidly decaying function of $t$ [Dya84]. This suggests the possibility of an approximation whereby the infinite upper limit of the $t$ integration is replaced by an infrared cutoff $\delta$. In addition to this approximation, we will make an expansion of the exponential operators in powers of covariant derivatives. After integrating with respect to $t$, the optimum $\delta$ for any given number of terms in this expansion can be determined by finding the extremum in the resulting expression.

The functional trace in eqn. (2.3.2) can be taken relative to any complete set of states, so we are free to use plane waves $\exp \left(i p_{\alpha} x_{\alpha}\right)$. These have the effect of shifting the derivatives:

$$
\operatorname{Tr} e^{-t K}=\operatorname{tr} \int d^{4} x T \sum_{n} \int \frac{d^{3} p}{(2 \pi)^{3}} \exp \left[-K\left(\partial_{\alpha} \rightarrow \partial_{\alpha}+i p_{\alpha}\right) t\right] 1,
$$

where $\operatorname{tr}$ is a simple trace over spacetime and color indices. Due to the periodic temporal boundary conditions, we have replaced the normal zero temperature $p_{0}$ integral for a sum over the modes $p_{0}=2 n \pi T$. Also the $x_{0}$ integral in $d^{4} x$ is from 0 to $\beta=1 / T$. A 1 has been included at the end of the equation to emphasize the fact that the shifted $\exp (-K t)$ operates on unity; so that, for example, any term in the expansion of the exponent with a $\partial_{\alpha}$ all the way to the right will vanish. 


\subsubsection{Ghosts}

We now present the covariant derivative expansion of the ghost determinant. According to eqn. (2.3.4), we have

$$
I^{g h}=\operatorname{Tr} \exp \left(D^{2} t\right)=\operatorname{tr} \int d^{4} x T \sum_{n} \int \frac{d^{3} p}{(2 \pi)^{3}} e^{-p^{2} t} \exp \left[\left(D^{2}+2 i p_{\alpha} D_{\alpha}\right) t\right] 1
$$

The expansion amounts to expressing

$$
I^{g h}=\sum_{n} I_{n}^{g h}
$$

where $I_{n}^{g h}$ is comprised of terms involving $n$ covariant derivatives. $I_{0}^{g h}$ is simply given by the zeroth order term in the $t$ expansion of eqn. (2.3.5), but is exactly cancelled in our calculation by the vacuum contribution seen in eqn. (2.3.2). Moreover, any term in the expansion with an odd number of $D_{\alpha}$ 's will vanish upon $p$ integration.

Thus the first nonzero term in the covariant derivative expansion is given by:

$$
\begin{aligned}
I_{2}^{g h} & =\operatorname{tr} \int d^{4} x T \sum_{n} \int \frac{d^{3} p}{(2 \pi)^{3}} e^{-p^{2} t}\left[D^{2} t+\frac{(2 i)^{2}}{2 !} p_{\alpha} p_{\beta} D_{\alpha} D_{\beta} t^{2}\right] \mathbf{1} \\
& =\frac{t}{(4 \pi t)^{3 / 2}}\left(\operatorname{tr} \int d^{4} x D_{0}^{2}\right) T \sum_{n}\left(1-2 p_{0}^{2} t\right) \exp \left(-p_{0}^{2} t\right),
\end{aligned}
$$

where we have performed the momentum integral by using equations (A.1) and (A.2) in Appendix A. We would like to separate the $T=0$ and $T \neq 0$ parts of the above expression. This can be done by using equations (A.4) and (A.5) which have been derived from the Poisson summation formula (A.7):

$$
I_{2}^{g h}=\frac{H_{1}}{8 \pi^{2} t} \sum_{n} \frac{n^{2}}{4 T^{2} t} \exp \left(-\frac{n^{2}}{4 T^{2} t}\right)
$$

where

$$
H_{1}=\operatorname{tr} \int d^{4} x D_{0}^{2}=-g^{2} N \int d^{4} x A_{0}^{2} .
$$

Each term in eqn. (2.3.7) vanishes in the $T \rightarrow 0$ limit. This is reassuring since $H_{1}$ can be gauged away in the $T=0$ limit. 
Using similar techniques, the next term in the expansion is given by:

$$
I_{4}^{g h}=\frac{1}{48 \pi^{2}} \sum_{n} \exp \left(-\frac{n^{2}}{4 T^{2} t}\right)\left[\frac{1}{4} F_{2}+\left(\frac{n^{2}}{4 T^{2} t}\right)\left(G_{2}-D_{2}\right)+2\left(\frac{n^{2}}{4 T^{2} t}\right)^{2} H_{2}\right],
$$

with the functionals $F_{2}, D_{2}, G_{n}$ and $H_{n}$ defined in Appendix A. Here the only term surviving when $T \rightarrow 0$ is the $F_{2}$ term $^{2}$ with $n=0$, in agreement with the result of d'Yakonov et al. [Dya84]. This expansion can of course be continued, but for our purposes we will only need the first two terms.

To find the determinant, we must integrate over $t$ as in eqn. (2.3.2). In all of our expressions, the zero temperature $(n=0)$ terms are the only ones with ultraviolet divergences. For the rest however, we can immediately let $\Lambda \rightarrow \infty$ so that $R(t)=1$ and perform the remaining elementary integrals by using the variable $u=1 / t$. For the case of $I_{2}^{g h}$ we get:

$$
\begin{aligned}
\int_{0}^{\delta} \frac{d t}{t} I_{2}^{g h} & =\frac{H_{1}}{4 \pi^{2}} \sum_{n=1}^{\infty} \exp \left(-\frac{n^{2}}{4 T^{2} \delta}\right)\left[1 / \delta+\frac{4 T^{2}}{n^{2}}\right] \\
& \simeq \frac{1}{6} T^{2} H_{1}-\frac{T}{4 \sqrt{\pi^{3} \delta}} H_{1}
\end{aligned}
$$

where the second equality is found after using the approximate expressions in Appendix A which become exact as $4 T^{2} \delta \rightarrow \infty$. In this paper, we will only consider infrared cutoffs $T^{2} \delta \sim \mathcal{O}\left(1 / g^{\alpha}\right)$ with $\alpha \geq 0$. For $\alpha>0$, the approximations used are obviously very good at high temperatures, but surprisingly enough, even when $4 T^{2} \delta=1$, they are accurate to within a few percent. On the other hand, these approximations are not valid for $T=0$, and consequently many of the following equations will not reduce correctly to their zero temperature counterparts in the limit as $T \rightarrow 0$. After using eqns. (A.16-A.19) to perform the $t$ integration and high temperature approximations on $I_{4}^{g h}$, we arrive at the following expression for the regulated ghost determinant:

\footnotetext{
${ }^{2}$ Note that after using the Poisson summation formula, the sum over $n$ is no longer a sum over Matsubara frequencies; in fact, $n=0$ terms correspond to $T=0$, while the $n \neq 0$ terms provide the temperature corrections
} 


$$
\begin{aligned}
\left.\ln \left[\frac{\operatorname{det}\left(-D^{2}\right)}{\operatorname{det}\left(-\partial^{2}\right)}\right]\right|_{\text {reg }} & \simeq-\left(\frac{T^{2}}{6}-\frac{T}{4 \sqrt{\pi^{3} \delta}}\right) H_{1}-\frac{T \sqrt{\delta}}{48 \sqrt{\pi^{3}}}\left[F_{2}+2\left(G_{2}-D_{2}\right)+6 H_{2}\right] \\
& -\frac{1}{48 \pi^{2}}\left\{\frac{1}{4}\left[\gamma_{E}-3.1+\ln \left(\frac{\Lambda^{2}}{4 T^{2}}\right)\right] F_{2}-G_{2}+D_{2}-2 H_{2}\right\}
\end{aligned}
$$

\subsubsection{Gauge Fields}

For the gauge fields, we must only take the trace over the nonzero modes of $\exp (-W t)$. If, however, we take the trace over all eigenfunctions of $W, p$ of them will just give us a 1 . This contribution can be subtracted out by hand, so that we get:

$$
\left.\frac{\operatorname{det}^{\prime} W}{\operatorname{det} W_{0}}\right|_{\text {reg }}=\exp \left\{-\int_{0}^{\infty} \frac{d t}{t} R(t)\left[\operatorname{Tr}\left(e^{-t W}-e^{-t W_{0}}\right)-p\right]\right\} 1
$$

Since the trace is now over all modes, we can just take it with respect to the functions $b_{\mu}^{a} \exp \left(i p_{\alpha} x_{\alpha}\right)$, where $b_{\mu}^{a} b_{\nu}^{b}=\delta_{\mu \nu}^{a b}$. The calculations for gauge fields are similar to the ones for ghosts and one finds:

$$
\begin{aligned}
\left.\ln \left[\frac{\operatorname{det}(W)}{\operatorname{det}\left(-\partial^{2}\right)}\right]\right|_{\text {reg }} & =\left.4 \ln \left[\frac{\operatorname{det}\left(-D^{2}\right)}{\operatorname{det}\left(-\partial^{2}\right)}\right]\right|_{\text {reg }}+p\left[\gamma_{E}+\ln \left(\Lambda^{2} \delta\right)\right] \\
& +\frac{1}{8 \pi^{2}}\left[\gamma_{E}-3.1+\ln \left(\frac{\Lambda^{2}}{4 T^{2}}\right)+4 T \sqrt{\pi \delta}\right] F_{2}
\end{aligned}
$$

Note that if $F_{2}=p=0$ (as for a constant field), then $W_{\mu \nu}=-D^{2} \delta_{\mu \nu}$ and the log of the gauge determinant is simply 4 times that of the ghosts, since the former involves a trace over spacetime indices.

From eqn. (2.2.20), we can see that the quantity that we will be interested in will be

$$
\left.\ln \left[\frac{\operatorname{det}\left(-D^{2}\right)}{\operatorname{det}\left(-\partial^{2}\right)}\right]\right|_{\text {reg }}-\left.\frac{1}{2} \ln \left[\frac{\operatorname{det}(W)}{\operatorname{det}_{(}\left(-\partial^{2}\right)}\right]\right|_{\text {reg }} \text {. }
$$

Using the expressions in (2.3.11) and (2.3.13), we can optimize the derivative expansion by differentiating (2.3.14) with respect to $\delta$ and finding an extremum. The resulting $\delta$ must obey the equation:

$$
12 H_{1}+\left[-11 F_{2}+2\left(G_{2}-D_{2}\right)+6 H_{2}\right] \delta-48 p \sqrt{\pi^{3} \delta} / T=0 .
$$


Plugging in this $\delta$, we get:

$$
\frac{Z}{Z_{0}}=\left(\prod_{i=1}^{p} \sqrt{N_{i}} \int d \gamma_{i}\right)\left(\frac{1}{2 \pi \delta}\right)^{p / 2} \exp \left(S_{\text {eff }}\right),
$$

where

$$
\begin{aligned}
S_{\text {eff }} & =\frac{1}{4 N g^{2}} F_{2}-\frac{1}{2} p\left(\gamma_{E}-2\right)+\frac{1}{2}\left(\frac{T^{2}}{3}-\frac{T}{\sqrt{\pi^{3} \delta}}\right) H_{1} \\
& +\frac{1}{48 \pi^{2}}\left\{-\frac{11}{4}\left[\gamma_{E}-3.1+\ln \left(\frac{\Lambda^{2}}{4 T^{2}}\right)\right] F_{2}-G_{2}+D_{2}-2 H_{2}\right\}
\end{aligned}
$$

\subsubsection{Renormalization}

It is worth noticing that the last term on the first line of (2.3.13) cancelled the PauliVillars ultraviolet regulator $\left(\Lambda^{2}\right)$ in the prefactor of eqn. (2.2.20) and replaced it by an infrared cutoff mass $(1 / \delta)$ in eqn. (2.3.16). One might at first suspect this as being an anomalous artifact of our derivative expansion, but it is worth noting that in 't Hooft's exact one loop instanton calculation, his ultraviolet regulator in the prefactor was also replaced by an infrared scale - the size of the instanton $(\rho)$. Moreover, renormalization can always be performed by using counterterms in the original Lagrangian which have the same symmetry as that of the background field at zero temperature. Although the gauge of the background field has been fixed, it has not been specified; consequently, the counterterms must take the form $C F_{\mu \nu}^{2}$, where $C$ is some constant depending on $\Lambda^{2}$. It is therefore reassuring that the only $\Lambda^{2}$ dependence comes in the coefficient of a term multiplying $F_{2}$, so that all ultraviolet divergences can be removed by normal counterterms.

Because the counterterms in the background gauge have the same form as the original Lagrangian, one can create a renormalized Lagrangian simply by multiplying the original bare Lagrangian by the factor:

$$
Z_{3}=1-\frac{11 N g^{2}}{18 \pi^{2}} \ln \left(\frac{Q^{2}}{\Lambda^{2}}\right)
$$

where $g$ now represents the running coupling. At very high temperature, it is most convenient to choose the renormalization scale to be $Q^{2} \simeq 4 T^{2}$ ex $\left(3.1-\gamma_{E}\right) \sim 50 T^{2}$ 
in order to absorb all of the one loop coefficients of $F_{2}$ into the definition of the renormalized (running) coupling. This running coupling constant is then defined in terms of the bare coupling by:

$$
\frac{1}{g^{2}}\left[1-\frac{11 g^{2} N}{48 \pi^{2}}\left[3.1-\gamma_{E}+\ln \left(\frac{4 T^{2}}{\Lambda^{2}}\right)\right]=\frac{1}{g_{0}^{2}} .\right.
$$

Just as at zero temperature, the running coupling can be defined in terms of an experimentally determined mass scale[Fie89]. We will denote this scale by $\Lambda_{\mathrm{QCD}}$ even for theories other than QCD. The running coupling can then be expressed

$$
\frac{g^{2}}{4 \pi}=\frac{12 \pi}{11 N \ln \left(T^{2} / \Lambda_{\mathrm{QCD}}^{2}\right)},
$$

and the renormalized effective action takes the form:

$$
S_{\mathrm{eff}}=\frac{1}{4 N g^{2}} F_{2}+\frac{1}{2} p\left(2-\gamma_{E}\right)+\frac{1}{2}\left(\frac{T^{2}}{3}-\frac{T}{\sqrt{\pi^{3} \delta}}\right) H_{1}+\frac{1}{48 \pi^{2}}\left\{D_{2}-G_{2}-2 H_{2}\right\}
$$

If a different renormalization scale $Q^{2}$ is chosen, the coefficient of $F_{2}$ in (2.3.21) will be altered by an $O(1)$ term, and $\Lambda_{\mathrm{QCD}}$ in (2.3.20) will be multiplied by a calculable factor.

\subsubsection{Constant Background $A_{0}$ Field}

To test the accuracy of this covariant derivative expansion, we can plug in a constant $\mathrm{SU}(2)$ background field of $A_{0}^{3}=\eta$, with all other field components vanishing. There are no non-gauge zero modes in this configuration, so $p=0$. It can also be shown that $D_{2}=F_{2}=G_{n}=0$ and that

$$
H_{2}=-(g \eta)^{2} H_{1}=2(g \eta)^{4} V / T,
$$

where $V=\int d^{3} x$ is an infinite spatial volume. The infrared cutoffs and effective action from eqns. (2.3.15) and (2.3.21) take the simple forms:

$$
\begin{aligned}
\delta & =\frac{2}{(g \eta)^{2}} \\
S_{\text {eff }} & =-\frac{V}{T}\left[\frac{T^{2}}{3}(g \eta)^{2}-\frac{T}{\sqrt{2 \pi^{3}}}(g \eta)^{3}+\frac{1}{12 \pi^{2}}(g \eta)^{4}\right] .
\end{aligned}
$$




\section{SU(2) Effective Potential}

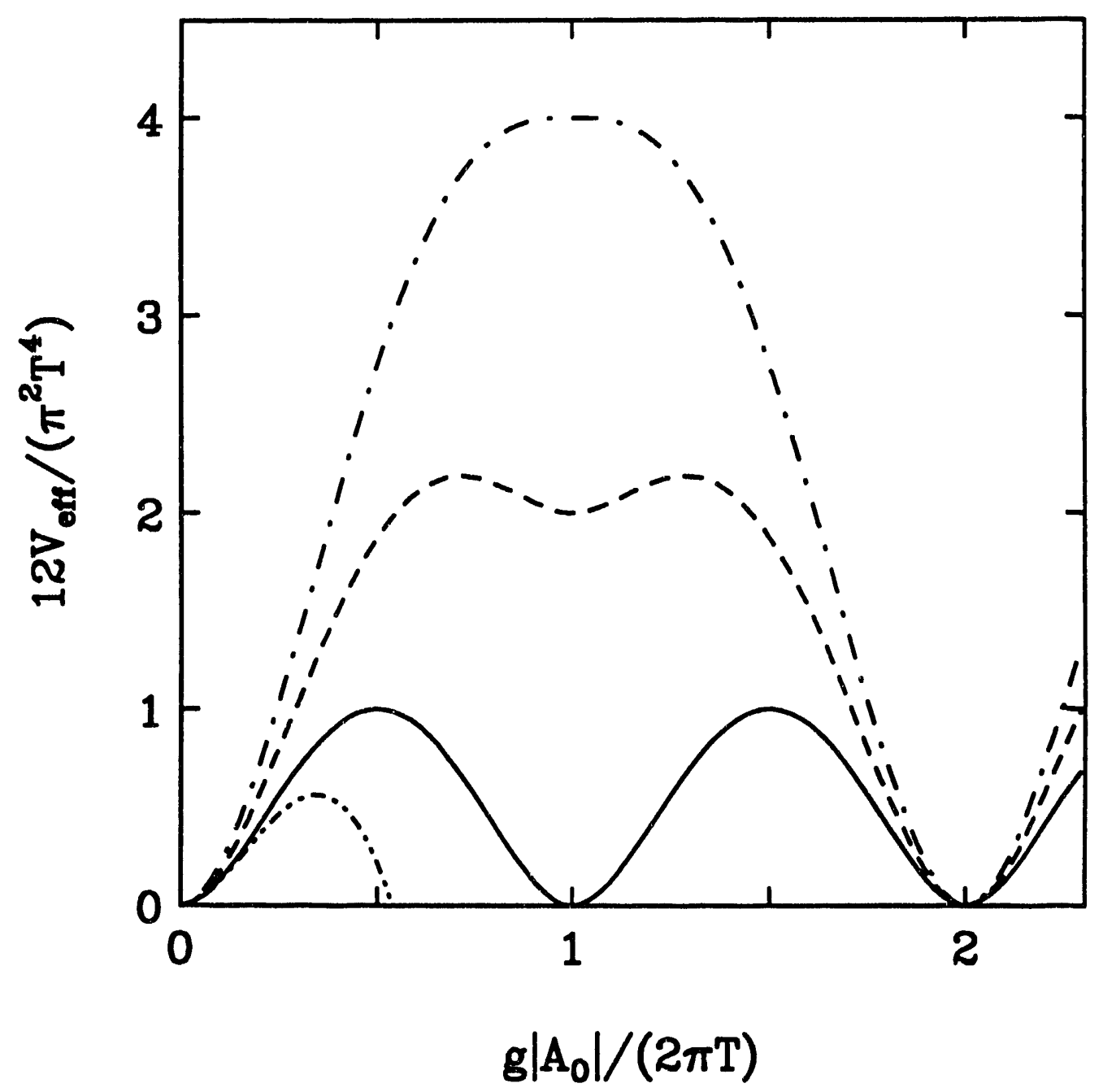

Figure 2.3.1 The one loop SU(2) $A_{0}$ effective potential with no fermions (solid), 1 massless fermion (dashed), and 2 fermions (dot-dashed). The dot-dot-dashed curve shows the lowest order covariant derivative expansion result of eqn. (2.3.23).

The exact answer is well-known to be (see Appendix B):

$$
S_{\text {eff }}=-\frac{V}{T} V_{\text {eff }}(\eta)=-\frac{V}{T}\left[\frac{T^{2}}{3}(g \eta)^{2}-\frac{T}{3 \pi}(g \eta)^{3}+\frac{1}{12 \pi^{2}}(g \eta)^{4}\right]_{\bmod 2 \pi \mathrm{T}} .
$$

where mod $2 \pi T$ applies to each factor of $g \eta$ in $S_{\text {eff. }} V_{\text {eff }}$ is plotted in figure 2.3 .1 . It is apparent that no finite number of terms in the derivative expansion outlined above 
will be able to produce a periodic effective potential for the $A_{0}$ field. Nevertheless, if one is interested in field configurations for which $A_{0} \sim \mathcal{O}\left(g^{\alpha} T\right)$ with $\alpha>-1$ in the $T \rightarrow \infty$ limit, then only the quadratic term in the effective potential will be important. Since the derivative expansion correctly reproduces this term (fig. 2.3.1), it is reasonable to use the expansion to describe the above class of configurations. It is important to note on the other hand that the derivative expansion is a bad approximation for configurations with $\alpha=-1$ even when $T \rightarrow \infty$, because in this case the cubic term and periodic nature of $V_{\text {eff }}$ become important. For example, one should not use this expansion to study configurations in which the $A_{0}$ field approaches one of the minima at $2 n \pi T / g$.

\subsection{Application to Dyons}

It has been suggested[Polo91] that a plasma of magnetically charged solitons featuring $A_{0} \rightarrow \mathcal{O}(g T)$ as $r \rightarrow \infty$ could possibly self-stabilize in the $T \rightarrow \infty$ limit of $\mathrm{SU}(2)$, even though there is no $\mathcal{O}(g T)$ minimum in the $A_{0}$ effective potential. The derivative expansion can be used to study this idea more carefully. We make the following ansatz for spherically symmetric soliton configurations:

$$
A_{0}^{a}=\eta \frac{r_{a}}{r} f(x) \quad A_{i}^{a}=\eta \epsilon_{i a j} \frac{r_{j}}{r} h(x),
$$

where $\eta$ is the expectation value of $\left|A_{0}\right|$ at infinity and $x=g \eta r$. With this ansatz, the equations of motion (2.2.4) take the dimensionless form[Bia76]:

$$
\begin{aligned}
x^{2} f^{\prime \prime}+2 x f^{\prime}-2 f(1+x h)^{2} & =0 \\
x^{2} h^{\prime \prime}+2 x h^{\prime}-(1+x h)\left(2 h+x h^{2}+x f^{2}\right) & =0,
\end{aligned}
$$

where the primes denote derivatives with respect to $x$. Since $f, h$ and $x=g \eta r$ are dimensionless, any solution of the above equations will have a characteristic length scale of $\mathcal{O}(1 / g \eta)$. 
Note that in ansatz (2.4.1), the magnitude of the $A_{0}$ field approaches a nonzero çnstant value as $r \rightarrow \infty$. Rather than compare such configurations to the perturbative $A_{0}=0$ vacuum, it is more useful to compare them to a background with a constant $\left|A_{0}\right|=\eta$ field. From the form of $V_{\text {eff }}$ in eqn. (2.3.24), it is apparent that such a background has infinitely more free energy (by a volume factor) than the perturbative vacuum, but it is possible that the infinite increase of entropy gained by introducing a plasma of solitons will offset the infinite background energy and allow such a plasma to self-stabilize. In other words, we would like to determine whether the free energy of a plasma of dyons in a constant background field is lower than that of the perturbative $A_{0}=0$ vacuum. To do this, we must calculate $Z / Z_{\eta}$, where $Z$ is the partition function for a background dyon configuration and $Z_{\eta}$ is that for a constant $\left|A_{0}\right|=\eta$ background field. All of our previous calculations have been for $Z / Z_{0}$ where $Z_{0}$ refers to the perturbative vacuum, so some of our expressions must be modified. Fortunately, $F_{2}=D_{2}=G_{n}=0$ for both a constant field and the perturbative vacuum, so only the $H_{n}$ are different. In fact all of the necessary modifications can be made by simply subtracting from each $H_{n}$ the value of $H_{n}$ for a constant field:

$$
H_{n} \rightarrow H_{n}-2\left(-g^{2} \eta^{2}\right)^{n} V / T
$$

Much of our discussion will center around the Prasad-Sommerfield-Julia-Zee (PSJZ) dyon, which is a magnetic and electrically charged self-dual solution of the classical Euclidean SU(2) Lagrangian for any value of $\eta$. It is defined by:

$$
f(x)= \pm(\operatorname{coth}(x)-1 / x) \quad h(x)= \pm(\operatorname{csch}(x)-1 / x)
$$

where the \pm reflects the fact that both dyons and antidyons are solutions to the equations of motion, each having a tree level action given by $\bar{S}=-4 \pi \eta / g T$ [Act79].

In addition to three translational zero modes which were treated previously as an example, these dyons each have a global gauge zero mode which is not eliminated by the hackground gauge requirement[Mot78]. To find the prefactor associated with this zero mode, it is best to consider the monopole in the string gauge. In this gauge, 
$A_{0}^{a}=\delta^{a 3} \eta f$, and the $A_{i}^{a}$ field has a Dirac string singularity along the $-\hat{z}$ axis. The string gauge form of the solution can be obtained from the spherically symmetric form by making a gauge transformation with the following gauge function[Col81]:

$$
U(\theta, \phi)=\exp \left(i \sigma_{3} \phi / 2\right) \exp \left(i \sigma_{2} \theta / 2\right) \exp \left(-i \sigma_{3} \phi / 2\right)
$$

where $\sigma_{a}$ are the Pauli matrices.

Consider the following global gauge transformation:

$$
A_{\mu} \rightarrow A_{\mu}^{\prime}=G A_{\mu} G^{-1}
$$

where $A_{\mu}=\sigma_{a} A_{\mu}^{a}$ and $\mathrm{G}$ is given by:

$$
G=\exp \left(i \sigma_{3} \omega g \eta / 2\right)
$$

Treating $\omega$ as an infinitessimal collective coordinate, we find:

$$
\frac{\partial A_{\mu}^{\prime}}{\partial \omega}=-\frac{1}{2} i g \eta\left[A_{\mu}, \sigma_{3}\right]=\sigma_{a} D_{\mu}^{a b}\left(\delta_{b 3} \eta\right)
$$

By making a careful choice of the gauge function $\theta_{a}$ from eqn. (2.2.10), we get the following zero mode:

$$
\chi_{\mu}^{a}=\frac{\partial A_{\mu}^{a \prime}}{\partial \omega}+D_{\mu}^{a b}\left[\eta \delta_{b 3}(f-1)\right]=F_{\mu 0}^{a}
$$

which satisfies the background gauge requirement (2.2.6) through the equations of motion (2.2.4). Like the translational modes, the normalization of this mode is $N_{0}=$ $-\bar{S}$, and the partition function involves an integral over the collective coordinate $\omega$. However, unlike the translational modes, $\omega$ has a finite range of $0<\omega<4 \pi / g \eta$, as can be easily seen by examining the form of $G$ in eqn. (2.4.7). The entire prefactor for the dyon can now be expressed in terms of the infrared cutoff $\delta$ :

$$
\xi=\frac{Z}{Z_{\eta}}=\frac{16 \pi \eta V}{g^{3} T^{2} \delta^{2}} \exp \left(S_{\text {eff }}\right)
$$

where $V$ is the volume of space and $S_{\text {eff }}$ is defined by eqn. (2.3.24) with the replacement (2.4.3). 
As we mentioned in the Introduction, if we assume that identical dyons are noninteracting, then we can approximate the one loop functional integral around two well separated dyons by $\xi^{2} / 2$. The factor of $1 / 2$ is included in order to avoid double counting when the positions of the identical dyons are switched. Similarly, for a solution with $N$ identical dyons, there will be a symmetry factor of $1 / N$ !. A full one loop calculation of the partition function should incorporate quadratic fluctuations around every single saddle point of the original Lagrangian which has the same boundary conditions at infinity. If we demand that $A_{0}^{3} \rightarrow \eta$ as $r \rightarrow \infty$, then the saddle points include any number of dyons and antidyons, as well as a constant background field with no dyons:

$$
Z / Z_{0}=Z_{\eta} / Z_{0} \exp (2 \xi)=\exp \left(-V_{\text {eff }}(\eta) V / T+2 \xi\right)
$$

where the factor of 2 reflects the sum over both dyon and antidyon saddle points, and $Z_{0}$ is the partition function of the perturbative vacuum. Using $V_{\text {eff }}(\eta)$ from (2.3.24) and dropping all but the quadratic term, we get the following expression for the free energy density of a dyon plasma compared to that of the pers trative vacuum:

$$
\Omega=-(T / V) \ln Z=\frac{1}{3}(g \eta T)^{2}-\frac{32 \pi \eta}{g^{3} T \delta^{2}} \exp \left(\hat{\kappa}_{\mathrm{eff}}\right) .
$$

The trick of self-stabilization as $T \rightarrow \infty$ is to see if a minimum of $\Omega$ can be found for some nonzero value of $\eta$.

For the moment, let us assume that as $T \cdots(g \rightarrow 0)$ one loop corrections are parametrically smaller than the tree level ation (i.e. we assume that infrared divergences do not destroy this property). We can therefore replace $S_{\text {eff }}$ in (2.4.12) by $\bar{S}=-4 \pi \eta / g T$. Because of the exponential dependence of the second term, we can see that the only hope of finding a nontrivial minimum would be for $\eta \sim \mathcal{O}\left(g^{\alpha} T\right)$ with $\alpha \geq 1$. Furthermore, the prefactor of the second term could be of no higher order in $g$ than $g^{2+2 \alpha}$ since that would be the order of the first term. From the discussion in the Introduction, we can see that the density of the plasma would be $\sim \mathcal{O}\left(g^{2+2 \alpha} T^{3}\right)$, while from the discussion after eqn. (2.4.2), we know that the size of a 
dyon is $\sim \mathcal{O}\left(1 / g^{1+\alpha} T\right)$. In other words, for $\alpha \geq 1$ the dyons would have to be strongly overlapping. Furthermore, since the difference in length scales is a parametric one, the overlapping would get infinitely worse as $g \rightarrow 0$.

Is this really a problem? If the plasma was comprised only of identical dyons with no antidyons, then overlapping might not be a problem since topologically stable, overlapping dyon solutions which are classically noninteracting have already been found[Cor81]. On the other hand, a dyon and an antidyon can annihilate, so the approximation that we have been using that they are noninteracting would be a very bad one for a strongly overlapping plasma of dyons and antidyons. If an overlapping neutral plasma did in fact exist, it would have to be strongly interacting and consequently very difficult to describe using semi-classical methods. Furthermore, as Gross et al.[Gro81] pointed out when making a similar argument about a plasma of Wu-Yang monopoles, such a plasma, with typical field strengths on the order of $g T$, would be difficult to distinguish from normal fluctuations around the perturbative vacuum. Perhaps the only clue to its existence might be the enhancement of low frequency fluctuations[Man76]. In order to avoid the problem of annihilation, it has been suggested that some mechanism could be found which would stabilize large domains of dyons and antidyons[Polo92]. Even with such a mechanism, the fact that each dyon has zero field strength at the origin would still make a parametrically overlapping plasma domain locally very difficult to distinguish from the perturbative vacuum.

It is interesting to see what value of $\alpha$ would be necessary to make a plasma of dyons nonoverlapping in the $g \rightarrow 0$ limit. Suppose that infrared divergences in one loop terms miraculously caused them to be of the same order as tree level terms and were able to render $S_{\text {eff }} \sim \mathcal{O}(1)$, even when $\eta \sim \mathcal{O}(T)$. The prefactor of the second term in eqn. (2.4.12) would then have to be at most $\mathcal{O}\left(g^{2}\right)$ in order to create a nontrivial minimum. In such a scenario, the typical separation would be $\sim 1 / g^{2 / 3} T$ while the size of a dyon would be $\sim 1 / g T$. Again, the plasma would 
be parametrically overlapping in the $g \rightarrow 0$ limit. Using similar reasoning, it can be shown that the only hope of crei ting a self-stabilized, nonoverlapping plasma of dyons would be for $\alpha \leq-1$, which is exactly the range of $\alpha$ for which the covariant derivative expansion becomes unreliable. We can therefore conclude that no weakly interacting, nonoverlapping plasma of Prasad-Sommerfield dyons with $\alpha>-1$ will be able to self-stabilize in the $g \rightarrow 0$ limit.

I would like to make a couple of remarks before continuing. It has been suggested that by using a Coleman-Weinberg type mechanism[Col73] to minimize the effective action rather than the classical action, one may be able to to find monopole solutions with $A_{0} \rightarrow \mathcal{O}(g T)[$ Polo91]. The idea would be that after combining the one loop effective potential with the tree Lagrangian, solutions could be found for which $A_{i}$ drops off like $1 / x$ at large distances, but $A_{0}$ only approaches $\eta$ like $\exp (-C x)$. Such a solution would not have a long range electric field and would consequently be a magnetic monopole rather than a dyon. As we shall show later however, in order to find such a monopole, it is necessary that the $A_{0}$ field approaches a local minimum of the effective potential as $r \rightarrow \infty$ (see section 7 ). Unfortunately, no evidence has been found for such a minimum[Bel91], except for the periodic minima at $2 n \pi T / g$ mentioned earlier. It is still possible that a plasma of Wu-Yang-type monopoles as suggested in [Gro81] or a strongly interacting plasma of dyons could provide a magnetic screening mass of $\sim \mathcal{O}\left(g^{2} T\right)$ as $g \rightarrow 0$, but if so, it is not clear that semiclassical methods would be useful in describing these effects. On the other hand, it would be interesting to see whether the situation changes at all for dyons with $\eta \sim \mathcal{O}(T / g)$. To do so, we must perform some infinite resummations which will improve our covariant derivative expansion. 


\subsection{Improved Expansion}

The periodicity of $V_{\text {eff }}$ in eqn. (2.3.24) is simply a consequence of invariance under temporal gauge transformations. To see this, we first note that due to unitarity and the temporal boundary conditions at finite temperature, the most general gauge transformation for pure gauge $\mathrm{SU}(2)$ (see appendix $\mathrm{C}$ ) is given by:

$$
U(\tau, \vec{x})=\exp \left\{i \sigma_{a}\left[\theta^{a}(\tau, \vec{x})+n_{a} \pi T \tau\right]\right\},
$$

where $\theta^{a}$ is periodic in $\tau, n_{a}$ are integers, and $\sigma_{a}$ are the Pauli matrices. Since the gauge of the background field is never specified in the background field formalism, any effective potential for the $A_{0}$ field must be gauge independent. The most general gauge invariant expressions involving $A_{0}$ but not $A_{i}$ are integral powers of the Polyakov line

$$
\operatorname{tr} \exp \left[i g n \sigma_{a} \int_{0}^{\beta} A_{0}^{a} d \tau\right] .
$$

Thus the most general possible gauge invariant expression for the pure gauge SU(2) $A_{0}$ effective potential is:

$$
V_{\text {eff }}\left(A_{0}\right)=\sum_{n=0}^{\infty} a_{n} \cos \left(g n \sigma_{a} \int_{0}^{\beta} A_{0}^{a} d \tau\right) .
$$

Because the above effective potential is a general expression which should hold for any field configuration, our knowledge of the exact answer for a constant $A_{0}$ field uniquely determines the coefficients $a_{n}$ in the one loop approximation. For SU(2), we have:

$$
a_{0}=\frac{2 \pi^{2} T^{4}}{45} \quad a_{n>0}=-\frac{4 T^{4}}{\pi^{2} n^{4}}
$$

which leads to the correct expression (2.3.24) for $A_{0}^{3}=\eta$.

Since the form of eqn. (2.5.2) is only a result of gauge invariance, $V_{\text {eff }}$ for all consistent higher order calculations must also take that form, though the coefficients will of course be modified. As a consequence, $V_{\text {eff }}$ can never feature a linear term at the origin (i.e. $A_{0}=0$ must always be an extremum of the potential). This is significant, since if one makes a two loop calculation of the effective potential, a 
linear term does appear which seems to create a minimum of $\mathcal{O}(g T)$ in the effective potential[Ani84, Dah85]. From the above arguments, however, we know that such a linear term is spurious and must vanish in a consistent $\mathcal{O}\left(g^{n}\right)$ calculation (which does not always coincide with a loop expansion). It is therefore not surprising that summing the infrared divergent Debye ring diagrams (with more than two loops) in $\mathrm{SU}(2)$ gives rise to a linear term which exactly cancels the one found at the two loop order[Bel91].

Where is $V_{\text {eff }}$ hidden in our covariant derivative expansion? The main problem with our expansion is that we are expanding a gauge invariant effective action in terms of functionals like $H_{n}$ and $G_{n}$ which are gauge dependent. Nevertheless, if we had had the patience and fortitude to calculate all terms in the expansion out to infinite order, making no approximations and letting $\delta \rightarrow \infty$, we would have arrived at an exact and gauge invariant expression for the effective action. In particular for $\mathrm{SU}(2)$, all of the terms $H_{n}$ would have summed up to form the effective potential of eqns. (2.5.2) and (2.5.3). We can therefore improve our approximation of $S_{\text {eff }}$ by including the known form of $V_{\text {eff }}$ and dropping all $H_{n}$ terms. By construction, our effective action will then exactly reproduce $V_{\text {eff }}(\eta)$ from eqn.(2.3.24).

After having resummed the $H_{n}$ terms, the only remaining gauge dependent terms are $G_{n}$. The main problem with these terms is that they do not reflect the equivalence between configurations with $A_{0}$ near the different minima at $2 n \pi T / g$. We can solve this problem by introducing new functionals $G_{n}^{\prime}$ which do reflect that equivalence:

$$
G_{n+1}^{\prime}=-2 T^{2} \operatorname{tr} \int d^{4} x\left[D_{i},\left[D_{i}, \cos \left(n D_{0} / T\right)\right]\right]
$$

In particular, for static $\mathrm{SU}(2)$ fields with $g\left|A_{0}\right|<<T$, we get:

$$
G_{2}=G_{2}^{\prime}=-\frac{2 g^{2}}{T} \int d^{3} x \partial_{i}^{2} A_{0}^{2}
$$

Thus to the order that we are working, if we replace $G_{2}$ by $G_{2}^{\prime}$, we not only reproduce the correct behavior for static fields with small magnitudes, we also introduce the periodicity necessary to describe configurations with $\left|A_{0}\right|$ near each of the minima at 
$2 n \pi T$. If we wanted to take the derivative expansion to the next order, we would get some terms involving $G_{3}$. We could then replace $G_{2}$ and $G_{3}$ by their primed counterparts, choosing coefficients such that the behavior of static fields with $g\left|A_{0}\right|<<T$ was not altered. In addition, new gauge dependent terms involving more derivatives of $D_{0}^{2}$ could be replaced by terms having the same small $\left|A_{0}\right|$ behavior, but which reflect the equivalence of the $A_{0}$ minima. In this way, a modified covariant derivative expansion for static $\mathrm{SU}(2)$ fields can be continued to higher orders with the gauge equivalence of the $A_{0}$ minima manifest at each step.

Looking back at eqn. (2.3.15), we can see that after resumming the $H_{n}$ terms, the new $\delta$ is given by:

$$
\sqrt{\delta}=\frac{48 \sqrt{\pi^{3}} p}{T\left(-11 F_{2}+2 G_{2}^{\prime}-2 D_{2}\right)}
$$

Notice that $\delta=0$ for configurations without zero modes. This just means that for these configurations, we would need to keep more terms in the derivative expansion to get a reliable value for $\delta$. However, since we are primarily interested in calculating prefactors for configurations with zero modes, the above definition of $\delta$ is sufficient provided that it turns out that $\delta>0$. Assuming this, we can write down a partially resummed, renormalized effective action for pure gauge $\mathrm{SU}(2)$ :

$$
S_{\mathrm{eff}}=\frac{1}{4 N g^{2}} F_{2}-\frac{1}{2} p\left(\gamma_{E}-2\right)+\frac{1}{48 \pi^{2}}\left(D_{2}-G_{2}^{\prime}\right)-\frac{1}{T} \int d^{3} x V_{\mathrm{eff}}\left(A_{0}\right)
$$

where $V_{\text {eff }}$ is given by eqns. (2.5.2) and (2.5.3). Equations (2.5.6) and (2.5.7) along with eqn. (2.3.16) are the main results of this paper.

\subsection{More Dyons}

We would now like to apply our improved formalism to the case of a PSJZ dyon for which $\left|A_{0}\right| \rightarrow \eta=2 \pi T / g$ as $r \rightarrow \infty$. Since $A_{0}$ approaches one of the absolute minima of the effective potential at infinity, a plasma of these dyons would not have to "self-stabilize" its entropy against an infinite background energy, as was the case 
of the dyons considered previously. Since the PSJZ dyon is self dual,

$$
F_{2}=4 D_{2}=8 g^{2} \bar{S}=-64 \pi^{2}
$$

The integral for $G_{2}^{\prime}$ is convergent and can be found to be:

$$
G_{2}^{\prime}=-16 \pi^{2}
$$

Since the dyon has four zero modes, the infrared cutoff can be found from (2.5.6) to be:

$$
\delta=\frac{1}{\pi}\left(\frac{3}{11 T}\right)^{2}
$$

Note that $\delta \sim \mathcal{O}\left(1 /(g \eta)^{2}\right)$ just as it was for a constant $A_{0}$ field. Keeping more terms in the derivative expansion will not affect the order of $\delta$, though it will affect the size of the $\mathcal{O}(1)$ coefficient. Looking at eqn. (2.4.10), we can see that the entire plasma prefactor is determined, and we only need to evaluate $S_{\text {eff }}$ in order to determine the density of the plasma.

Here is where we run into problems. We might at first think that we can simply replace $S_{\text {eff }}$ by $\bar{S}$ in the exponent of (2.4.10) because the one loop corrections $S_{\text {eff }}^{(1)}$ are down by $\mathcal{O}\left(g^{2}\right)$. However, the fact is that for an isolated dyon, $S_{\text {eff }}^{(1)}$ diverges like a distance at infinity since $A_{0}$ only approaches the minimum at $2 \pi T / g$ like $1 / x$. We can see this by cutting off the integral over $V_{\text {eff }}$ at some large radius $R$ :

$$
\frac{4 \pi}{T} \int_{0}^{R} r^{2} d r V_{\text {eff }}\left(A_{0}\right) \simeq 8 \pi^{2}(2 \pi T R)
$$

For a neutral plasma, we could argue that the highest electric multipole moment at infinity would be a dipole and so this divergence would not really occur. Let us assume that this is the case and try to find some sensible procedure for estimating $R$ in the $g \rightarrow 0$ limit. The simplest guess would be that $2 \pi T R \sim \mathcal{O}\left(g^{-\alpha}\right)$. For any positive $\alpha$, $R$ would be parametrically larger than the typical size of a dyon $\sim \mathcal{O}(1 /(2 \pi T))$. On the other hand, as long as : $<2, \bar{S}$ will dominate $S_{\text {eff }}$ and the density of the plasma can be found from $(2.4 .10)$ to be $\mathcal{O}\left(g^{-4} \exp \left(--8 \pi^{2} / g^{2}\right) T^{3}\right)$. In the $g \rightarrow 0$ limit, one 
would expect $R$ to be of the same order in $g$ as the typical separation between dyons, but we can see that due to the exponential dependence of the density on $1 / g^{2}$, this cannot be achieved in the $g \rightarrow 0$ limit. In fact, trying to find an equivalence between $R$ and the typical dyon separation will drive $R \rightarrow \infty$ in the $g \rightarrow 0$ limit. Thus, due to one loop effects, PSJZ dyons with $\eta=2 \pi T / g$ will become infinitely heavy and decouple from the theory as $T \rightarrow \infty$.

On the other hand, we should not dismiss these dyons so easily for finite temperatures, in particular when $T \rightarrow \Lambda_{Q C D}$. For a neutral plasma at finite $T$, it might be that a scale could be found for $R$ which would be in qualitative agreement with the typical dyon separation which we will hereafter call $R_{s}$. In other words, we would like to find an $\mathrm{R}$ for which:

$$
R \simeq \frac{1}{2} R_{s}=\frac{1}{2}\left(\frac{3}{4 \pi V} \frac{Z}{Z_{\eta}}\right)^{-1 / 3},
$$

where $R_{s}$ depends on $R$ through $S_{\text {eff }}$ and $Z / Z_{\eta}$ is given by eqns. (2.4.10) and (2.4.9). It turns out that this equation only starts having solutions for $g>4$. Obviously at this point, we have left the regime of weak coupling, so the one loop approximation becomes dubious at best. In addition, it can be shown that the $R$ 's which solve (2.6.5) are typically between $1 /(4 \pi T)$ and $1 /(2 \pi T)$ which is the same scale as the size of the dyon, so dyons and antidyons would again begin to overlap.

\subsection{Monopoles}

One way that we could dispose of the troublesome divergence of $\int d^{3} x V_{\text {eff }}$ would be if we could find a way to make $A_{0}$ approach $2 \pi T / g$ faster than $1 / x$. In the 't HooftPolyakov monopole, the Higgs field approaches its vacuum expectation value like $\exp (-M x)$, which is just a consequence of it going to a quadratic minimum. If we use the Coleman-Weinberg mechanism[Col73] to find configurations which minimize $S_{\text {eff }}$ rather than classical solutions which minimize $\bar{S}$, we should be able to achieve the desired behavior for $A_{0} \rightarrow 2 \pi T / g$ as $r \rightarrow \infty$ since there is a quadratic minimum in the 
effective potential there. To really use the Coleman-Weinberg mechanism with a clear conscience, we should include all orders of the derivative expansion in our expression for $S_{\text {eff }}$ before we minimize, and we should verify that the configurations that we are interested in have no negative eigenmodes associated with them. Nevertheless, we shall proceed in the most naive manner, keeping only the effective potential and not worrying about negative eigenmodes for the time being.

For $r \rightarrow \infty$, the extrema of $S_{\text {eff }}$ can be found by solving the following equations:

$$
D_{\mu}^{a b} F_{\mu \nu}^{b}-\delta_{\nu 0} \frac{\partial V_{\text {eff }}}{\partial A_{0}^{a}}=0
$$

These equations are greatly simplified by using the ansatz (2.4.1) along with the definition:

$$
f(x)=1+\frac{F(x)}{x} \quad h(x)=\frac{H(x)-1}{x} .
$$

Equations (2.7.1) then become[Act79]:

$$
\begin{aligned}
& x^{2} H^{\prime \prime}=H\left(H^{2}-1+(x+F)^{2}\right) \\
& x^{2} F^{\prime \prime}=2(x+F) H^{2}+\frac{1}{3 \pi^{2}} F\left(F+\frac{1}{2} x\right)(F+x),
\end{aligned}
$$

where the primes denote derivatives with respect to the variable $x=g \eta r$, and we have assumed that $\eta=2 \pi T / g$. For a monopole configuration, the $A_{i}$ fields should drop off like $-1 / x$ far from the origin. From the definitions of (2.7.2), then, we expect $H$ and $F$ to be small as $x \rightarrow \infty$. In this limit, the equations of motion become $H^{\prime \prime}=H$ and $F^{\prime \prime}=F / 6 \pi^{2}$, so that:

$$
H \rightarrow C_{1} \exp (-x) \quad F \rightarrow C_{2} \exp \left(-\frac{x}{\sqrt{6 \pi^{2}}}\right)
$$

If we try to find a monopole for which $A_{0}$ asymptotically approaches a value which is not a minimum, then we find an equation like $F^{\prime \prime}=C x$, which does not feature solutions which vanish as $x \rightarrow \infty$. We can conclude that only monopole configurations in which the $A_{0}$ field approaches a minimum of the effective potential have any chance of minimizing the effective action. 


\subsubsection{Negative Modes}

Unfortunately, in deriving $S_{\text {eff }}$ for the monopole, we have implicitly integrated over negative eigenmodes. To see this, let us look a little more closely at what it means to integrate around a configuration which minimizes the effective action rather than the classical action. Suppose we have a monopole configuration $\bar{A}_{\mu}^{a}$ defined by eqns. (2.4.1), (2.7.2) and (2.7.5). Since $\bar{A}_{\mu}^{a}$ is not a classical solution, when we make the replacement $A_{\mu}^{a}=\bar{A}_{\mu}^{a}+B_{\mu}^{a}$ there will be terms linear in $B_{\mu}^{a}$. Nevertheless, by adding an appropriate current term $J_{\mu}^{a} A_{\mu}^{a}$ to the original Lagrangian, the linear terms can be exactly cancelled and the monopole configuration becomes a solution to the modified equations of motion:

$$
D_{\mu}^{a b} F_{\mu \nu}^{b}=J_{\mu}^{a} .
$$

It is now possible to perform gaussian functional integrals over the terms which are quadratic in $B_{\mu}^{a}$ as long as none of the operators involved have negative eigenmodes (i.e. the configuration is stable). If, on the other hand, there are negative eigenmodes, then some of our "gaussian" functional integrals would actually be integrals of the type $\int \exp \left(+\alpha x^{2}\right) d x$ which diverge and render the one loop approximation useless. In the absense of negative modes, the current $J$ is set equal to zero at the one loop level if the original configuration turns out to be an extremum of the effective action[Col73]. In a sense, we have gone about things a bit backwards by first finding a configuration which sets $J=0$. We must now go back and check whether or not the configuration was classically stable to begin with.

Far from the center of the monopole, exponentially falling functions are unimportant, so we can approximate the configuration by using (2.4.1) and (2.7.2) with $H=F=0$. We can then find an explicit expression for the operator inside the ghost determinant:

$$
-D^{2}=\left[-i \partial_{0}-2 \pi T(I \cdot \hat{r})\right]^{2}-\frac{\partial^{2}}{\partial r^{2}}-\frac{2}{r} \frac{\partial}{\partial r}+\frac{1}{r^{2}}\left[J^{2}-(I \cdot \hat{r})^{2}\right]
$$

where $I, L$ and $J$ are isospin, orbital, and total angular momentum operators given 
by:

$$
\begin{aligned}
\left(I_{c}\right)^{a b} & =-i \epsilon^{a b c} \\
L_{i} & =-i \epsilon_{i j k} r_{j} \partial_{k} \\
J & =I+L .
\end{aligned}
$$

We are interested to see whether this operator has any negative eigenvalues. For static configurations, we can use temporal eigenfunctions of $\exp (i 2 n \pi T \tau)$ and see that the first term of $-D^{2}$ is positive semi-definite by making the replacement $-i \partial_{0} \rightarrow 2 n \pi T$. In addition, we can see that the last term is positive definite by noting that $(L \cdot \hat{r})=0$ and replacing $(I \cdot \hat{r})$ by $(J \cdot \hat{r})$. Furthermore, the radial derivative terms are positive definite since

$$
-\partial_{i}^{2}=-\frac{\partial^{2}}{\partial r^{2}}-\frac{2}{r} \frac{\partial}{\partial r}+\frac{L^{2}}{r^{2}}
$$

is positive definite even when $L^{2}=0$. Therefore the whole ghost operater is positive definite.

What about the gauge operator? To begin examining $W$, we first note that far from the monopole, there is no electric field and consequently $F_{0 i}^{a}=0$. From eqn. (2.2.7), this implies that $W_{0 i}=W_{i 0}=0$. The gauge determinant can then be separated into two determinants:

$$
\operatorname{det}\left(W_{\mu \nu}\right)=\operatorname{det}\left(-D^{2}\right) \operatorname{det}\left(W_{i j}\right)
$$

where we have already shown that the first is positive definite. Dropping the spatial indices on $W_{i j}$, we can use techniques similar to those used for the ghosts to write:

$$
W=(2 \pi T)^{2}(n-(I \cdot \hat{r}))^{2}-\frac{\partial^{2}}{\partial r^{2}}-\frac{2}{r} \frac{\partial}{\partial r}+\frac{1}{r^{2}}\left\{K^{2}+S^{2}-2 S \cdot[K-\hat{r}(I \cdot \hat{r})]-(I \cdot \hat{r})^{2}\right\}
$$

where $S$ and $K$ are spin and total angular momenta defined by:

$$
\begin{aligned}
\left(S_{k}\right)^{i j} & =-i \epsilon_{i j k} \\
K & =I+S+L .
\end{aligned}
$$


The only nonzero commutator among the operators of $(2.7 .11)$ is between $S \cdot K$ and $S \cdot \hat{r}$. Even with this difficuly, however, we can still make $W$ block diagonal by quantizing with respect to $S^{2}, K^{2}, m=(I \cdot \hat{r}), s=(S \cdot \hat{r})$ and $l=K_{z}$.

The dangerous modes of this operator are when $K<2$ and $n=m=-s= \pm 1$. For the $K=0$ modes the operator reduces to:

$$
W=-\frac{\partial^{2}}{\partial r^{2}}-\frac{2}{r} \frac{\partial}{\partial r}-\frac{1}{r^{2}}
$$

In ref. [Col81], Coleman presented an elegant way to show that operators which take the above form far away from the origin always have negative eigen ralues due to their attractive centrifugal potential. Consider the following radial function:

$$
\begin{aligned}
\psi & =\frac{1}{r}(\sqrt{r}-\sqrt{R}) \exp (-r / a), \quad r \geq R \\
& =0, \quad r<R
\end{aligned}
$$

where $R$ and $a$ are positive numbers. The expectation value of $W$ from eqn. (2.7.13) for this function is:

$$
\begin{aligned}
<W> & =\int_{0}^{\infty} r^{2} d r \psi(W) \psi \\
& =\int_{0}^{\infty} d r\left[r^{2}(d \psi / d r)^{2}-\psi^{2}\right] \\
& =-\frac{3}{8} \ln (a / R)+\ldots
\end{aligned}
$$

where the triple dots denote terms that have a finite limit as $a \rightarrow \infty$. For any fixed $R$, this expression becomes negative for sufficiently large $a$. To get a negative expectation value for some function, there must be eigenfunctions with negative eigenvalues, since any function can be formed from linear combinations of eigenfunctions. Furthermore, since the proof works for arbitrarily large $R$, no behavior of the fields near the center of the monopole where $F$ and $H$ are nonzero can save $W$ from having negative eigenvalues.

Another way to see that monopole configurations like the one suggested above would not be stable is to see that, unlike the normal 't Hooft-Polyakov monopole 
with a Higg's, these monopoles are not protected by topology at infinity. As $r \rightarrow \infty$, the $A_{0}$ field approaches a constant value of $\eta=2 \pi T / g$ which is simply a temporal gauge transformation of $A_{0}=0$. If $A_{0} \rightarrow 0$, it doesn't matter whether it looks like a hedgehog or is in a uniform color direction, and consequently topology is lost. There is nothing to stop a configuration which has $A_{0}=0$ at both $r=0$ and $r=\infty$ from reducing $A_{0}$ to 0 at intermediate values of $r$ in order to minimize its action. This will be a general problem with any monopoles in pure gauge Yang-Mills theories: finite energy monopole configurations which minimize the effective action will feature the $A_{0}^{a}$ fields approaching minima of the effective potential as $r \rightarrow \infty$. These minima, however, will be gauge equivalent to $A_{0}^{a}=0$, so the monopole configuration will not be stable.

\subsubsection{Generalization to SU(3) with fermions}

To better illustrate these points, I will consider SU(3). From Appendix B, we know that we only need consider field configurations in which $g A_{0} /(2 \pi T)=\nu \lambda_{3}+\sqrt{3} \rho \lambda_{8}$, where $\lambda_{a}$ are the Gell-Mann matrices. Again using Appendix B, we have plotted the effective potential as a function of $\rho$ for $\nu=0$ in fig. 2.7.1. The only minima of the potential in this direction occur at the points $\rho=2 n / 3$ which are just gauge transformations of $\rho=0$ (see Appendix C). Now let us look in the $\lambda_{3}$ direction by setting $\rho=0$ and plotting $V_{\text {eff }}$ as a function of $\nu$ (fig. 2.7.1). The absolute minima are again gauge transformations of $\nu=0$, but in addition there appear to be local minima at $\nu=2 n+1$. By making a contour plot with both $\nu$ and $\rho$ (fig. 2.7.2), however, we can see that the apparent "local minimum" at $\nu=1$ is actually just

the side of a crater which falls to an absolute minimum at $(\nu, \rho)=(1,1 / 3)$. The $A_{0}$ matrix at this minimum has the same eigenvalues as the minimum at $\nu=0$ and $\rho=-2 / 3$, so we know that it is also a gauge transformation of $A_{0}=0$.

On the other hand, true local minima of the effective potential can be created by introducing fermions into the theory and thereby breaking the center symmetry of the 
gauge group (see Appendix C). For example, if one massless fermion is introduced into $\mathrm{SU}(2)$, the absolute minimum at $g\left|A_{0}\right|=2 \pi T$ is transformed into a local minimum (fig. 2.3.1) [Ign92]. Since there is no longer an allowed gauge transformation which takes this minimum to the $A_{0}=0$ configuration, one might be tempted to believe that a stable monopole configuration would exist with the $\left|A_{0}\right| \rightarrow 2 \pi T / g$ as $r \rightarrow \infty$. Unfortunately, the presence of fermions induces no change in the gauge operator $W$, so there are still negative eigenmodes and the monopole is still unstable. It is interesting to note that if a minimum of the effective potential with fermions had occured at any point other than one which was an absolute minimum of the pure gauge theory, then it would have been possible to create a stable monopole configuration which minimized the effective action.

\section{SU(3) Effective Potential}

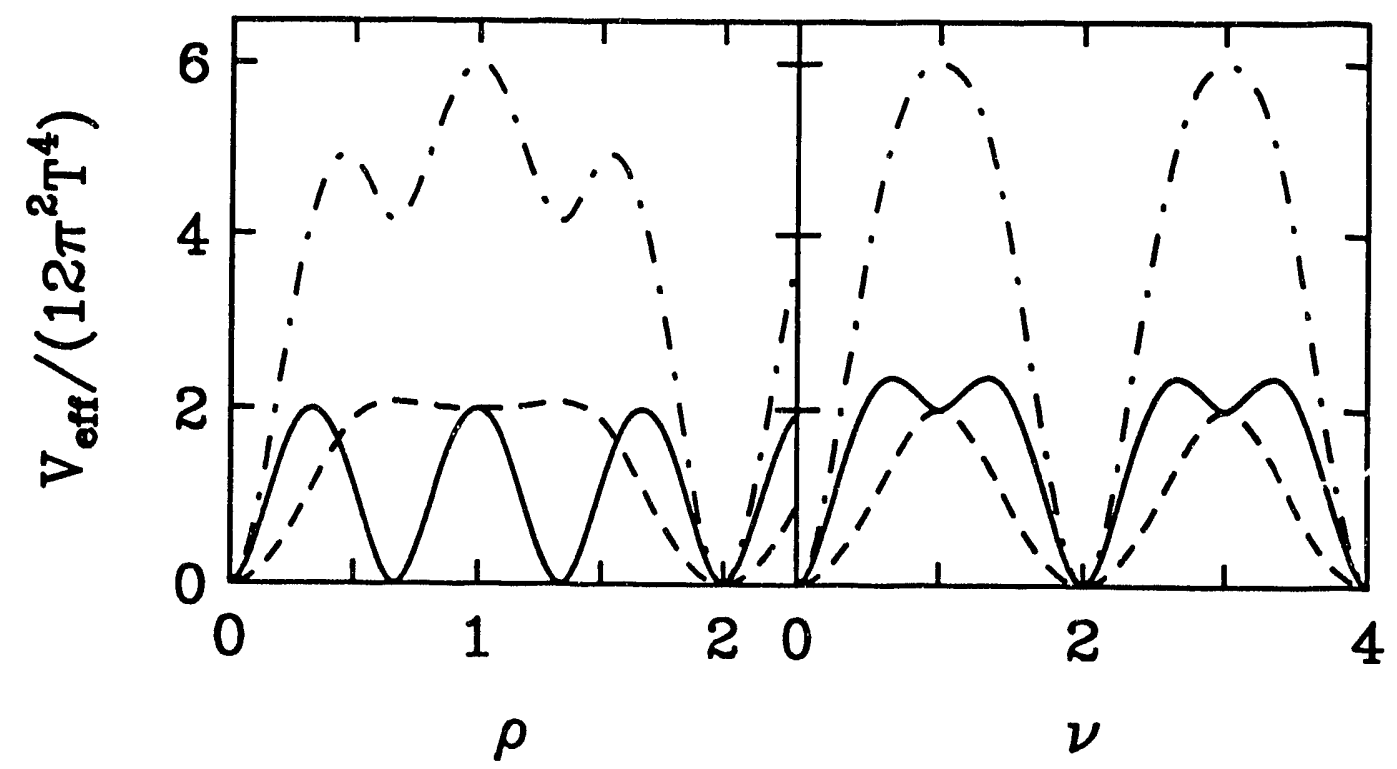

Figure 2.7.1: The one loop SU(3) effective potential for a constant $A_{0}$ field with no fermions (solid), 2 fermions (dot-dashed), and fermions only (dashed). The left frame is for $g A_{0} /(2 \pi T)=\sqrt{3} \rho \lambda_{8}$, while the right frame is for $g A_{0} /(2 \pi T)=\nu \lambda_{3}$. 


\section{Pure Gauge SU(3)}

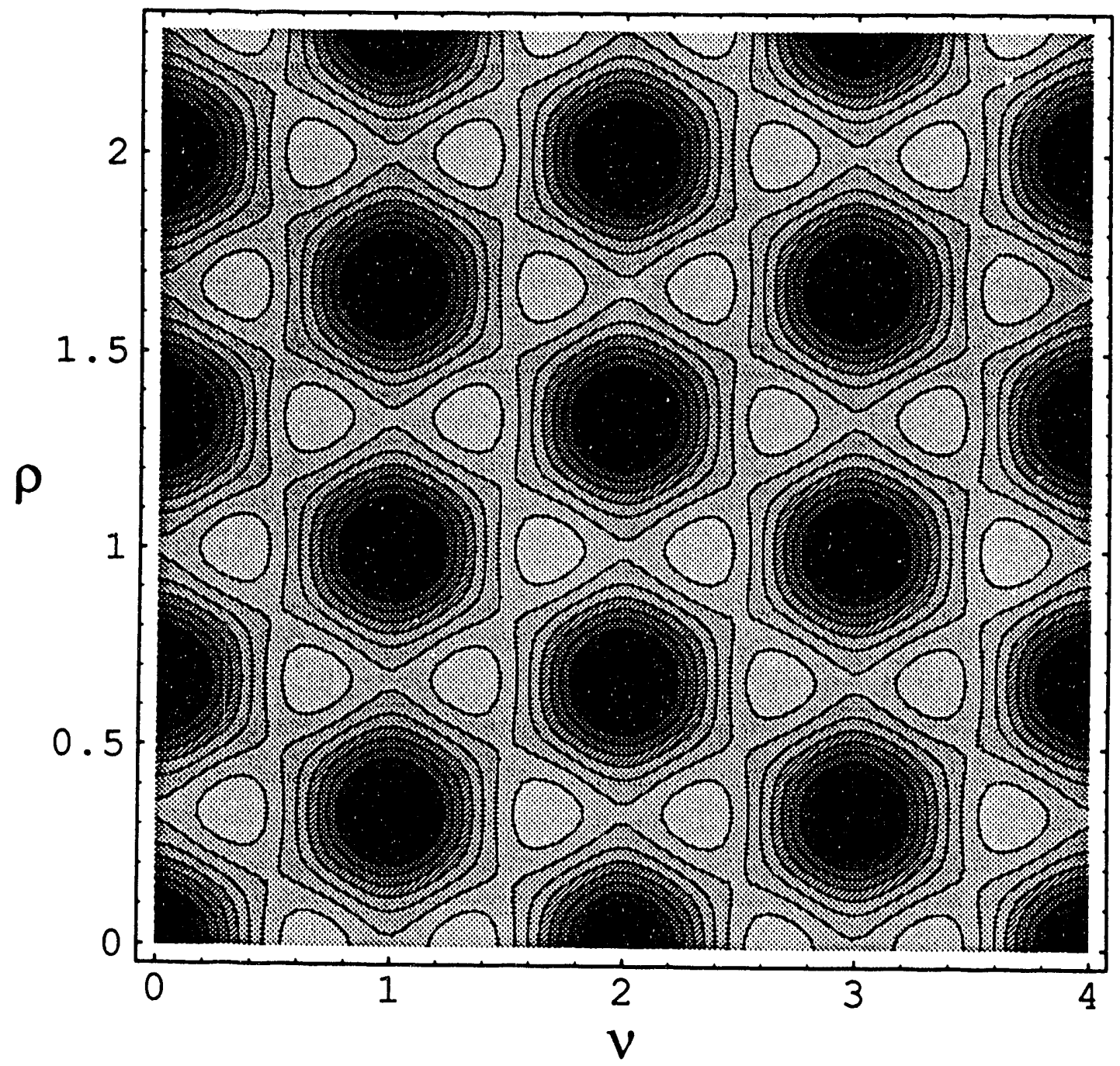

Figure 2.7.2: A contour plot of the one loop pure gauge $\mathrm{SU}(3)$ effective potential for a constant field given by $g A_{0} /(2 \pi T)=\nu \lambda_{3}+\sqrt{3} \rho \lambda_{8}$.

Now we will examine the effect that fermions have on the SU(3) effective potential. Figure 2.7.1 shows this potential as a function of $\rho$ for $\nu=0$. It is not immediately obvious by looking at the figure that the local minima with fermions will be positioned at exactly $2 n \pi T / 3$. Nevertheless, this is the case since the absolute minima of the gauge part of $V_{\text {eff }}$ precisely line up with maxima of the fermionic part (Appendix B). 


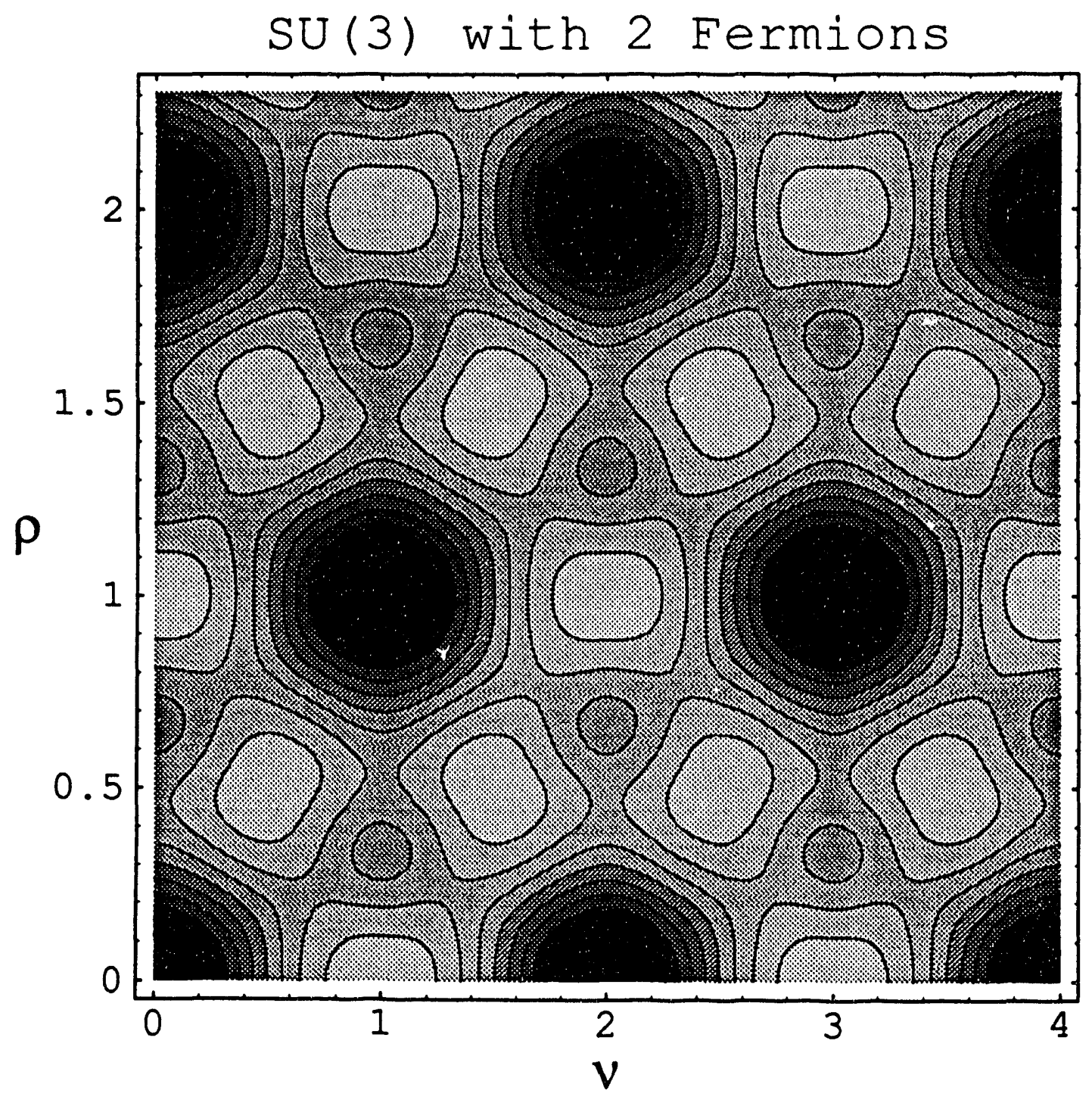

Figure 2.7.3: The SU(3) effective potential as in figure 2.7.2, but with 2 massless fermions.

Similarly, each of the local minima of $(\nu, \rho)$ shown in fig. 2.7 .3 corresponds exactly to an absolute minimum of the pure gauge theory. By the same reasoning used for $\mathrm{SU}(2)$ then, any monopole configuration with $A_{0}$ approaching one of these local minima is still unstable. 


\subsection{Conclusion}

We have developed a covariant derivative expansion of the one loop SU(N) effective action at finite temperature. The main use of this expansion is that it self-consistently produces an infrared cutoff mass which can be used to determine the density of a plasma of solitons in the semiclassical approximation. We have used our technique to evaluate suggestions in the literature[Dah85, Polo91] that magnetically charged solutions to pure gauge $\mathrm{SU}(\mathrm{N})$ could self-stabilize at finite temperature, providing a nonperturbative mechanism for screening static magnetic fluctuations. We have found that classical dyon solutions have infinite energy at the one loop level unless they form an overlapping plasma, in which case they may be difficult to differentiate from thermal fluctuations. In addition, we have found finite energy monopole configurations in $\mathrm{SU}(2)$ and $\mathrm{SU}(3)$ which minimize the effective action but which are unstable. Therefore, at least these two types of semi-classical magnetic configurations do not solve the magnetic screening problem in hot QCD. Nevertheless, if stable, localized, finite energy solutions to pure gauge $\mathrm{SU}(\mathrm{N})$ at $T \neq 0$ are found in the future, then the methods developed here should be useful for estimating their density at high temperatures. 


\section{A Integrals, Sums and Functionals}

In this appendix, we present some of the tools that were used in deriving expressions for the effective action. In order to derive eqns. (2.3.6), (2.3.7), (2.3.9) and (2.3.13), it is necessary to use the following integrals and sums:

$$
\begin{gathered}
\int \frac{d^{3} p}{(2 \pi)^{3}} \exp \left(-p_{i}^{2} t\right)=\frac{1}{(4 \pi t)^{3 / 2}} \\
\int \frac{d^{3} p}{(2 \pi)^{3}} \exp \left(-p_{i}^{2} t\right) p_{j} p_{k}=\frac{1}{2 t(4 \pi t)^{3 / 2}} \delta_{j k} \\
\int \frac{d^{3} p}{(2 \pi)^{3}} \exp \left(-p_{i}^{2} t\right) p_{j} p_{k} p_{l} p_{m}=\frac{1}{(2 t)^{2}(4 \pi t)^{3 / 2}}\left(\delta_{j k} \delta_{l m}+\delta_{j l} \delta_{k m}+\delta_{j m} \delta_{k l}\right) \\
T \sum_{n=-\infty}^{\infty} \exp \left(-p_{0}^{2} t\right)=\frac{1}{2 \sqrt{\pi t}} \sum_{n=-\infty}^{\infty} \exp \left(-\frac{n^{2}}{4 T^{2} t}\right) \\
T \sum_{n} p_{0}^{2} t \exp \left(-p_{0}^{2} t\right)=\frac{1}{2 \sqrt{\pi t}} \sum_{n}\left(\frac{1}{2}-\frac{n^{2}}{4 T^{2} t}\right) \exp \left(-\frac{n^{2}}{4 T^{2} t}\right) \\
T \sum_{n} p_{0}^{4} t^{2} \exp \left(-p_{0}^{2} t\right)=\frac{1}{2 \sqrt{\pi t}} \sum_{n}\left[\frac{3}{4}-3 \frac{n^{2}}{4 T^{2} t}+\left(\frac{n^{2}}{4 T^{2} t}\right)^{2}\right] \exp \left(-\frac{n^{2}}{4 T^{2} t}\right) .
\end{gathered}
$$

The above sums were obtained by using the Poisson summation formula:

$$
\sqrt{\beta} \sum_{n=-\infty}^{\infty} F(n \beta)=\sqrt{\alpha} \sum_{r=-\infty}^{\infty} f(n \alpha)
$$

where $\alpha \beta=2 \pi$ and $F(x)$ and $f(p)$ are Fourier transforms of each other. The sums on the left sides of (A.4-A.6) are over Matsubara frequencies, while those on the right side are over $T=0(n=0)$ and $T \neq 0(n \neq 0)$ pieces. The latter can be seen by noticing that in the limit as $T \rightarrow 0$, only the $n=0$ terms survive.

In addition, we define the following functionals for notational convenience:

$$
H_{n}=\operatorname{tr} \int d^{4} x D_{0}^{2 n}
$$




$$
\begin{aligned}
H_{1} & =-g^{2} N \int d^{4} x A_{0}^{2} \\
F_{2} & =\operatorname{tr} \int d^{4} x\left[D_{\mu}, D_{\nu}\right]^{2}=-g^{2} N \int d^{4} x\left(F_{\mu \nu}^{a}\right)^{2} \\
D_{2} & =\operatorname{tr} \int d^{4} x\left[D_{j}, D_{0}\right]^{2}=-g^{2} N \int d^{4} x\left(F_{j 0}^{a}\right)^{2} \\
G_{n+1} & =\operatorname{tr} \int d^{4} x\left[D_{j},\left[D_{j}, D_{0}^{2 n}\right]\right] \\
G_{n+1}^{\prime} & =-2 T^{2} \operatorname{tr} \int d^{4} x\left[D_{i},\left[D_{i}, \cos \left(n D_{0} / T\right)\right]\right]
\end{aligned}
$$

where the last definition was introduced in eqn. (2.5.4) while developing the improved expansion. For a constant $A_{0}^{a}$ field, all of the above functionals vanish except

$$
H_{n}=\operatorname{tr} \int d^{4} x\left(g A_{0}\right)^{2 n},
$$

where we use the matrix notation $A_{0}=f^{a b c} A_{0}^{c}$. For SU(2) with $\left|A_{0}\right|=\eta$, we get the simple form:

$$
H_{n}=2\left(-g^{2} \eta^{2}\right)^{n} V / T
$$

The following high temperature approximations were used in deriving eqns. (2.3.10), (2.3.11) and (2.3.13):

$$
\begin{aligned}
\sum_{n=1}^{\infty} n^{2 p} \exp \left(-\epsilon n^{2}\right) & \simeq \frac{1}{2} \sqrt{\pi / \epsilon} \frac{(2 p-1) ! !}{(2 \epsilon)^{p}}-\frac{1}{2} \delta_{p 0} \\
\sum_{n=1}^{\infty} \frac{1}{n^{2}} \exp \left(-\epsilon n^{2}\right) & \simeq \frac{1}{2} \epsilon-\sqrt{\pi \epsilon}+\frac{1}{6} \pi^{2} \\
\sum_{n=1}^{\infty} \operatorname{Ei}\left(-\epsilon n^{2}\right) & \simeq-\sqrt{\pi / \epsilon}-\frac{1}{2} \ln \epsilon+1.55 .
\end{aligned}
$$

The above sums become exact in the limit as $\epsilon \rightarrow 0$ and are even good to within a few percent when $\epsilon=1$. We also used the following integral to regulate the ultraviolet divergences in (2.3.11) and (z.3.13):

$$
\int_{0}^{\delta} \frac{d t}{t}\left(1-e^{-\Lambda^{2} t}\right)=\gamma_{E}+\ln \left(\delta \Lambda^{2}\right),
$$

where $\gamma_{E} \simeq 0.577$ is Euler's constant. 


\section{B Effective Potentials for $T \neq 0$ SU(N)}

The $A_{0}$ fields in $\mathrm{SU}(\mathrm{N})$ can always be expressed in terms of a traceless Hermitian $N \times N$ matrix by defining $A_{0}=\frac{1}{2} A_{0}^{a} \lambda_{a}$ where $\frac{1}{2} \lambda_{a}$ are the $N^{2}-1$ fundamental generators of $\mathrm{SU}(\mathrm{N})$. Any matrix of this form can be diagonalized by a unitary transformation. However, making such a unitary transformation is equivalent to making a time-independent gauge transformation on $A_{0}$. Since the effective potential must be invariant under all gauge transformations, it can only depend on the eigenvalues of $A_{0}$, so it is sufficient to study configurations in which $A_{0}$ is diagonal. In Appendix D of ref.[Gro81], Gross et al. evaluate functioñai determinants for constant fields which are diagonal in color. We use thein results to write down a general expression for the effective potential of any traceless, diagonal $A_{0}$ rnatrix. Let

$$
g A_{0}=2 \pi T q
$$

where $\mathrm{q}$ is a diagonal, real and traceless matrix whose elements are given by

$$
(q)_{j k}=q^{j} \delta_{j k}
$$

The effective potential for this field configuration is given by[Gro81]:

$$
V_{\text {eff }}=\frac{2 T^{4}}{\pi^{2}} \sum_{n=1}^{\infty} \sum_{j=1}^{N}\left\{2 N_{f}(-1)^{n} \frac{\cos \left(n \pi q^{j}\right)}{n^{4}}-\sum_{k=1}^{N} \frac{\cos \left(n \pi\left(q^{j}-q^{k}\right)\right)}{n^{4}}\right\}+\frac{\pi^{2} T^{4}}{45} .
$$

The sums over $n$ can be done by using the following relations:

$$
\begin{gathered}
\sum_{n=1}^{\infty} \frac{\cos (n \pi x)}{n^{4}}=\frac{\pi^{4}}{90}-\frac{\pi^{4}}{48}[x]_{+}^{2}\left([x]_{+}-2\right)^{2} \\
\sum_{n=1}^{\infty}(-1)^{n} \frac{\cos (n \pi x)}{n^{4}}=-\frac{7 \pi^{4}}{720}+\frac{\pi^{4}}{48}\left(2[x]_{-}^{2}-[x]_{-}^{4}\right),
\end{gathered}
$$

where $[x]_{+}=[x(\bmod 2)]$ and $[x]_{-}=[(x+1) \bmod 2]-1$.

For $\mathrm{SU}(2)$, there is only one possible form of traceless diagonal matrix: $q=\nu \sigma_{3}$. The effective potential then takes the form:

$$
V_{\mathrm{eff}}=\frac{\pi^{2} T^{4}}{12}\left\{2 N_{f}\left(2[\nu]_{-}^{2}-[\nu]_{-}^{4}\right)+[2 \nu]_{+}^{2}\left([2 \nu]_{+}-2\right)^{2}\right\}-\frac{\pi^{2} T^{4}}{15}\left(1+\frac{7 N_{f}}{6}\right) .
$$


Dropping the constant term at the end, $V_{\text {eff }}$ for $S U(2)$ is plotted in fig. 2.3.1. For $\mathrm{SU}(3)$, there are two diagonal generators, so an arbitrary diagonal $\mathrm{SU}(3)$ matrix can be expressed by $q=\nu \lambda_{3}+\sqrt{3} \rho \lambda_{8}$. The effective potential then takes the form:

$$
\begin{aligned}
V_{\mathrm{eff}} & =\frac{\pi^{2} T^{4}}{12}\left\{N_{f}\left(2[\nu+\rho]_{-}^{2}-[\nu+\rho]_{-}^{4}+2[\nu-\rho]_{-}^{2}-[\nu-\rho]_{-}^{4}+2[2 \rho]_{-}^{2}-[2 \rho]_{-}^{4}\right)\right. \\
& \left.+[2 \nu]_{+}^{2}\left([2 \nu]_{+}-2\right)^{2}+[\nu+3 \rho]_{+}^{2}\left([\nu+3 \rho]_{+}-2\right)^{2}+[\nu-3 \rho]_{+}^{2}\left([\nu-3 \rho]_{+}-2\right)^{2}\right\} \\
& -\frac{\pi^{2} T^{4}}{45}\left(8+\frac{21 N_{f}}{4}\right) .
\end{aligned}
$$

$V_{\text {eff }}$ for $\mathrm{SU}(3)$ is plotted in figs. 2.7.1 - 2.7.3.

There are more allowed gauge transformations in pure gauge $\mathrm{SU}(\mathrm{N})$ than there are in $\mathrm{SU}(\mathrm{N})$ with fermions (see Appendix C). For this reason, some of the degenerate absolute minima of the pure gauge effective potential are no longer absolute minima when fermions are included in the theory. Nevertheless, we show here that these ir ts remain stationary points of the complete effective potential with fermions. A general diagonal $\mathrm{SU}(\mathrm{N})$ matrix can always be written as a linear combination of matrices having at least one zero on the diagonal and the matrix $\lambda_{N^{2}-1}$ given by:

$$
\lambda_{N^{2}-1}=\operatorname{diag}(1,1, \ldots, 1-N) \nu
$$

Only this last matrix will feature the minima we seek (see Appendix C), so we only need to consider its effective potential:

$$
\begin{aligned}
V_{\mathrm{eff}} & =\frac{2 T^{4}}{\pi^{2}} \sum_{n=1}^{\infty} \frac{1}{n^{4}}\left\{2 N_{f}(-1)^{n}[(N-1) \cos (n \pi \nu)+\cos (n(N-1) \pi \nu)]\right. \\
& \left.-2(N-1) \cos (n N \pi \nu)-(N-1)^{2}\right\} .
\end{aligned}
$$

By simple differentiation, it is easy to verify that the minima at $\nu=2 \mathrm{~m} / N$ of the pure gauge part correspond exactly to maxima of the fermionic part. Consequently, for any value of $N_{f}$, the full effective potential will always have stationary points at $\nu=2 m / N$. 


\section{Allowed Gauge Transformations}

Since there are periodic temporal boundary conditions for the fields at finite temperature, the only allowed gauge transformations are those which preserve the boundary conditions. We would like to determine the most general form of these allowed gauge transformations. A general unitary transformation can always be written as an exponential:

$$
U=\exp \left[i \lambda_{a} \theta^{a}(\tau, \vec{x})\right],
$$

where $\frac{1}{2} \lambda_{a}$ are the generators of the group. Let us now perform a gauge transformation on $A_{0}=\frac{1}{2} \lambda_{a} A_{0}^{a}$ :

$$
A_{0} \rightarrow A_{0}^{\prime}=U A_{0}^{T^{\top-1}}-\frac{i}{g}\left[\partial_{0} U\right] U^{-1} .
$$

As usual, the first term simply rotates $A_{0}^{a}$ in color space, while the second term changes its magnitude. Just looking at the second term, we can see that the magnitude of $A_{0}^{\prime}(\tau)$ will only be the same as that of $A_{0}^{\prime}(\tau+\beta)$ if $\theta^{a}(\tau, \vec{x})$ takes the form:

$$
\theta^{a}(\tau, \vec{x})=f_{1}^{a}(\tau, \vec{x})+f_{2}^{a}(\vec{x}) \tau,
$$

where $f_{1}^{a}(\tau+\beta, \vec{x})=f_{1}^{a}(\tau, \vec{x})$. Now if we examine the first term of eqn. (C.2), we can see that periodicity for a pure gauge theory also implies:

$$
U(\tau+\beta, \vec{x})=\exp (i \alpha) U(\tau, \vec{x}) .
$$

This puts a restriction on $f_{2}^{a}$. For $\mathrm{SU}(\mathrm{N})$, the only possible values of $\exp (i \alpha)$ will be $N$ 'th roots of 1 , which form the center of the group. These discrete allowed values of $\exp (i \alpha)$ can only be generated by discrete values of $\theta^{a}$. Together with eqn. (C.4), this implies that there can be no $\vec{x}$ dependence for $f_{2}$, since such a dependence would be continuous rather than discrete.

For SU(2),

$$
U=\cos \left(\left|\theta^{a}\right|\right)+i \lambda_{a} \hat{\theta}^{a} \sin \left(\left|\theta^{a}\right|\right),
$$

where $\hat{\theta^{a}}=\theta^{a} /\left|\theta^{a}\right|$, and it is easy to see that only $f_{2}^{a}=n_{a} \pi T$ with integer $n_{a}$ will satisfy eqn. (C.4). For $\mathrm{SU}(\mathrm{N})$ with $N>2$, it is always possible to choose a fundamental 
representation in which all but one of the generators have at least one zero eigenvalue (for example the Gell-Mann matrices for $\mathrm{SU}(3)$ ). The $f_{2}^{a}$ terms corresponding to each of the generators with a zero eigenvalue must be of the form $f_{2}^{a}=2 n_{a} \pi$. The remaining generator $\lambda_{N^{2}-1}$ is given in its unnormalized form by (B.8) in Appendix B. It can be verifon that $f_{2}^{N^{2}-1}=2 n \pi / N$ gives rise to allowed gauge transformations with $\exp (i \alpha)$ of (C.4) equal to $N$ 'th roots of 1 .

The situation changes a bit if there are fermions in the theory. Since fermions transform like $\psi \rightarrow U \psi$, there are no factors of $U^{-1}$ to cancel global phases. Thus in order for fermion tempora boundary conditions to remain unaffected by gauge transformations, only transformations satisfying eqn. (C.4) with $\alpha=0$ are permissible. In other words, fermions break the center symmetry which is present in pure gauge theories. Therefore, the most general form of $f_{2}^{a}$ for $\mathrm{SU}(\mathrm{N})$ with fermions is $f_{2}^{a}=2 n \pi T$. 


\section{Bibliography}

\section{Introduction}

[Christ] N.H. Christ, Nucl. Phys. A544, 81 (1992).

[Fuku] M. Fukugita et al., Phys. Rev. C68, 761 (1992).

[LLew] C.H. Llewellyn Smith, Phil. Trans. R. Soc. Lond. A304, 5 (1982).

\section{Chapter 1}

[Ame91] N.S. Amelin et al., Phys. Rev. C44, 1541 (1991).

[Bloo] M.A. Bloomer, MIT Ph.D thesis (1990); private communication.

[Brown] G. Brown et al., Phys. Rev. C43, 1881 (1991);

C.M. Ko et al., Phys. Rev. Lett. 66, 2577 (1991).

[BrownEr] C.M. Ko et al., Erratum Phys. Rev. Lett. 67, 1811 (1991).

[Chap91] S. Chapman and M. Gyulassy, Phys. Rev. Lett. 67, 1210 (1991).

[Chap92] S. Chapman and M. Gyulassy, Phys. Rev. C45, 2952 (1992).

[Clare] R.B. Clare and D. Strottman, Phys. Rep. 141, 177 (1986).

[Dan] P. Danielewicz and J.M. Namyslowski, Acta Phys. Pol. B12, 695 (1981).

[Das] S. Das Gupta and A.Z. Mekjian, Phys. Rep. 72, 131 ( 1981).

[E802] T. Abbott et al. (E802 collab), Phys. Rev. Lett. 64, 847 (1990); Phys. Rev. Lett. 66, 1567 (1991).

[E802a] T. Abbott et al. (E802 collab), 2. Phys. C38, 135 (1988). 
[E810] W.A. Love et al. (E810 collab), Nucl. Phys. A525, 601c (1991).

[E814] J. Barrette et al. (E814 collab), Phys. Rev. Lett. 64, 1219 (1990);

Nucl. Phys. A544, 137 (1992).

[E814a] W.E. Cleland et al., Nucl. Phys. A525, 91c (1991).

[E866] M. Gonin et al. (E866 collab), Presented at the International Nuclear Physics Conference, Wiesbaden, Germany, Brookhaven Report BNL-47925 (1992).

[Fox] D. Fox, private communication.

[Gosset] J. Gosset, J. Kapusta, G.D. Westfall, Phys. Rev. C18, 844 (1978).

[Gyu87] M. Gyulassy, in Proceedings of the Balatonfured Conference on Nuclear Physics, CERN Report, CERN-TH.4794 (1987).

[Gyu90] M. Gyulassy, in Proceedings of the Workshop on Heavy Ion Physics at the AGS, Brookhaven Report, BNL-44911 (1990), LBL Report LBL-29076 (1990).

[HIPAGS]F. Videbaek, in Procus of the Workshop on Heavy Ion Physics at the AGS, Brookhaven Report, BNL-44911 (1990), LBL Report LBL-29076 (1990); private communication.

[Lund] B. Andersson, et. al., Nucl. Phys. B281, 289 (1987).

[Mish] I.N. Mishustin et al., Nucl. Phys. A494, 595 (1989).

[Myers] W.D. Myers, Nucl. Phys. A296, 177 (1978).

[Nag] S. Nagamiya, Nucl. Phys. A544, 5c (1992).

[pA] W. Busza and A. Goldhaber, Phys. Lett. 139B, 235 (1984);

W. Busza and R. Ledoux, Ann. Rev. Nucl. Part. Sci. 38, 119 (1988);

S.Date, M. Gyulassy, and H. Sumiyoshi, Phys. Rev D32, 619 (1985).

[PANIC] T. Abbott et al. (E802 collab), Phys. Lett. 197B, 285 (1987);

M. S. Tannenbaum et al., Nucl. Phys. A488, 555c (1988);

P. Braun-Munzinger et al., Z. Phys. C38, 45 (1988);

J. Stachel, Nucl. Phys. A527, 167c (1991). 
[Pars] C. Parsons, MIT Ph.D. Thesis (1992).

[QM88] Proceedings of Quark Matter '88, ed. by G. Baym,

P. Braun-Munzinger, S. Nagamiya, Nucl. Phys. A498, 1c (1989).

[RQMD80?] F. Videbaek, in Proceedings of the International Symposium on High Energy Nuclear Collisions and Quark Gluon Plasma, Kyoto, Japan, Brookhaven Report, BNL-46546 (1991).

[RQMD91] H. Sorge, H. Stöcker, W. Greiner, Ann. Phys. 192, 266 (1989);

H. Sorge et al., Nucl. Phys. A525, 95c (1991);

R. Mattiello et al., Phys. Rev. Lett. 63, 1459 (1989).

[RQMD92] H. Sorge et al., Phys. Rev. Lett. 68, 286 (1992).

[RQMDpi] H. Sorge et al., UFTP preprint 263 (1991).

[Stach] J. Stachel, and P. Braun-Munzinger, Phys. Lett. 216B, 1 (1989);

Nucl. Phys. A498, 577c (1989); private communication.

[Stau89] E. F. Staubo et al., Phys. Lett. 229B, 351 (1989).

[Stau90] E. F. Staubo et al., Physica Scipta T32, 190 (1990).

[Strot] Dan Strottman, private communication.

[Vide] F. Videbaek, E802 Internal Memo E-802-MEM-56 (unpublished); private communication.

\section{Chapter 2}

[Act79] A. Actor, Rev. Mod. Phys. 51, 4 (1979).

[Ani84] R. Anishetty, J. Phys. G10, 439 (1984).

[Bel91] V. M. Belyaev, Phys. Lett. 241B, 91 (1990);

Phys. Lett. 254B, 153 (1991).

[Bia76] F.A. Bias and J.R. Primack, Phys. Rev. D13, 819 (1976).

[Bra90] E. Braaten and R. Pisarski, Nucl. Phys. B337, 569 (1990). 
[Chan86] L.-H. Chan, Phys. Rev. Lett. 54, 1222 (1985);

Phys. Rev. Lett. 55, 21 (1985);

Phys. Rev. Lett. 57, 1199 (1986).

[Che87] O. Cheyette UC Berkeley Ph.D. Thesis, LBL-23247-mc (1987);

Phys. Rev. Lett. 55, 2394 (1985);

Nucl. Phys. B297, 183 (1988);

O. Cheyette and M. Gaillard Phys. Lett. 197B, 205 (1987).

[Col73] S. Coleman and E. Weinberg, Phys. Rev. D7, 1888 (1973).

[Col77] S. Coleman, "The Uses of Instantons" (1977), in Aspects of Symmetry, Cambridge University Press, Cambridge (1985).

[Col81] S. Coleman, "The Magnetic Monopole Fifty Years Later", in Erice Subnuclear Lectures The Unity of the Fundamental Interactions edited by Zichichi, (1981).

[Cor81] E. Corrigan and P. Goddard, Comm. Math. Phys. 80, 575 (1981).

[Dah85] K. J. Dahlem, Z. Phys. C29, 553 (1985).

[deW67] B.S. deWitt, Phys. Rev. 162, 1195, 1239 (1967).

[Dya84] D'yakonov et. al. Sov. J. Nucl. Phys. 39, 1 (1984).

[Fie89] R. D. Field, Applications of Perturbative QCD, Addison-Wesley, New 'Tork (1989).

[Gai89] M. K. Gaillard, Nucl. Phys. B268, 669 (1986);

J. Burton et al., Phys. Rev. D41, 3118 (1989).

[Gro81] D. Gross et al., Rev. Mod. Phys. 53, 43 (1981).

[Ign92] J. Ignatius et. al. Phys. Rev. Lett. 68737 (1992).

[Jon81] T. Jonsson, Lett. Math. Phys. 5, 81 (1981).

[Kap79] J. I. Kapusta, Nucl. Phys. B148 461 (1979);

Finite-Temperature Field Theory,

Cambridge University Press, New York (1989).

[Lin80] A. D. Linde, Phys. Lett. 96B, 289 (1980). 
[Man76] S. Mandelstam, Phys. Rep. 23C, 245 (1976);

Phys. Rep. 67, 109 (1980);

"The Possible Role of Monopoles in the Confinement Mechanism", in Monopoles in Quantum Field Theory (Proceedings of the Monopole Meeting in Trieste, Italy, December 1981), edited by N.S. Craigie et. al., World Scientific Publishing, Singapore (1982).

[Min86] H. Min, Hamburg Desy preprint DESY 86-152 (1986);

P. Binetruy and M. Gaillard, Phys. Rev. D32, 931 (1985).

[Mor79] P.D. Morley and M.B. Kislinger, Phys. Rep. C51, 63 (1979).

[Mot78] E. Mottola, Phys. Lett. 79B, 242 (1978).

[Mul85] B. Müller, The Physics of the Quark Gluon Plasma, Springer Verlag, New York (1985).

[Ole81] P. Oleson, "Confinement and Magnetic Condensation for $N \rightarrow \infty$ ", in Monopoles in Quantum Field Theory (Proceedings of the Monopole Meeting in Trieste, Italy, December 1981), edited by N.S. Craigie et. al., World Scientific Publishing, Singapore (1982).

[Polo91] J. Polonyi, Phys. Lett. 240B, 183 (1990);

M. Oleszczuk and J. Polonyi, MIT preprint MIT-CTP-1984 (1991).

[Polo92] J. Polonyi, private communication.

[Poly75] A.M. Polyakov, Sov. Phys.-JETP 41, 988 (1975);

Phys. Lett. 59B, 82 (1975).

[Poly77] A.M. Polyakov, Nucl. Phys. B120, 429 (1977).

[Pra75] M.K. Prasad and C.M. Sommerfield, Phys. Rev. Lett. 35, 760 (1975).

[Raj82] R. Rajaraman, Solitons and Instantons, North Holland Publishing Company, New York (1982).

[Sav77] G.K. Savvidy, Phys. Lett. 71B, 133 (1977).

[Sla80] L.D. Faddeev and A.A. Slavnov, Gauge Fields: Introduction to Quantum Theory, Benjamin/Cummings, Reading Mass. (1980).

[tHo74] G. 't Hooft, Nucl. Phys. B79, 276 (1974). 
[tHo76] G. 't Hooft, Phys. Rev. D14, 3432 (1976).

[War81] R.S. Ward, Phys. Lett. 102B, 136 (1981).

[Wei81] N. Weiss, Phys. Rev. D24, 475 (1981).

[Wu68] T.T. Wu and C.N. Yang, in Properties of Matter Under Unusual Conditions, edited by H. Mark and S. Fernbach, Interscience, New York (1969) p. 349. 

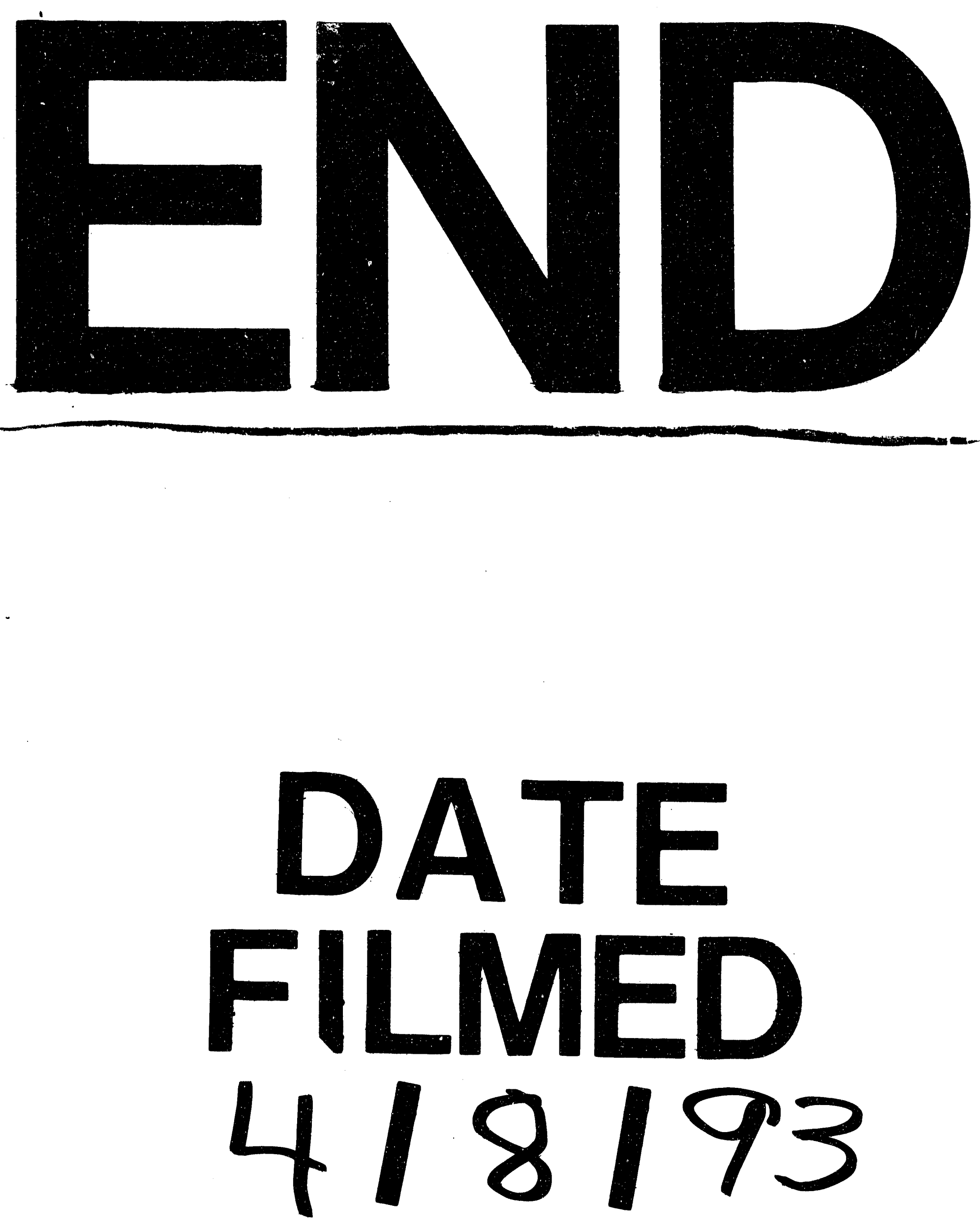
